MODELLING POPULATION DYNAMICS AND

PERSISTENCE

IN FRAGMENTED LANDSCAPES 
Examining committee:

Prof.dr.ir. A. Veldkamp

Prof. Dr. Z. Su

Prof.dr. P. Verburg

Prof.dr. T.P. Dawson
University of Twente, The Netherlands University of Twente, The Netherlands VU University Amsterdam, The Netherlands University of Dundee, UK

ITC dissertation number 253

ITC, P.O. Box 217, 7500 AA Enschede, The Netherlands

ISBN 978-90-365-3750-6

DOI $10.3990 / 1.9789036537506$

Cover designed by Xinping Ye

Printed by ITC Printing Department

Copyright (c) 2014 by Xinping Ye

\section{UNIVERSITY OF TWENTE.}

U1C Faculty of geO-INFORMATION SCIENCE AND EARTH OBSERVATION 


\title{
MODELLING POPULATION DYNAMICS AND PERSISTENCE IN FRAGMENTED LANDSCAPES
}

\author{
DISSERTATION
}

to obtain

the degree of doctor at the University of Twente, on the authority of the rector magnificus, prof.dr. H. Brinksma, on account of the decision of the graduation committee, to be publicly defended

on Thursday October 2, 2014 at 12:45 hrs

by

Xinping Ye

born on 10 January 1976

in Jiangxi, China 
This thesis is approved by

Prof. dr. Andrew K. Skidmore, promoter

Dr. Tiejun Wang, co-promoter 
With no small steps, one can't cover a thousand mile journey; with no small streams, there can be no oceans or seas. 不积跬步, 无以至千里, 不积小流, 无以成江海。

- 《荀子·劝学》 



\section{Acknowledgements}

This work could not have been completed without the input and support of many people. Here I would like to take this opportunity to use simple words to express my deepest gratitude.

Firstly, I am very grateful to my promotor Prof. Dr. Andrew Skidmore for his enlightening guidance and inspiring instruction in the development and completion of this study, and for continually pushing me to become more critical in my scientific thinking. He always had confidence in me to carry out this research, and always gave me freedom to make my own choices. Without his support, none of this can be achieved. I also extend my gratitude to Eva Skidmore, for her excellent English editing service.

I am indebted to my assistant promotor Dr. Teijun Wang and his wife Wen Xue for their generous support and assistance over these years. The deepest thanks go to Tiejun for always being available to discuss my doubts and findings, and sharing his experience of staying in Holland. My greatest gratitude goes to Wen for her encouragement and taking care of me in these years. Without their supports, this thesis would likely not have been accomplished so quickly.

I am appreciative to Dr. Paul van Dijk, Ms. Loes Colenbrander, and all staff and faculty members of the NRS Department for their assistance. Special thanks goes to Ms. Esther Hondebrink for kind helps in Dutch translation.

I am grateful to Dr. Lael Parrott, Dr. Daniel Fortin, Dr. Xuehua Liu, and Dr. Changqing Yu for their generous assistance and invaluable suggestions.

Many thanks also go to my Chinese community for their company and friendship, in particular to Xiaojing $\mathrm{Wu}$, Sudan $\mathrm{Xu}$, and Biao Xiong and other Chinese PhD students for their support and help.

I would like to thank EU Erasmus Mundus Programme and ITC for providing me with a fully funded $\mathrm{PhD}$ position to work on this thesis.

Finally, I feel most deeply indebted to my parents for their great support over the years. A big thanks to my old sisters and old brother, they help me to take care of my parents. I am truly grateful to my wife Hongcai Liu and lovely daughter Yezi for their support and tolerance during my PhD study. I dedicate this dissertation to them. 


\section{Table of Contents}

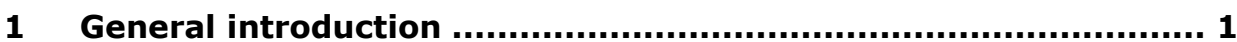

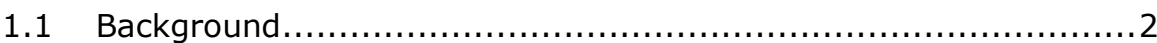

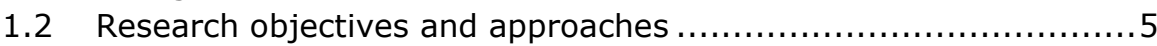

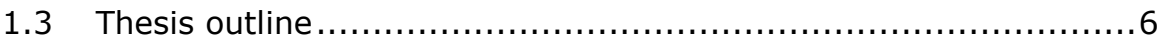

2 Within-patch habitat quality determines the resilience of specialist species in fragmented landscapes ............................... 9

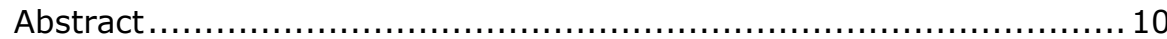

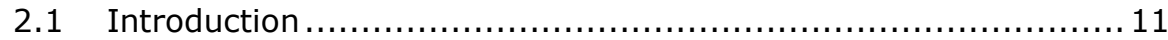

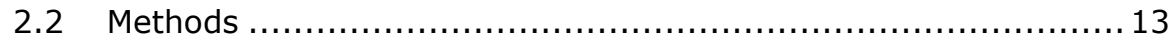

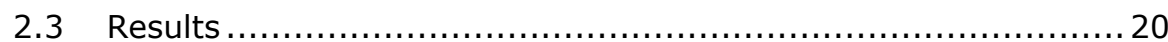

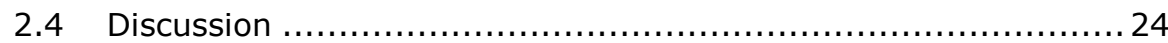

3 Spatial pattern of habitat quality modulates population persistence in fragmented landscapes ................................ 33

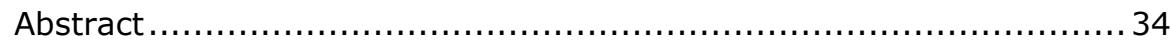

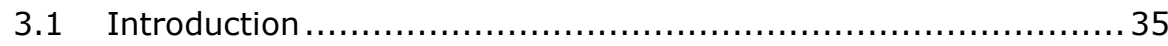

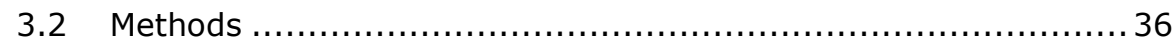

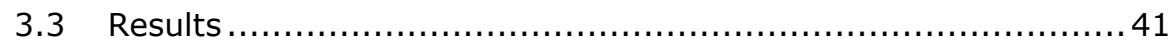

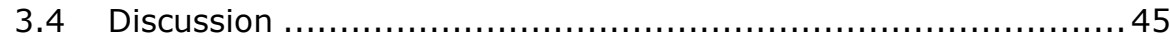

4 Joint effects of habitat heterogeneity and species' lifehistory traits on population dynamics in spatially structured landscapes .......................................................................... 51

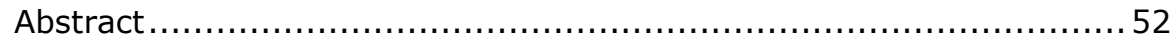

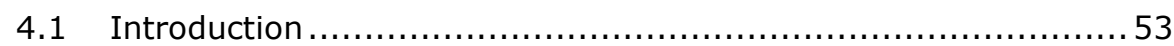

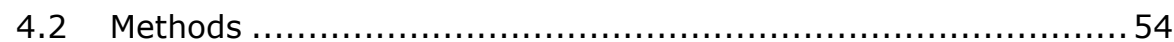

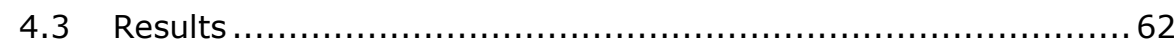

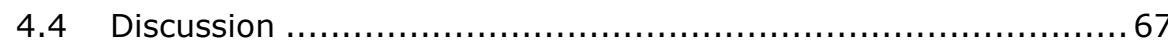

5 A wavelet-based approach to evaluate the roles of structural and functional landscape heterogeneity in animal space use at multiple scales ................................................................ 77

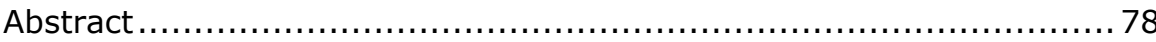

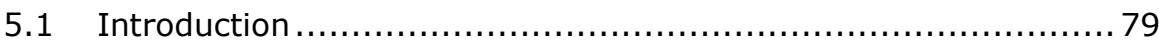

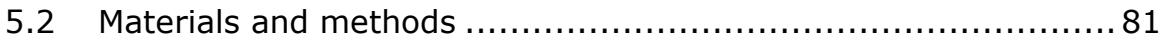

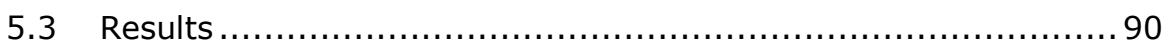

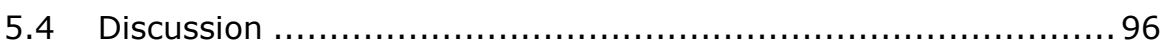

6 General discussion ..........................................................105

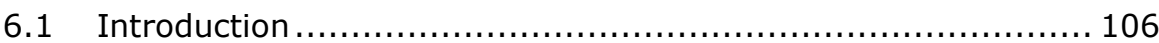

6.2 Relative influences of patch geometry and within-patch heterogeneity on population dynamics........................... 106

6.3 Impacts of spatial pattern of within-patch heterogeneity on patchy populations

6.4 Joint effects of landscape spatial structure and species' lifehistory traits on population dynamics 


\subsection{Differential roles of structural and functional landscape}

heterogeneity in animal space use .................................... 109

6.6 General conclusion.................................................. 111

6.7 Suggestions for future research ................................. 111

Bibliography................................................................................113

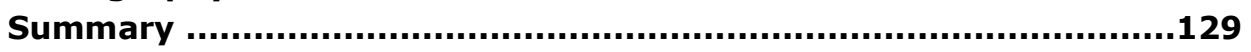

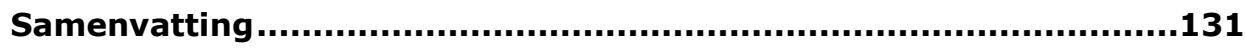

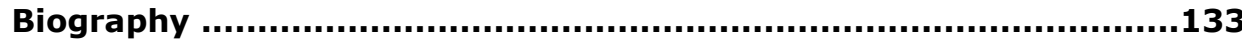

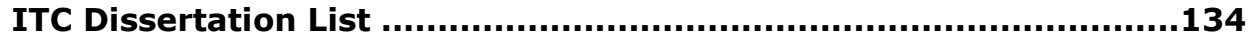


1 General introduction 


\subsection{Background}

Understanding the driving forces that affect population dynamics and persistence in patchy environments is a central concern of ecology (Stacey \& Taper, 1992; Lawler et al., 2006; Melbourne \& Hastings, 2008). Many theoretical and empirical studies suggest that a large area is capable of supporting larger populations than a smaller area, thereby reducing the likelihood of stochastic extinctions (Andrén, 1994; Fahrig, 1997; With \& King, 1999; Flather \& Bevers, 2002). One popular approach for predicting population viability in fragmented landscapes is the "patch area-isolation" paradigm that built upon the theories of island biogeography and metapopulation biology (Hanski \& Gaggiotti, 2004; Prugh et al., 2008). This paradigm assumes the landscape to be a binary system of uniformly "suitable" habitat surrounded by an "inhospitable" matrix, for which the probability of species persistence in landscapes varies as a function of habitat patch size and isolation (Hanski, 1998; Heaney, 2000). However, defining a landscape as discrete patches of habitat and nonhabitat ignores gradients of habitat suitability and differences between species with respect to what constitutes suitable habitat for them (Wiegand et al., 1999; Humphries et al., 2013). Though the patch area-isolation paradigm has been validated for small groups of species (e.g. Hanski et al., 1995; Moilanen et al., 1998), the general ability of patch area and isolation to predict population dynamics in patchy environments remains questionable (Prugh et al., 2008; Schooley \& Branch, 2009). There has been increasing evidence that this paradigm fails to predict the performance of populations in fragmented landscapes (see Walker et al., 2003; Franken \& Hik, 2004; Pellet et al., 2007). For instance, Pellet et al. (2007) analysed multiyear occupancy data of ten species and found that patch area was not a strong predictor of extinction risk due to a weak correlation between patch area and population size, nor did connectivity generally improve predictions of colonization. The area-isolation framework may require incorporation of habitat heterogeneity to improve our understanding of the ecological mechanisms underlying population dynamics in patchy landscapes (Fleishman et al., 2002; Schooley \& Branch, 2007).

Natural environments are typically heterogeneous, wherein populations encounter spatial (and temporal) variation in both abiotic and biotic conditions (Kawecki, 1995). Such variations in the distribution and abundance of vital resources form a mosaic of sites of differing habitat quality within or between habitat patches, or specifically, landscape heterogeneity (Lovett et al., 2005; Shaver, 2005). Many studies have already shown that spatial variation in habitat quality between habitat patches (i.e., patch quality) have strong effects on population density and extinction events (Clarke et al., 1997; Sutcliffe et al., 1997; Thomas et al., 2001; Franzén \& Nilsson, 2010). For instance, Fleishman et al. (2002) found that the quality of 
habitat patches affected occupancy and turnover rates, while Franken and Hik (2004) found patch quality played a major role in colonization and extinction of local populations. These studies, however, focused on interpatch variation in habitat quality (Pulliam, 1988; Runge et al., 2006), and thus contain little information about the influence of spatial (and temporal) heterogeneity within habitat patches. In contrast, the ecological consequence of within-patch heterogeneity has received little attention.

From an ecological standpoint, spatial variation in local habitat quality (e.g., within-habitat variability of resources) can lead to spatially varied demographic rates and aggregation of individuals within suitable patches, thereby increasing differences in the performance of individuals, and ultimately local and regional population persistence (Dhondt et al., 1992; Rodenhouse et al., 1997). It has also been hypothesised that any increase in landscape heterogeneity within a fixed space would lead to a reduction in the average amount of effective area available for individual species, and thus reduce population sizes and increase the likelihood of stochastic extinctions (McKinney, 1997; Kadmon \& Allouche, 2007). Therefore, understanding how spatial variation in habitat quality within patches affects the stability and performance of a population is of particular importance for conserving vulnerable species and maintaining biodiversity in fragmented landscapes.

One important property of landscape heterogeneity relevant to ecological dynamics is the autocorrelation pattern at a variety of spatial scales (Dutilleul \& Legendre, 1993; Harrison \& Bruna, 1999). Species generally exhibit nonstationary patterns in their behaviours, which are explicitly shaped, for the most part, by the spatial pattern of landscape heterogeneity (Boyce, 2006; Guisan et al., 2006). The effect of landscape heterogeneity therefore depends not only on the scale of spatial autocorrelation structure, but also on species habitat requirements and movement capacity (Lovett et al., 2005; Jacobson \& Peres-Neto, 2010). If the scale of spatial autocorrelation is lower, the likelihood of an individual encountering a different environment will increase as it moves away from its present location (Schooley \& Branch, 2007). If the movement range is large, the population becomes well-mixed and hence spatially unstructured (Brachet et al., 1999; Bowler \& Benton, 2005). However, we often have no a priori knowledge about the spatial scale(s) at which animals respond most strongly to spatial heterogeneity of the landscape. Drawing ecological inference about habitat use from observations at one scale may result in misidentification of important ecological covariates and underlying mechanisms relevant to the species of interest (Wiens, 1989; Bradshaw \& Spies, 1992; Boyce et al., 2003; Reding et al., 2013). Coupling ecological processes with landscape heterogeneity across scales is therefore essential to avoid the mismatch between the scale of observations and the 
scale of significant effects of landscape heterogeneity (Wheatley \& Johnson, 2009; de Knegt et al., 2010).

Landscape heterogeneity can be classified as "structural heterogeneity" or "functional heterogeneity", depending on whether or not the measurement is explicitly related to requirements of a particular species or species group (Kolasa \& Rollo, 1991; Fahrig et al., 2011). The former is the complexity or variability of a landscape property identified solely by its physical attributes and without reference to any ecological effect (e.g., land cover type), while the latter is based on the variability of the property that affects ecological processes relevant to the species/community of interest. Ecologically, the spatial pattern of structural heterogeneity is not necessarily equivalent to the pattern of functional heterogeneity and vice versa, because different landscape elements may function similarly for a particular species/group (Lima \& Zollner, 1996; Schick et al., 2008; Fahrig et al., 2011). Therefore, it is essential to know which form of landscape heterogeneity actually affects ecological processes of species of interest and at which scale(s) these effects occur. Such information may greatly improve our knowledge of the spatial nature of species-environment relationships, which is of particular importance for species conservation and management in human-modified landscapes, but few studies have explored this. As landscape fragmentation and modification are likely to increase in the future, we need to understand the relationship of spatial heterogeneity to ecosystem function so as to predict the consequences of alterations in landscape spatial properties.

Moreover, species differ in their biological traits and tolerance to environmental conditions, thereby exhibiting differential responses to local habitat conditions (Jonsen \& Fahrig, 1997; Pigliucci, 2001; Kolasa \& Li, 2003). Given a limited amount of resources available for species in patchy landscapes, we might anticipate that landscape heterogeneity will interact with species' life-history traits in determining species' demographic performance as well as population dynamics (Pulliam \& Danielson, 1991; Mortelliti et al., 2010). Under such circumstances, the question is: how important are the roles of habitat fragmentation, landscape heterogeneity, and their interactions with species' attributes in population persistence? This may require a complex system-style approach where we seek generalizations, emergent properties, and convenient simplifications that enable us to disentangle the consequences of several spatially and temporally varying factors. Further, individual-level variation may also provide clues on the mechanisms underlying population dynamics (Jager, 2001). However, conventional analytical models (i.e., equation-based approaches) cannot take into account discrete individuals and multiple concurrent interactions, which create local population non-uniformity that can affect population dynamics and ecosystem function (Parrott \& Kok, 2000; Getz \& Saltz, 2008). The key 
to understanding the role of landscape heterogeneity in population dynamics is therefore to relate an individual's behaviour and demographic processes to population phenomena in a spatially explicit modelling framework (Wiens et al., 1993b; Wiegand et al., 1999; Wu \& Hobbs, 2002).

\subsection{Research objectives and approaches}

The main objective of this study was to analyse the impacts of landscape heterogeneity on population dynamics and persistence in patchy environments, and to provide general insight into the relationships between population performances and landscape structural and functional properties (e.g., spatial organisation and quality). In doing so, I aimed to assess from a conceptual perspective, how landscape heterogeneity and its interactions with fragmentation affect mobile species with different life-history traits. In addition, I sought to explicitly evaluate the relative roles of different types of landscape heterogeneity on animal space use, based on a large omnivore case study. To accomplish these, I undertook both theoretical simulation modelling and empirical investigations. The realm of landscape heterogeneity is too vast to be included in a single study. In my research, I used the spatial variation in local habitat quality to represent landscape heterogeneity, and my primary focus was on mobile species (e.g., terrestrial animals).

In a series of theoretical studies (Chapter $2-4$ ), I used a spatially explicit, agent-based modelling approach to investigate the population responses of hypothetical mobile species to landscape scenarios of varying heterogeneity and/or fragmentation patterns. Agent-based models (ABMs), also called individual-based models (IBMs), are powerful computational simulation tools and have been increasingly used in a broad range of ecological research related to animal movement and foraging behaviour, population dynamics, and species relationships (see DeAngelis \& Mooij, 2005; Tang \& Bennett, 2010). Specifically, ABMs simulate populations or systems of populations as being composed of discrete agents that represent individual organisms or groups of similar individual organisms, with sets of traits that vary among the agents (Grimm et al., 2006b). ABMs allow the explicit inclusion of individual variation in greater detail than do classical differential-equation and difference equation models (Grimm \& Railsback, 2005). This approach is of particular assistance in landscape-level investigations wherein the challenges of experimental manipulation, control, and replication largely preclude the use of real landscapes.

In an empirical study (Chapter 5), a wavelet-based regression approach was used to investigate the scale-specific space use patterns of American black bears (Ursus americanus) in response to the structural and functional landscape heterogeneity in the Canadian boreal forest. Wavelet analysis 
(Daubechies, 1992) has been applied in research of scale-dependent organism-environment associations (Murwira \& Skidmore, 2005; Carl \& Kühn, 2008; Pittiglio et al., 2013). The wavelet transform decomposes data into scale-specific coefficients related to variations over a set of scales that enables us to quantify spatial structure as a function of both scale and position (Csillag \& Kabos, 2002; Cazelles et al., 2008). More importantly, the wavelet transform is a linear operator, making it possible to use wavelet coefficients within the framework of multiple regressions without altering the interpretation of the regression parameters, i.e., wavelet-coefficient regression (Keitt \& Urban, 2005). This approach differs from traditional methods in that both dependent and independent variables are wavelettransformed prior to regression analysis. A distinct advantage of the waveletcoefficient regression approach is that it measures how a change in one factor at a given scale influences change in another factor at the same scale, which may provide critical clues for understanding the ecological mechanisms underlying animal space use pattern in response to landscape heterogeneity.

\subsection{Thesis outline}

This thesis includes six chapters. Each chapter, except for the introduction and synthesis, has been prepared as an individual paper. These papers have been published in or submitted to peer-reviewed journals. Their structure and content is largely retained in the thesis. The structure of this thesis is as follows:

Chapter 1 states the research background, describes the study objectives, the method used, and outlines the structure of the thesis.

Chapter 2 examines the relative importance of patch size, patch isolation, and spatiotemporal variation in within-patch habitat quality in determining population dynamics in fragmented landscapes.

Chapter 3 explores how spatial pattern of within-patch habitat quality affect population dynamics and long-term persistence in fragmented landscapes.

Chapter 4 tests the joint effects of the scale of spatial autocorrelation in habitat quality and species' life-history traits on population dynamics in spatially heterogeneous landscapes.

Chapter 5 presents a wavelet-based approach to disentangle the roles of structural and functional landscape spatial heterogeneity in animal space use at multiple spatial scales, using a case study of American black bears (Ursus americanus) in a Canadian boreal forest. 
Finally, in Chapter 6, the main results from the previous chapters are brought together to understand the consequences of landscape heterogeneity for populations in patchy environments. The implication of these results for population ecology theory and their potential to inform conservation biology are also discussed.

The literature used in each chapter is combined and placed after the last chapter. 


\section{Within-patch habitat quality determines the resilience of specialist species in fragmented landscapes}

This chapter is based on: Ye, X., Skidmore, A. and Wang, T. (2013) Withinpatch habitat quality determines the resilience of specialist species in fragmented landscapes. Landscape Ecology, 28:135-147. 


\section{Abstract}

Patch geometry and habitat quality among patches are widely recognized as important factors affecting population dynamics in fragmented landscapes. Little is known, however, about the influence of within-patch habitat quality on population dynamics. In this paper, we investigate the relative importance of patch geometry and within-patch habitat quality in determining population dynamics using a spatially explicit, agent-based model. We simulate two mobile species that differ in their species traits: one resembles a habitat specialist and the other a habitat generalist. Habitat quality varies continuously within habitat patches in space (and time). Spatial variation in within-patch quality, together with patch area, controls population abundance of the habitat specialist. In contrast, the population size of the generalist species depends on patch area and isolation. Temporal variation in withinpatch quality is, however, less influential in driving the population resilience of both species. We conclude that specialist species are more sensitive than generalist species to within-patch variation in habitat quality. The patch area-isolation paradigm, developed in metapopulation theory, should incorporate variation in within-patch habitat quality, particularly for habitat specialists.

Keywords: landscape heterogeneity, fragmentation, habitat quality, species specialisation, agent-based model 


\subsection{Introduction}

Understanding the relative importance of different factors affecting fragmented populations is a central question in ecology and conservation (Stacey \& Taper, 1992; Melbourne \& Hastings, 2008). One popular approach for predicting population viability in fragmented landscapes is the 'patch area-isolation" paradigm derived from metapopulation theory (Hanski \& Gaggiotti, 2004; Prugh et al., 2008). This paradigm assumes the landscape to be a binary system of uniformly 'suitable' habitat and 'inhospitable' matrix, for which the probability of species occurrence in habitat patches varies as a function of patch size and isolation (Hanski, 1998; Heaney, 2000).

Though the patch area-isolation paradigm has been validated for small groups of species (e.g. Hanski et al., 1995; Moilanen et al., 1998), the general ability of patch area and isolation to predict species occupancy in patchy environments remains questionable (Prugh et al., 2008; Schooley \& Branch, 2009). A number of empirical studies have shown that the areaisolation paradigm fails to predict the behaviour of populations in fragmented landscapes (Walker et al., 2003; Franken \& Hik, 2004; Fryxell et al., 2005; Pellet et al., 2007). For example, Pellet et al. (2007) analysed multiyear occupancy data for 10 species and found that patch area was not a strong predictor of extinction risk due to a weak correlation between patch area and population size; nor did connectivity generally improve predictions of colonization. Since habitat patches are often heterogeneous and habitat quality influences population size, probability of extinction, and dispersal success, the area-isolation paradigm should be expanded to allow for temporal and spatial variation in habitat quality to improve our understanding of the ecological mechanisms underlying population dynamics in fragmented landscapes (Fleishman et al., 2002; Schooley \& Branch, 2007).

Habitat heterogeneity affects populations through variation in habitat quality among suitable patches, habitat heterogeneity within suitable patches, and matrix heterogeneity (Lovett et al., 2005). There is increasing evidence that habitat quality can be a major driver of population persistence in patchy landscapes (e.g. Clarke et al., 1997; Sutcliffe et al., 1997; Thomas et al., 2001; Franzén \& Nilsson, 2010). For example, Fleishman et al. (2002) found that the quality of habitat patches affected occupancy and turnover rates; Franken and Hik (2004) found habitat quality played a major role in colonization and extinction of local populations; and Ricketts (2001) found that matrix heterogeneity can affect dispersal success and re-colonization of patches following local extinction. However, these studies focused mainly on inter-patch variation in habitat quality in the framework of metapopulation or source-sink dynamics (Pulliam, 1988; Runge et al., 2006), and thus contain little information about the influence of spatial heterogeneity within habitat 
patches on populations. Wiegand et al. (1999; 2005) incorporated three habitat types (good-quality habitat, poor-quality habitat, and matrix) into simulation models and found that both landscape structure and patch quality were important for species with intermediate dispersal abilities.

From a hierarchical point of view, patch-level population dynamics is determined by the within-patch demographic status of population, which is influenced by variation in habitat quality within patches (Pulliam, 1988). Previous simulation studies using empirically derived parameters also suggest that local habitat quality is crucial for the long-term persistence of populations since it strongly affects the probability of extinction (e.g. Root, 1998). For example, Kindvall (1996) found that the degree of habitat heterogeneity within suitable patches can influence the temporal variability of population size and extinction risk of a bush cricket metapopulation. Habitat modification and degradation is likely to increase in the future. Understanding how population dynamics is impacted by temporally and spatially changing habitat quality within/among patches is therefore increasingly important for the conservation of vulnerable species and for maintaining biodiversity. Predictions about the influence of habitat fragmentation and spatial heterogeneity may be inaccurate if they fail to consider conditions within habitat patches. It is, therefore, necessary to address the role of within-patch variation in habitat quality in fragmentation analysis. However, few studies have dealt with the effect of spatial variation within suitable patches on populations in patchy landscapes.

Spatial heterogeneity, often correlated with biophysical conditions of the environment (e.g. microclimate and terrain variability, food availability), forms a mosaic of sites of differing habitat quality within habitat patches. In heterogeneous environments, the spatial variability of local habitat quality leads to spatially varying demographic rates and aggregation of individuals within suitable patches, increasing local densities and competitive pressure on individual performances, i.e. site-dependent population regulation and interference competition (Dhondt et al., 1992; Rodenhouse et al., 1997).

At the temporal scale, habitats can be shaped by many environmental processes (e.g. land cover change, weather fluctuations, human and natural disturbances), producing temporal variation in habitat quality within and among patches (Turner \& Chapin, 2005). Keymer et al. (2000) suggested that a dynamic landscape that is spatially fragmented at a single time-point remains connected over multiple time-steps (i.e. 'direct percolation' phenomenon), thereby promoting population persistence. Similarly, we may expect that temporal fluctuations in habitat quality will buffer spatial variation in habitat quality within suitable patches, ultimately facilitating population habitation. However, the temporal dynamics of habitat quality has mostly 
been studied in the context of habitat destruction-recovery processes, i.e. patch dynamics (e.g. Keymer et al., 2000; Wilcox et al., 2006; Wimberly, 2006). The effect of spatial and temporal variation in habitat quality within patches has not received such attention despite the fact that several studies have demonstrated that within-patch habitat quality can have significant influence on population abundance and survival (see Kindvall, 1996; Lloyd, 2008).

Species differ in their biological traits and tolerance to environmental conditions, and may exhibit different patterns of response to spatial and/or temporal variability in habitat quality (Pigliucci, 2001; Kolasa \& Li, 2003). It is important, therefore, to understand the influence of differences in species niche, and competitive and dispersal abilities on population resilience in heterogeneous landscapes (Mortelliti et al., 2010). A commonly used approach in dealing with differences in species responses to environmental heterogeneity is to incorporate species specialisation (i.e. the 'specialistgeneralist' concept) in the analysis (e.g. Jonsen \& Fahrig, 1997; Tienderen, 1997; Devictor et al., 2008). A specialist species has specific habitat requirements and narrower environmental tolerances than those of a generalist species (Pigliucci, 2001). Understanding responses of different species to within-patch variability is also important for the application of ecological models in conservation planning. Failure to consider differences in species responses may result in misleading inferences about the mechanisms underpinning population declines in heterogeneous environments, ultimately leading to poor management decisions.

In this paper, we use a spatially explicit, agent-based model to examine the effect of within-patch variation in habitat quality on populations in fragmented landscapes differing in patch area and isolation. The ecological motivation for this analysis is to understand how habitat heterogeneity and its interactions with habitat fragmentation patterns affect populations with different life-history traits. Habitat quality in the model varies continuously within habitat patches in space (and time). Simulated populations are generalized to represent territorial species of varying niche breadth and mobility, i.e. specialist vs. generalist species.

\subsection{Methods}

\subsubsection{Model description}

The model simulates the reproduction, movement, and survival of closed populations on landscapes with different patch geometry characteristics and within-patch variation in habitat quality. Our model allows for comparison of the responses of specialist and generalist species to habitat fragmentation 
and within-patch heterogeneity in habitat quality by incorporating patch area, patch isolation, within-patch variations in habitat quality, and species' movement and mortality rate under the concept of 'landscape population

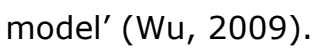

(a)

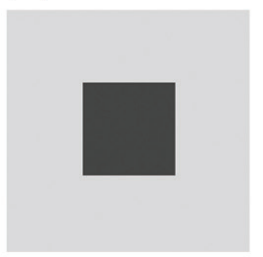

$N_{\text {patch }}=1$ (b)

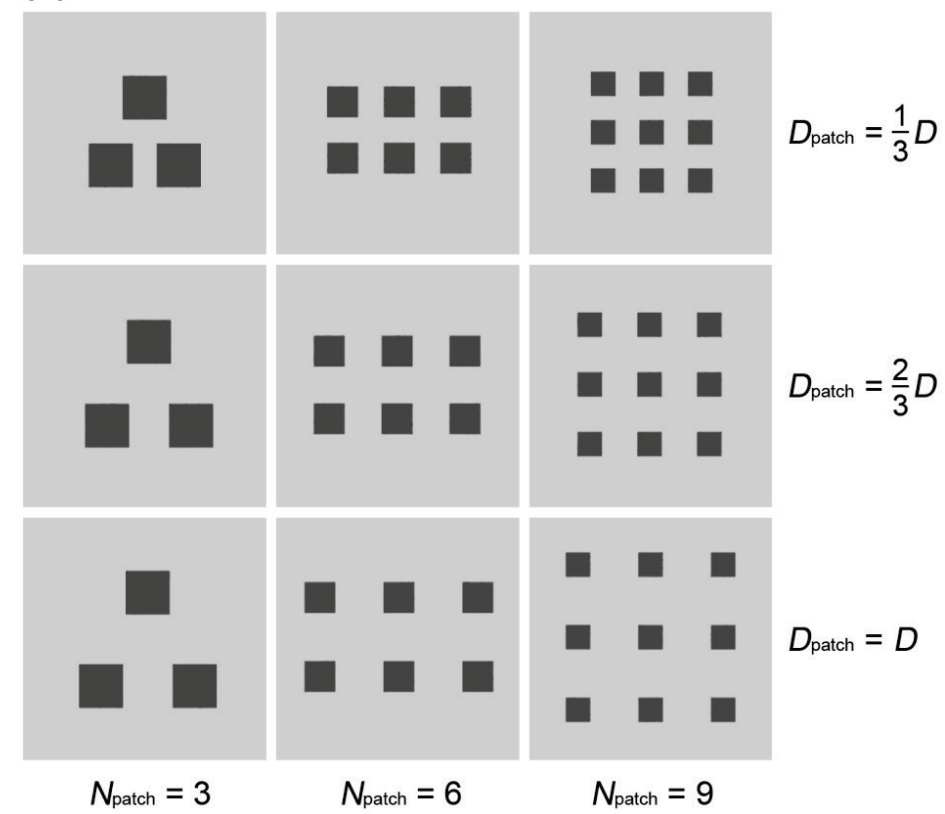

Figure 1 Snapshots of ten simulated landscapes differing in patch geometry. Dark-grey squares represent suitable habitats for species, while light-grey areas represent unsuitable habitats. $N_{\text {patch }}$ is the number of habitat patches, and $D_{\text {patch }}$ is the distance between adjacent patches. $D$ refers to species' maximum movement capacity. The amount of habitat is fixed at $20 \%$ of the landscape area.

The landscape is modelled as a square $100 \times 100$ cells grid consisting of a number of suitable habitat patches within a matrix of unsuitable habitat, with a periodic boundary condition (i.e. the left and right edges and the top and bottom edges of the grid are joined together). Each landscape cell is characterized by its habitat quality $(Q$; range $0-1)$, where habitat quality simply reflects the condition affecting an individual's survival and territoriality. The simulated landscape is, therefore, a continuous representation of habitat quality rather than a binary mosaic of habitat and non-habitat matrix. To work with the patch area-isolation paradigm, a habitat quality of 0.5 is used as the cut-off value for distinguishing suitable cells ('habitat') from unsuitable cells (non-habitat 'matrix'). The amount of habitat is fixed at $20 \%$ of the landscape, in accordance with the theoretical predictions of the threshold for which fragmentation is likely to have a 
significant effect on populations (Fahrig, 1998; Flather \& Bevers, 2002). In order to operate patch area and isolation independently, habitat patches are equally sized and regularly spaced in a landscape (Figure 1). Therefore, changes in the number of patches directly reflect changes in patch area. This design also can diminish unexpected interactions between irregular geometry and species' inter-patch movement (Heinz \& Strand, 2006). In addition, we use the landscape containing one single patch, i.e. with no fragmentation effects, as the baseline case for comparison with fragmented landscapes.
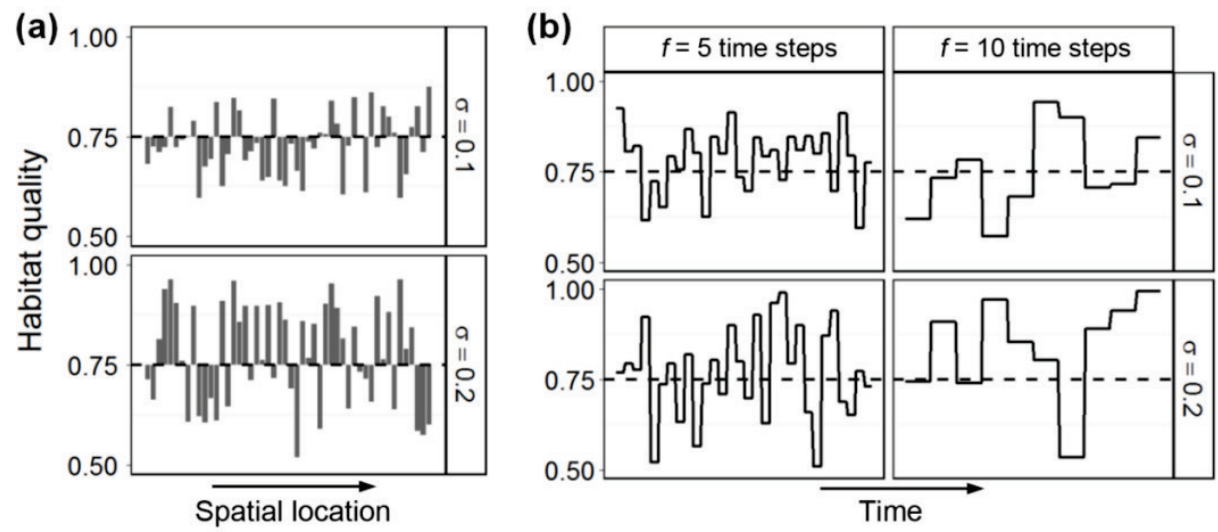

Figure 2 Illustrations of simulated within-patch variations in habitat quality: (a) spatial variation in habitat quality along a straight line across habitat patch and (b) temporal variability in habitat quality of one habitat cell during the simulation period. The symbol $\sigma$ in (a) is the level of spatial variation in habitat quality, and the symbol $f$ in (b) is the temporal frequency of quality updating. Mean habitat quality of habitat cells is maintained at 0.75 during the simulation (depicted by dashed lines).

The central aim of our model is to evaluate how within-patch variations in habitat quality affect population dynamics, where mean habitat quality is constant in space and/or time. We implement within-patch variations in habitat quality by assigning each habitat cell a random value drawn from a normal distribution with mean 0.75 and standard deviation $\sigma$, where $\sigma$ defines the level of within-patch variation in habitat quality. For example, habitat patches are internally homogeneous when $\sigma=0$, but become highly heterogeneous when $\sigma=0.2$ (Figure 2). Because our primary interest is the effect of within-patch variation in habitat quality, we check for the effect of matrix heterogeneity by assigning each matrix cell a random value drawn from a normal distribution with mean 0.25 and standard deviation 0.10 in all simulations. The quality of all habitat cells is restricted to the range $0.5-1$, while the quality of all matrix cells is restricted in the range $0-0.5$.

The population model is a stochastic, single-species model based on the spatio-temporal framework of IBMs proposed by Berec (2002). Only females 
are used in the model, a strategy common to many population models (Pulliam et al., 1992; Wiegand et al., 1999). No age structure is included in the model. Population parameters are adjusted arbitrarily to yield an overall population growth rate $1.05>\lambda>1$ on baseline landscapes with no fragmentation effects or within-patch variation in habitat quality (i.e. $N_{\text {patch }}=$ 1 and $\sigma=0$ ).

The processes of movement (including territoriality), reproduction, and mortality drive population dynamics in the landscape. At every time-step, individuals may reproduce, move, or die. The order of these events is randomized for each individual at each time-step. All demographic processes were implemented based on probabilities. For example, if the probability of mortality of an individual is 0.3 , then it dies only if the generated random number (belonging to a uniform probability density in the range $0-1$ ) is less than 0.3 .

Both movement and reproduction are density-independent. Per time-step, dispersing individuals are only allowed a limited number of spatial steps (i.e. move to neighbouring cells) in search of an acceptable home range. However, they cannot enter cells where habitat quality is lower than a threshold $Q^{\prime}$ (i.e. habitat-based random walk). The assumption of random walk seems to be unrealistic for species that may actively search the landscape for home range, but is conservative in the sense that effects of fragmentation are easy to detect (Fahrig, 1998). Only residents (i.e. individuals with home range) can reproduce, with a fixed probability.

Habitat quality and local density affect population dynamics by modifying individuals' mortality rates: $M=M_{0} \times\left(1+\exp \left(-C \times(Q / J)^{2}\right)\right)$, where $M_{0}$ is the basic mortality rate, $C$ is the niche breadth coefficient, $Q$ is the habitat quality of the occupied cell, and $J$ is the number of individuals within an area of $5 \times$ 5 cells surrounding the occupied cell. The niche breadth coefficient defines the sensitivity of the mortality rate to habitat quality (see Appendix 1). We assumed that only the mortality rate is associated with local habitat quality and population density. More complex relations, e.g., making both mortality and reproduction functions of habitat quality, would by virtue of Jensen's inequality prohibit comparison between landscapes (Ruel \& Ayres, 1999; Stoddard, 2010). 


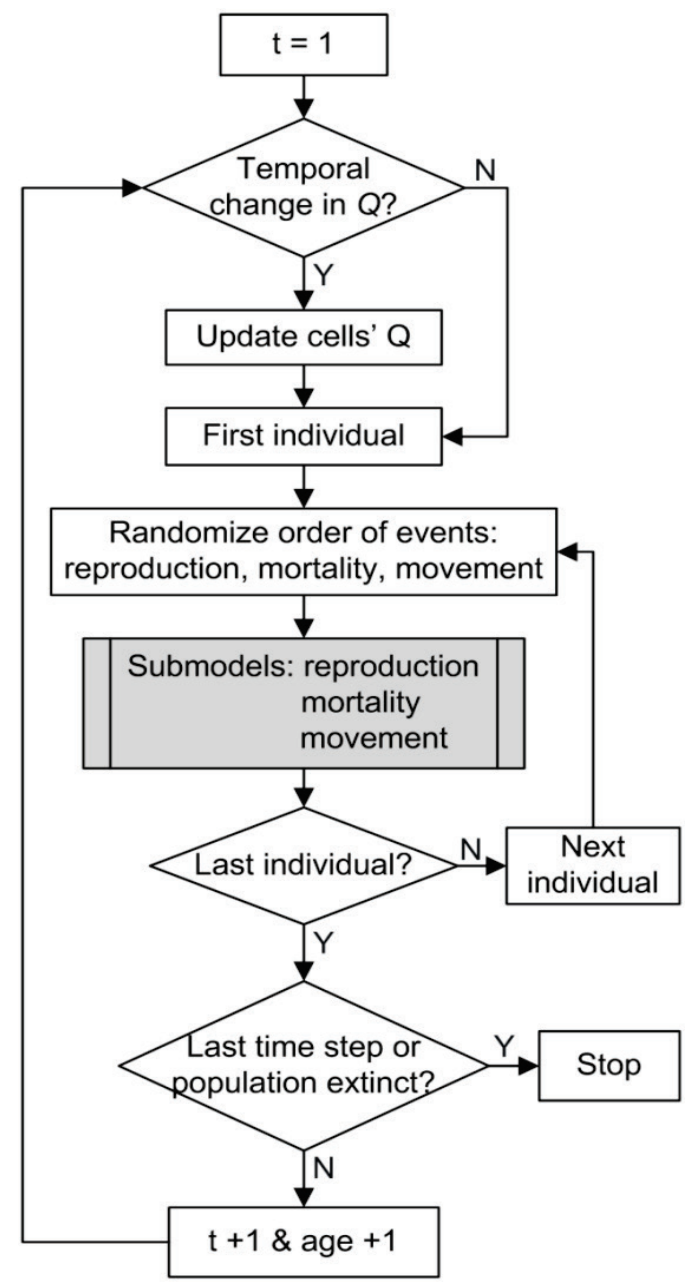

Figure 3 Flow diagram of the main routine that determines the dynamics of a population in the model. Submodels for reproduction, mortality, and movement within a time step refer to Appendix 1.

In our model, we simulated two types of species that differ in niche breadth and movement capacity: one representing specialist species and one generalist species. The generalist species has a broader niche breadth and greater movement capacity than the specialist species (Tienderen, 1997; Pigliucci, 2001). Overviews of the model structure and its parameters are given in Figure 3 and Table 1, respectively. A detailed description of the model following the ODD protocol is given in Appendix 1. The ODD (Overview, Design concepts, and Details) protocol is designed to facilitate the readability and completeness of the description of individual- and agentbased models (Grimm et al., 2006a). 
Table 1 Variables, parameters, and constants used in the model.

\begin{tabular}{|c|c|}
\hline Parameter (notation) & Values \\
\hline \multicolumn{2}{|l|}{ Landscape } \\
\hline Landscape size & $100 \times 100$ cells \\
\hline Amount of suitable habitat in the landscape & $20 \%$ \\
\hline Number of suitable habitat patches $\left(N_{\text {patch }}\right)$ & $1,3,6,9$ \\
\hline Distance between nearest habitat patches $\left(D_{\text {patch }}\right)$ & $\frac{1}{3} \cdot D, \frac{2}{3} \cdot D, D$ \\
\hline Range of habitat quality $(Q)$ & $0-1$ \\
\hline Cut-off quality value for habitat and matrix & 0.5 \\
\hline Qualities of habitat cells & $\begin{array}{l}\text { Normal distribution } N \\
(0.75, \sigma)\end{array}$ \\
\hline Within-patch variation in habitat quality $(\sigma)$ & $0,0.1,0.2$ \\
\hline Qualities of matrix cells & $\begin{array}{l}\text { Normal distribution } N \\
(0.25,0.10)\end{array}$ \\
\hline \multicolumn{2}{|l|}{ Population model } \\
\hline Maximum life span & 20 time steps \\
\hline Reproduction probability & 0.8 \\
\hline Probability of a litter of $i$ cubs $\left(L_{i}\right)$ & $L_{1}=0.8, L_{2}=0.2$ \\
\hline Basic mortality $\left(M_{0}\right)$ & 0.25 \\
\hline Niche breadth coefficient $(C)^{*}$ & $C_{S}=3, C_{G}=5$ \\
\hline Maximum movement capacity $(D)^{*}$ & $\begin{array}{l}D_{\mathrm{S}}=20 \text { cells, } D_{\mathrm{G}}=30 \\
\text { cells }\end{array}$ \\
\hline $\begin{array}{l}\text { Minimum habitat quality that can be tolerated } \\
\left(Q^{\prime}\right)^{*}\end{array}$ & $Q^{\prime}{ }_{S}=0.25, Q_{G}^{\prime}=0.20$ \\
\hline Home range size & $3 \times 3$ cells \\
\hline $\begin{array}{l}\text { Minimum habitat quality of acceptable home } \\
\text { range }\left(Q_{\text {range }}\right)\end{array}$ & $\begin{array}{l}6.5 \text { (sum of } 9 \text { cells' } \\
\text { quality) }\end{array}$ \\
\hline Probability of abandonment of home range & 0.1 \\
\hline \multicolumn{2}{|l|}{ Model simulation } \\
\hline Starting number of individuals & 200 \\
\hline Maximum time-steps in simulation & 300 \\
\hline Time intervals of habitat quality updating $(f)^{* *}$ & 5,10 time-steps \\
\hline
\end{tabular}

\subsubsection{Simulation experiments}

We designed two different simulation experiments to investigate how the population responds to variation in within-patch quality under various scenarios of fragmentation. In the first experiment, we examined the effect of spatial variation in within-patch quality on population dynamics. Simulated spatial variation in within-patch quality is constant during population simulations (this is termed the 'static' landscape). In addition to baseline 
scenarios $\left(N_{\text {patch }}=1\right)$, the combination of patch area, isolation ( 9 landscapes), and within-patch variation in quality $(\sigma=0,0.1,0.2)$ produced 27 scenarios $(3 \times 3 \times 3=27)$ in this experiment.

In the second experiment, the 'static' landscape becomes temporally dynamic (termed as 'dynamic' landscape) during population simulations. For all landscapes with within-patch variation in habitat quality (i.e. $\sigma \neq 0$ ), the quality of each cell is reassigned at regular intervals using the same method as for generating variations in habitat quality (see section 'Model description' for more details). We simulate two different time intervals ( $f=5,10$ timesteps; see Table 1) for updating quality under the consideration of species' maximum life span (20 time-steps). When $f=5$ time-steps, species experiences rapid changes in habitat quality (e.g. frequently disturbed habitat), while in the case of $f=10$ time-steps, species live in relatively 'stable' habitats (Figure 2). The combination of patch area, isolation (9 landscapes), spatial ( $\sigma=0.1,0.2)$ and temporal ( $f=5,10$ time-steps) within-patch variations in habitat quality created 36 'dynamic' landscape scenarios for the second experiment $(3 \times 3 \times 2 \times 2=36)$.

\subsubsection{Model operation}

The simulation model was implemented in the NetLogo 4.1.2 environment (Wilensky, 1999). For each species, we performed 30 replicate runs for each landscape scenario. At the beginning of each run of the model, the landscape is initialized based on parameters entered. Thereafter, 200 individuals are distributed randomly among habitat patches in the landscape. All simulations last for 300 time-steps or until the population become extinct. This is based on the preliminary runs using 500 time-steps, in which we found that any population either reached a state of dynamic equilibrium or became extinct within the first 300 time-steps. For each run in which the population persisted, population size (primary response variable) was recorded as the mean of the last 50 time steps. We also monitored proportion of residents, mortality rate of residents, and mortality rate of dispersers for both species for better understanding of underlying mechanisms involved in the regulation of population dynamics.

\subsubsection{Statistical analysis}

We used Wilcoxon Mann-Whitney test to determine if there were significant differences in responses between two hypothetical species subjected to landscape fragmentation and within-patch variation in habitat quality. For each species, we used factorial analysis of variance (ANOVA) to examine the effects of number of patches, distance between patches, and within-patch variation in habitat quality on population size. Factorial ANOVA is a flexible 
analytic technique when there are two or more independent variables in the design (Box et al., 2005), and allows us to analyse the main effect of each factor and the effect of each combination of the levels of factors on the response variable. Percentage of variance explained and generalized etasquared (Olejnik \& Algina, 2003; Bakeman, 2005) were used for measuring the importance of the effects of the factors and their interactions (see Appendix 2 for the calculation of generalized eta-squared). Prior to analysis, population size was log-transformed to improve data normality. For the analysis of 'dynamic' landscapes, we used a combined factor (" $\left.\sigma-f^{\prime \prime}\right)$ to represent the joint influence of spatial variation in habitat quality and temporal frequency of changes in habitat quality, i.e. spatio-temporal variation in habitat quality. All data analyses were performed using the statistical software R, version 2.14.1 (R Development Core Team, 2011).

\subsection{Results}

For both the 'static' and 'dynamic' simulation experiments, no extinction events were observed. We therefore focused our analysis on the effects of patch area, isolation, and within-patch variations in habitat quality on population sizes of hypothetical specialist and generalist species.

\subsubsection{Experiment 1: species in 'static' landscapes}

As we expected, there were significant differences in population size between the hypothetical specialist species and generalist species in all landscape scenarios except scenarios with $N_{\text {patch }}=3$ and $\sigma=0$ and scenarios with $N_{\text {patch }}$ $=6$ and $\sigma=0.2$ (Mann-Whitney test, $P<0.05$ ). The generalist species had greater decline in population size than the specialist species in response to decreased patch area, while the specialist species decreased more rapidly in population size as spatial variation in habitat quality increased (Figure 4). Interestingly, the proportions of residents for the specialist species were lower than the generalist species in all landscape scenarios (Mann-Whitney test, $P<0.01$ ). In addition, the specialist species experienced a higher resident mortality rate, while the generalist species is subjected to a higher disperser mortality rate (Mann-Whitney test, $P<0.05$ for both). 

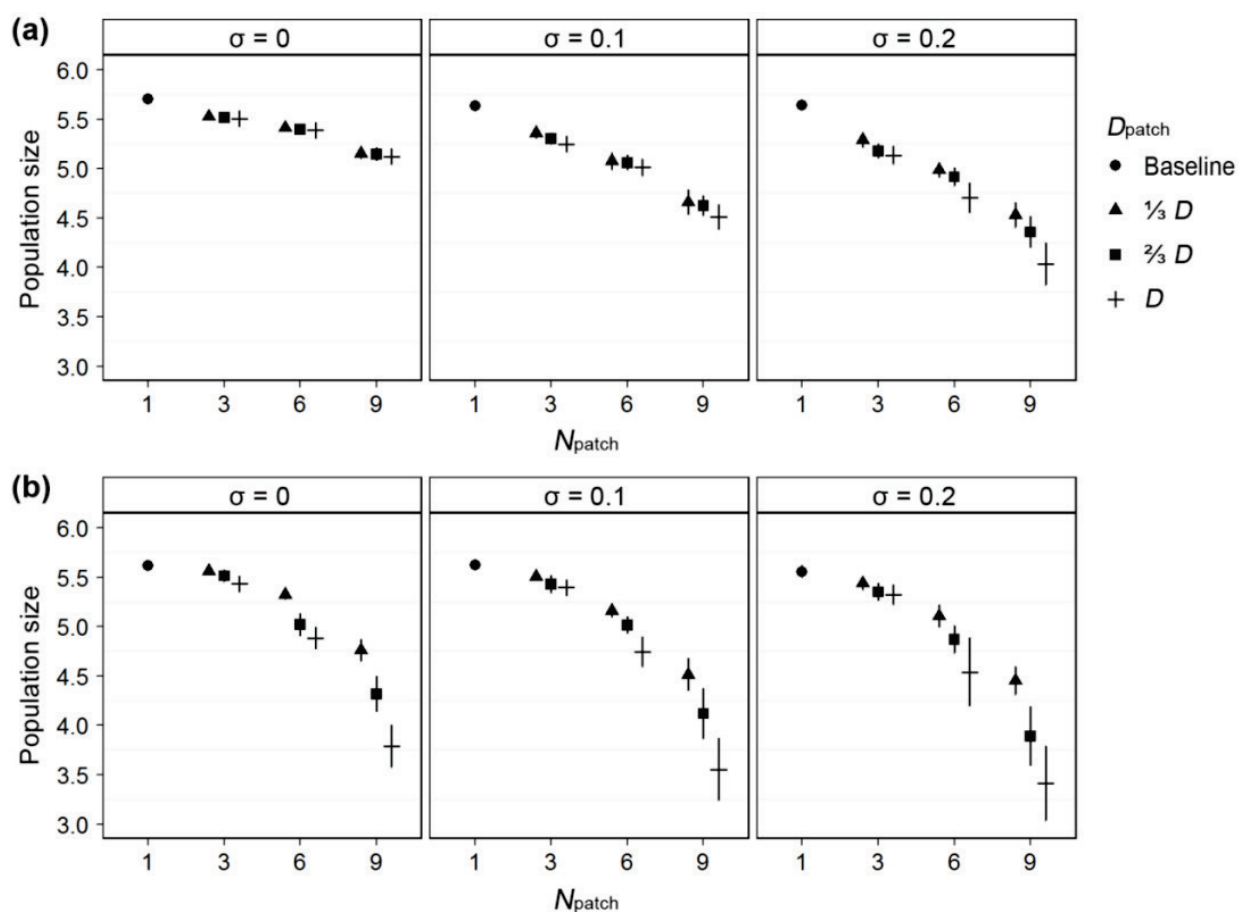

Figure 4 Mean population size of the (a) hypothetical specialist and (b) hypothetical generalist species on 'static' landscapes. $\boldsymbol{N}_{\text {patch }}$ is the number of patches, $D_{\text {patch }}$ is the distance between patches, and $\sigma$ is the spatial variation in habitat quality. $D$ refers to species' maximum movement capacity. Population sizes were log-transformed and plotted with $95 \%$ confidence intervals (missing error bars indicate a confidence interval too small to display).

The effects of patch area, isolation, and within-patch variation in habitat quality varied distinctly between the two hypothetical species (Table 2). For the specialist species, its population size declined significantly with decrease in patch area and increase in within-patch variation in habitat quality (Figure 4a). Patch area and within-patch variation in habitat quality both had strong impacts, accounting for $\sim 36 \%$ and $\sim 26 \%$, respectively, of the variation in population size (Table $2 a$ ). In contrast, patch isolation explained only $\sim 2 \%$ of the variation in population size, negatively affecting the population size only in combination with within-patch variation in habitat quality $\left(F_{2,807}=7.41, P\right.$ $<0.001)$. Increases in within-patch variation in habitat quality also aggravated the impact of patch area on the specialist species $\left(N_{\text {patch }} \times \sigma ; F_{8}\right.$, $801=195.75, P<0.001)$, showing greater declines in population size as patch area decreased (Figure $4 a$ ). Both the proportion of residents and its mortality rate increased as within-patch variation in habitat quality increased (data not shown). 
For the generalist species, patch area became the dominant factor in driving its population dynamics, accounting for $\sim 52 \%$ of the variation in population size (Table 2b). Patch isolation also had an appreciable impact on the species $\left(F_{2,807}=33.96, P<0.001\right)$, explaining $\sim 8 \%$ of the variation in population size. Patch isolation further exacerbated the impact of patch area on the generalist species $\left(N_{\text {patch }} \times N_{\text {patch }} ; F_{8,801}=174.65, P<0.001\right)$, resulting in greater decreases in population size as patch area decreased (Figure $4 \mathrm{~b}$ ). The main effect of within-patch variation in habitat quality was also significant $\left(F_{2}\right.$, $807=6.95, P<0.01)$, but accounted only for $\sim 2 \%$ of variation in population size. Similarly, both proportion of residents and mortality rate of residents increased with increasing in within-patch variation in habitat quality (data not shown).

Table 2 Factorial ANOVAs on population size of the (a) hypothetical specialist and (b) hypothetical generalist species in landscapes differing in number of patches, distance between patches, and spatial variation in habitat quality within patches.

\begin{tabular}{|c|c|c|c|c|c|}
\hline Source of variation & $d f$ & Percentage of $S S_{T}$ & $F$ & $P$ & $\eta_{\mathrm{G}}^{2}$ \\
\hline \multicolumn{6}{|l|}{ (a) hypothetical specialist } \\
\hline$N_{\text {patch }}$ & 2 & 36.14 & 329.60 & $<0.001$ & 0.55 \\
\hline$D_{\text {patch }}$ & 2 & 1.80 & 33.18 & $<0.001$ & 0.06 \\
\hline$\sigma$ & 2 & 26.27 & 419.71 & $<0.001$ & 0.47 \\
\hline$N_{\text {patch }} \times D_{\text {patch }}$ & 4 & 0.31 & 2.02 & 0.096 & 0.01 \\
\hline$N_{\text {patch }} \times \sigma$ & 4 & 3.75 & 24.42 & $<0.001$ & 0.11 \\
\hline$D_{\text {patch }} \times \sigma$ & 4 & 1.19 & 8.48 & $<0.001$ & 0.04 \\
\hline $\begin{array}{l}N_{\text {patch }} \times D_{\text {patch }} \times \sigma \\
\text { (b) hypothetical generalist }\end{array}$ & 8 & 0.38 & 1.23 & 0.284 & 0.01 \\
\hline$N_{\text {patch }}$ & 2 & 52.20 & 806.66 & $<0.001$ & 0.60 \\
\hline$D_{\text {patch }}$ & 2 & 7.76 & 89.04 & $<0.001$ & 0.18 \\
\hline$\sigma$ & 2 & 1.69 & 30.43 & $<0.001$ & 0.05 \\
\hline$N_{\text {patch }} \times D_{\text {patch }}$ & 4 & 3.60 & 21.34 & $<0.001$ & 0.09 \\
\hline$N_{\text {patch }} \times \sigma$ & 4 & 0.29 & 1.86 & 0.122 & 0.01 \\
\hline$D_{\text {patch }} \times \sigma$ & 4 & 0.05 & 0.28 & 0.893 & $<0.01$ \\
\hline$N_{\text {patch }} \times D_{\text {patch }} \times \sigma$ & 8 & 0.09 & 0.22 & 0.988 & $<0.01$ \\
\hline
\end{tabular}

Note: $N_{\text {patch }}$ is the number of patches; $D_{\text {patch }}$ is distance between nearest patches; $\sigma$ is spatial variation in habitat quality. $S S_{T}$ is total sum of squares; $\eta_{G}^{2}$ is generalized eta-squared. 


\subsubsection{Experiment 2: species in 'dynamic' landscapes}

When simulating in 'dynamic' landscapes, no significant differences in population size between two species were observed in 17 out of 36 landscape scenarios, for instance, in scenarios with $N_{\text {patch }}=6$ and $D_{\text {patch }}=1 / 3 \cdot D$ and scenarios with $N_{\text {patch }}=9$ and $D_{\text {patch }}=1 / 3 \cdot D$ (Mann-Whitney test, $P>0.05$; Figure 5). The specialist species still had a lower proportion of residents than the generalist species in all landscape scenarios, and the generalist species had a higher disperser mortality rate (Mann-Whitney test, $P<0.01$ for both).

In 'dynamic' landscapes, patch area was the most important factor in driving population size, explaining $\sim 52 \%$ of the variation for both hypothetical species (Table 3 ). Patch isolation explained $\sim 5.5 \%$ and $\sim 7.2 \%$ of the variation for the specialist species and generalist species, respectively. Patch isolation also aggravated the negative effect of patch area $\left(N_{\text {patch }} \times N_{\text {patch }} ; F_{8}\right.$, $1611[\mathrm{~S}]=261.14, F_{8,1611[\mathrm{G}]}=326.05$, both $\left.P<0.001\right)$, resulting in increasing losses in population size for both species as patch area decreased (Figure 5).
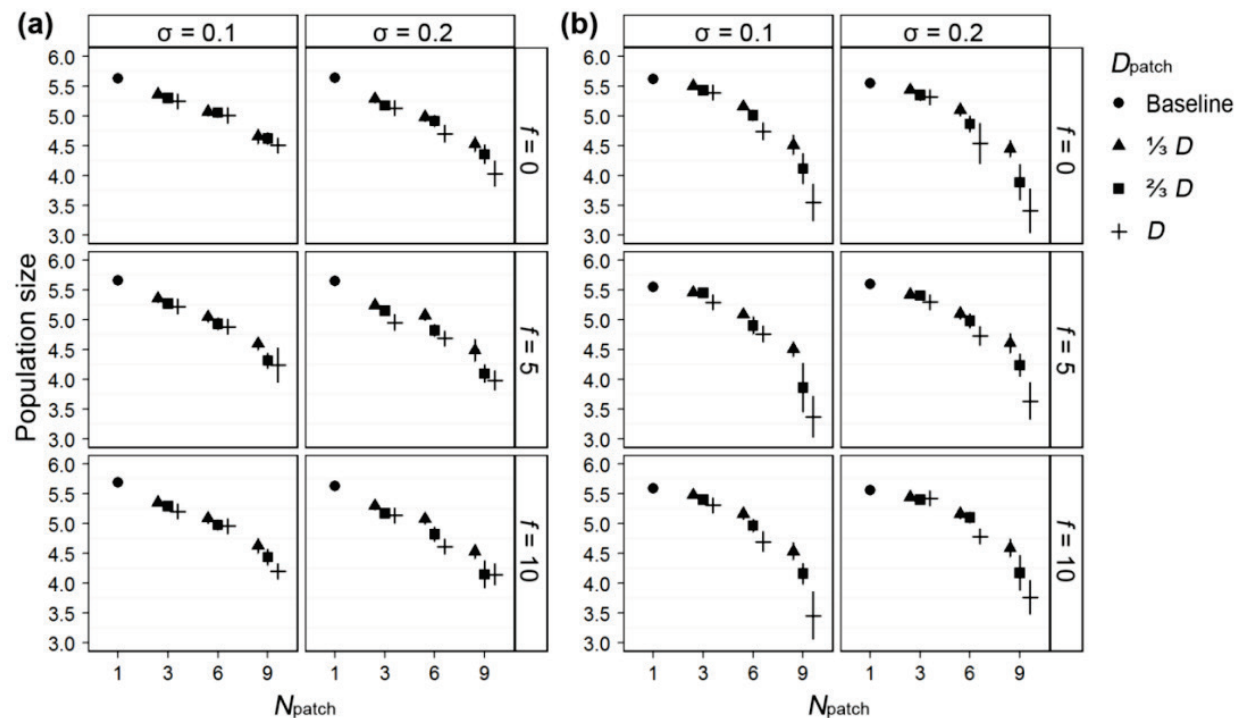

Figure 5 Mean population size of the (a) hypothetical specialist and (b) the hypothetical generalist species on 'dynamic' landscapes. $\boldsymbol{N}_{\text {patch }}$ is the number of patches, $D_{\text {patch }}$ is the distance between patches, $\sigma$ is the spatial variation in habitat quality, and $f$ is the temporal variation in habitat quality. $D$ refers to species' maximum movement capacity. Population sizes were log-transformed and plotted with $\mathbf{9 5 \%}$ confidence intervals (missing error bars indicate a confidence interval too small to display).

The effect sizes of spatio-temporal variation in habitat quality were low for both species (Table 3 ). Increasing spatio-temporal variation in habitat quality 
generally decreased the population size of the specialist species $\left(F_{5,1614}=\right.$ $10.11, P<0.001)$, but had little effect on population size of the generalist species $\left(F_{5,1614}=1.56, P=0.17\right)$. However, high level of spatio-temporal variation in habitat quality $(\sigma=0.2$ and $f=10)$ did mitigate the impact of patch area and isolation on the generalist species (Tukey's HSD test, $P<$ $0.05)$, resulting in appreciable but relatively small promotion in population size on highly fragmented habitats (Figure $5 b$ ).

Table 3 Factorial ANOVAs on population size of the (a) hypothetical specialist and (b) hypothetical generalist species in landscapes differing in the number of patches, distance between patches, and spatio-temporal variation in habitat quality within patches.

\begin{tabular}{lrrrrr}
\hline Source of variation & $d f$ & Percentage of $S S_{\mathrm{T}}$ & $F$ & $P$ & $\eta_{\mathrm{G}}^{2}$ \\
\hline (a) hypothetical specialist & & & & & \\
$N_{\text {patch }}$ & 2 & 51.18 & 1033.11 & $<0.001$ & 0.57 \\
$D_{\text {patch }}$ & 2 & 4.71 & 94.93 & $<0.001$ & 0.11 \\
$\sigma-f$ & 5 & 3.03 & 24.50 & $<0.001$ & 0.07 \\
$N_{\text {patch }} \times D_{\text {patch }}$ & 4 & 0.58 & 5.89 & $<0.001$ & 0.02 \\
$N_{\text {patch }} \times \sigma-f$ & 10 & 0.51 & 2.07 & 0.023 & 0.01 \\
$D_{\text {patch }} \times \sigma-f$ & 10 & 0.72 & 2.91 & 0.001 & 0.01 \\
$N_{\text {patch }} \times D_{\text {patch }} \times \sigma-f$ & 20 & 0.49 & 0.98 & 0.481 & $<0.01$ \\
$(b)$ hypothetical generalist & & & & & \\
$N_{\text {patch }}$ & 2 & 51.05 & 1076.96 & $<0.001$ & 0.58 \\
$D_{\text {patch }}$ & 2 & 7.13 & 150.52 & $<0.001$ & 0.16 \\
$\sigma-f$ & 5 & 0.48 & 4.07 & 0.001 & 0.01 \\
$N_{\text {patch }} \times D_{\text {patch }}$ & 4 & 3.64 & 38.40 & $<0.001$ & 0.09 \\
$N_{\text {patch }} \times \sigma-f$ & 10 & 0.29 & 1.23 & 0.267 & $<0.01$ \\
$D_{\text {patch }} \times \sigma-f$ & 10 & 0.13 & 0.55 & 0.856 & $<0.01$ \\
$N_{\text {patch }} \times D_{\text {patch }} \times \sigma-f$ & 20 & 0.17 & 0.35 & 0.997 & $<0.01$ \\
\hline
\end{tabular}

Note: $N_{\text {patch }}$ is the number of patches; $D_{\text {patch }}$ is distance between nearest patches; $\sigma-f$ is the joint effect of spatial variation in habitat quality and temporal frequency of changes in habitat quality; $S S_{T}$ is total sum of squares; $\eta_{G}^{2}$ is generalized eta-squared.

\subsection{Discussion}

Our simple model of population dynamics in complex landscapes shows explicitly that landscape fragmentation and within-patch variation in habitat quality have important consequences for population dynamics, depending on 
species' life history attributes. In general, patch area and within-patch variation in habitat quality is critical to the populations of specialist species, while generalist species was more sensitive to patch area and isolation, showing great declines as habitat fragmentation increased. The generalist species had a higher probability of occupying territory than the specialist species, but suffered a higher dispersing mortality rate in all landscape scenarios. These species-specific responses to fragmentation and local spatial heterogeneity reveal underlying limitations imposed by their distinct species characteristics. Habitat fragmentation can severely restrict the spread of terrestrial species, especially those specialists with poor dispersing capacity (Wiegand et al., 2005), thus decreasing the probability of finding suitable territory. Fail to finding suitable habitat will decrease individual's fitness (e.g. reproduction success) and increase intraspecies competition. Consequently, dynamics in their populations rely heavily on local habitat quality. In contrast, the generalists can have a higher probability of occupying suitable territory under the same level of fragmentation, decreasing their dependency on local habitat quality. However, if individuals spend more time in unsuitable area (i.e. matrix) due to habitat fragmentation, they will suffer high dispersal mortality, leading to great declines in populations.

Although the model was conservative, due to a number of assumptions, our results are largely consistent with prior studies that report species' traitsdependent susceptibility or resilience to fragmentation (Davies et al., 2000; Henle et al., 2004; Ewers \& Didham, 2006). Empirical studies on the effects of patch area, isolation, and habitat quality have produced contrasting results regarding which factors play a major role in determining population dynamics. Some studies suggest that patch size and connectivity are more important (e.g. Vögeli et al., 2010), whereas others indicate the quality of the habitat patch has a greater effect on populations than patch size and isolation (e.g. Franken \& Hik, 2004; Donner et al., 2010). In view of our results, these conflicting findings actually highlight the need to recognize species specialization as a dimension relevant to testing the effects of habitat fragmentation and spatial variability in habitat quality. By explicitly considering the effect of species traits, it becomes possible to explain many seemingly contradictory results in the fragmentation literature (Ewers \& Didham, 2006).

Spatial variation in habitat quality within patches had a much stronger impact on the specialist species than the generalist species, indicating that the specialists may be susceptible to local variability within habitats. We observed that population size of the specialist species decreased as withinpatch variation increased, which differs from the findings of Kindvall (1996) that the extinction risk of a bush cricket metapopulation was higher on homogeneous habitat patches than heterogeneous patches. However, 
empirical testing of the hypothesis that populations are less variable in size on heterogeneous than homogeneous patches is still lacking. It is hard to judge the general applicability of these finding. Nevertheless, several studies (Kindvall, 1996; Lloyd, 2008) have already shown that within-patch habitat quality had significant influence on population abundance and survival. Habitat quality may be more relevant than patch connectivity in the population dynamics of habitat specialists, even for those with relatively high dispersal abilities, but the degree of association for each factor may vary under different scenarios.

Similarly, there is little research examining the effect of spatio-temporal variation in habitat quality on population dynamics. For this reason, we cautiously compared our results with other studies addressing habitat creation-destruction dynamics, because these kinds of dynamics can be considered as an extreme case of variation in habitat quality. Keymer et al. (2000) simulated habitat dynamics as a Markov process with constant probabilities of habitat creation-destruction and found that increased habitat dynamics always resulted in decreased habitat occupancy of the dispersallimited species. Matlack and Monde (2004) used a similar Markov model but found that habitat occupancy of species with rapid dispersal rates peaked at intermediate rates of habitat dynamics. In our simulations, increasing spatiotemporal variation in habitat quality had negative impact on population size of the specialist species, but had little effect on population size of the generalist species. It is likely that this is related to differences in their movement ability and habitat tolerance. For instance, the generalist species had a higher dispersal capacity than the specialist species, which allows the generalist species to find suitable territory when facing spatially heterogeneous and temporally dynamic environments. It is also possible that the frequency of temporal changes in habitat quality is too low (compare to species' life span) in the sense that effects of spatio-temporal variation are hard to detect. Furthermore, our approach simulates temporal variation in habitat quality as discrete, ephemeral disturbances, whereas temporal dynamics in real landscapes is a continuous, scale-dependent process. Our study should be viewed, therefore, as a basis for future studies that consider simultaneously the effects of spatial and temporal stochasticity on populations in heterogeneous environments.

In our model, the generalist species was more vulnerable to patch isolation than the specialist species, showing greater declines in population size when subjected to same level of patch area and within-patch condition, as shown in Figure 4. This result seems to be counter-intuitive, as species with better mobility are more likely to survive in fragmented landscapes than species with low mobility (Nieminen, 1996; Brachet et al., 1999). However, the probability of successful inter-patch movement does not depend only on the 
species' movement ability but also on inter-patch distance, while the survival rate of dispersers depend on matrix quality (Fahrig, 2001). If movement is not directed, individuals are likely to spend more time in uninhabitable area as habitat fragmentation increase, resulting in high dispersal mortality. It is suggested that species with 'intermediate' dispersal abilities are most sensitive to patch isolation, largely because intermediate mobility resulted in individuals leaving one fragment but failing to reach the next, in turn leading to an overall increase in mortality (Fahrig, 1998; Wiegand et al., 1999; Thomas, 2000; Fraterrigo et al., 2009). Our results are comparable to these findings, despite differing modelling approaches. For instance, the generalist species in our model experienced higher dispersing mortality rate than the specialist species in all simulated landscapes. Such differences imply that one should carefully consider the influence of species traits when interpreting the effect of patch isolation. For example, depending on species' dispersal capability and perceptions of the matrix quality, landscapes that are fragmented for one species may be continuous for others (Diffendorfer et al., 1995; Stouffer \& Bierregaard, 1995).

Differential responses of species to habitat quality and spatial configuration may impose different selective pressures and require different strategies for survival and dispersal (Pandit et al., 2009). When a landscape alters abruptly, species with narrow ecological niches tend to be at higher risk of extinction (Sodhi et al., 2009; Clavel et al., 2010), especially for large-bodied specialists that require larger home ranges and more resources than small ones (Cardillo et al., 2005). Habitat restoration and rejuvenation (Fischer \& Lindenmayer, 2007), together with connectivity where necessary, may be an effective strategy for the long-term conservation of biodiversity.

\section{Acknowledgements}

We are grateful for the insightful comments of Dr. T. Wiegand and two anonymous reviewers. This work was supported by the Erasmus Mundus External Co-operation Window (EMECW) Programme of the European Union and co-funded by the ITC Research Fund. 


\section{Appendix 1}

\section{Description of the agent-based model}

The model description follows the ODD protocol (overview, design concepts, and details) for describing individual- and agent-based models proposed by Grimm et al. (2010; 2006).

\section{Purpose}

The model was created to understand how within-patch variations in habitat quality interact with habitat fragmentation (characterized by patch area and isolation) in determining the population dynamics of specialist and generalist species. The model was designed to compare responses of different species to the effects of habitat fragmentation and within-patch heterogeneity rather than to precisely simulate the population dynamics of specific species.

\section{Entities, state variables, and scales}

We built a spatially explicit, agent-based system that a single-species population lives in a landscape containing a number of habitat patches embedded in an inhospitable matrix. The landscape is modelled as a $100 \times$ 100 square cells grid, with a periodic boundary condition, and its spatial patterns are controlled by the following variables: the amount of habitat, number of habitat patches, distance between adjacent habitat patches, and within-patch variation in habitat quality. Cell size was not specified. Instead, we defined an acceptable home range for an individual as being a $3 \times 3$ cell block. Individuals of populations are characterized by the following state variables: identity number, spatial location ( $x-y$ coordinates), demographic parameters (e.g. reproductive rate, mortality rate, no. of offspring), status (resident or disperser), niche breadth, and movement capacity. Time runs in discrete steps and the simulation lasts 300 time-steps or until the population becomes extinct. A complete set of variables relevant to the model are listed in Table 1.

\section{Process overview and scheduling}

For each time-step, individuals may die, reproduce, or move (Figure 3 ). The order of the three events is randomized for each individual at each time step. The mortality rate is individual-specific, depending on local habitat quality and population density. Only resident individuals are capable of reproducing with a constant probability. New-born individuals are not subject to the mortality rate of the actual time-step at birth; they become adults upon commencement of the following time-step. 


\section{Design concepts}

Emergence - Population dynamics emerge from individuals' behaviours (reproduction, dispersal and mortality) and interactions among individuals.

Sensing - Individuals are assumed to be capable of distinguishing spatial cells that can be accessed (i.e. ability to detect barriers to movement).

Interaction - Individuals compete for space to establish their territory. An individual cannot establish a home range on spatial cells that already constitute the home range of other individuals.

Stochasticity - All modelled processes are implemented stochastically: random order of individuals in the main routine; random order of reproduction, mortality, and movement events; and randomly chosen neighbouring cell for individual to move in. Reproduction and mortality events are determined using probabilities. For example, if an individual's probability of mortality is 0.3 , then it dies only if the generated random number, belonging to a uniform probability density in the range $0-1$, is less than 0.3 . Furthermore, the habitat qualities of cells are drawn randomly from normal distributions. The initial spatial position of individuals in habitat patches is also randomly chosen.

Observation - The following parameters are observed for both species: population size, proportion of residents, mortality rate of residents, and mortality rate of dispersers.

\section{Initialization}

Square habitat patches are equally sized and evenly spaced in the landscape in order to operate patch area and isolation independently. Habitat quality of each habitat cell is drawn from the normal distribution $N(0.75, \sigma)$, while the habitat quality of each matrix cell is drawn from the normal distribution $N(0.25,0.10)$. After creating a landscape, two hundred individuals will distribute randomly within habitat patches.

\section{Input}

For cases that $\sigma \neq 0$, each cell's habitat quality changes at a given intervals $(f)$. The values assigned to cells are generated randomly from the normal distribution characterized by the value of $\sigma$. 


\section{Submodels}

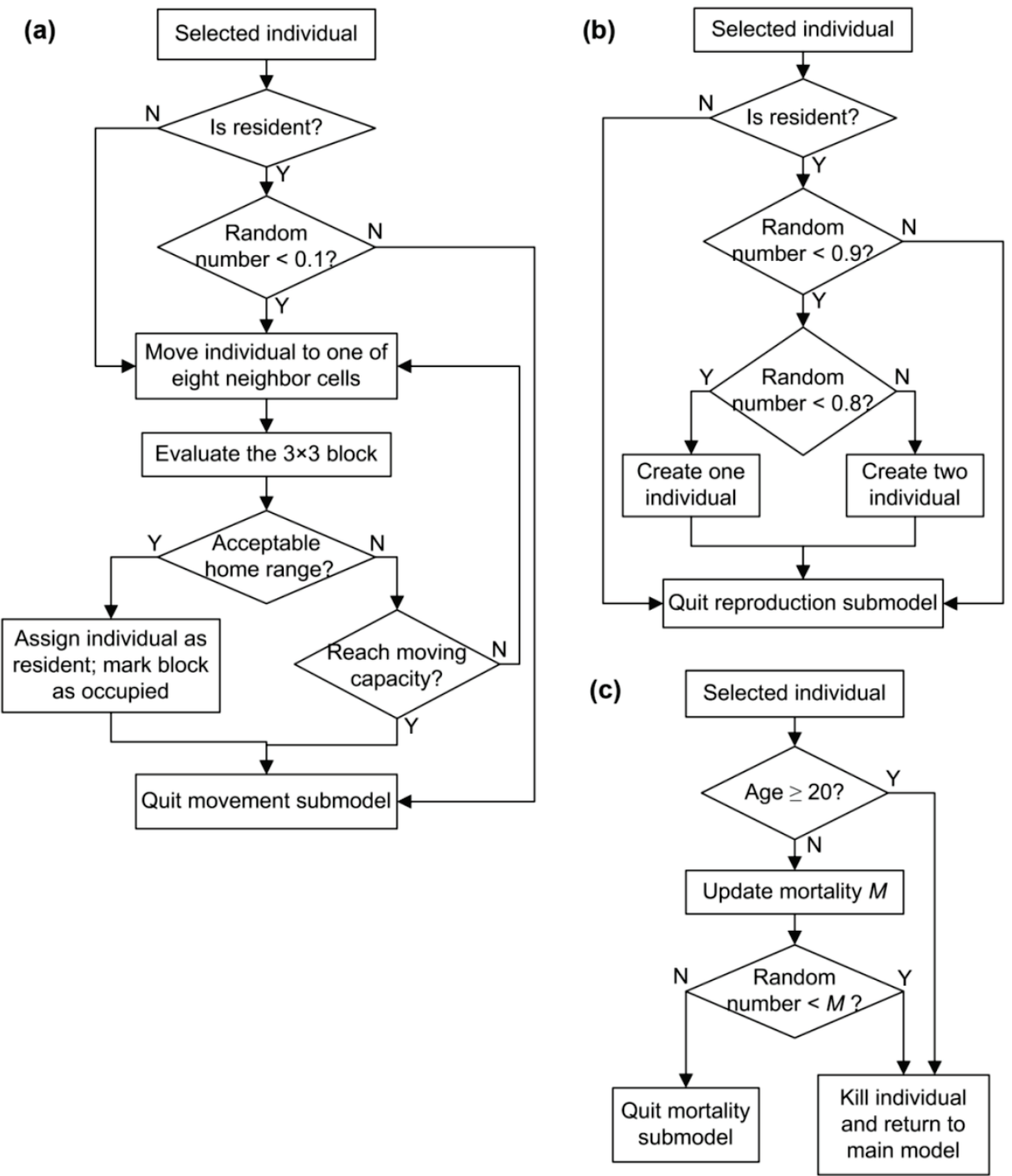

Figure A1 Diagrams of submodels for individual's reproduction, mortality, and movement within a time step: (a) movement, (b) reproduction, and (c) mortality.

Movement - We assumed that Individuals move independently on the landscape, without interacting with other individuals. Dispersing individuals (individuals in search of home range) are only allowed a limited number of spatial steps (i.e. move to neighbouring cells) in search of an acceptable home range. However, they cannot enter cells where habitat quality is lower than a threshold $\mathrm{Q}^{\prime}$ (i.e. habitat-based random walk). In addition, residents 
are assumed to abandon the occupied home range with a probability of 0.1 and start to search for a new one. The flowchart of the movement is shown in Figure A1a

Reproduction - Only residents can reproduce, with a fixed probability of 0.8 . The litter size is either one or two, with probabilities of 0.8 or 0.2 , respectively. Newborn individuals will become adults and search for their own hone range at the following time-step. The reproduction process is illustrated in Figure A1b.

Mortality - The process of mortality is shown in Figure A1c. Each individual dies according to the probability of mortality it experienced. The mortality rate $(M)$ is individual-specific, being modified by local population density and habitat quality: $M=M_{0} \cdot\left(1+\exp \left(-C \cdot(Q / J)^{2}\right)\right)$, where $M_{0}$ is the basic mortality rate, $C$ is the niche breadth coefficient of the species (Figure A2), $Q$ is the quality of the occupied cell, and $J$ is the number of individuals within the area of $5 \times 5$ cells surrounding the cell occupied by the individual. Individuals that reached the maximum lifespan die immediately.

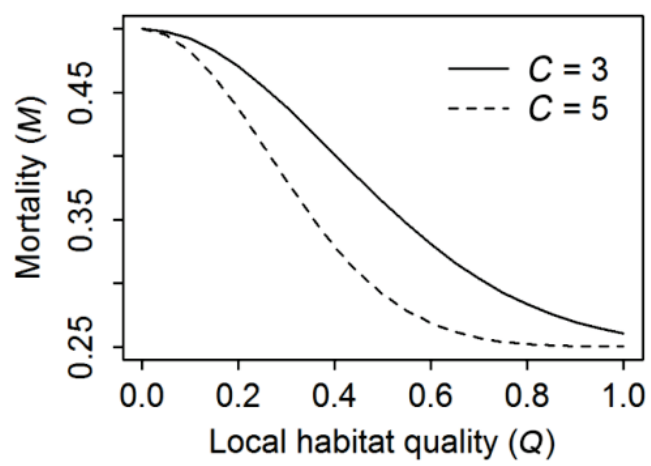

Figure A2 Simulated individual's mortality as a function of local habitat quality. $C$ is niche breadth coefficient. $C=3$ represents the specialist species; $C=5$ represents the generalist species. The basic mortality is set to 0.25 . 


\section{Appendix 2}

\section{Calculation of generalized eta-squared}

The generalized eta-squared, $\eta_{\mathrm{G}}^{2}$, is a measure of effect size that permits comparisons of results across both between-subjects and within-subjects designs (Olejnik \& Algina, 2003; Bakeman, 2005). The generalized etasquared is calculated as:

$$
\eta_{\mathrm{G}}^{2}=\frac{S S_{\text {Effect }}}{\delta \times S S_{\text {Effect }}+\sum_{\text {Measured }} S S_{\text {Measured }}+\sum_{\mathrm{K}} S S_{\mathrm{K}}}
$$

where $\delta=1$ if the effect of interest is a manipulated factor and zero otherwise. The index Measured runs over all sources of variance that do not include subjects but do involve a measured factors, (e.g., a blocking factor or a Block $\times$ Manipulated factor interaction), and $S S_{\text {Measured }}$ is the sum of squares for such an effect. The index $\mathrm{K}$ runs over all sources of variance that involve subjects or covariates, and $S S_{\mathrm{K}}$ is the sum of squares for such a source of variation. $S S_{\text {Effect }}$ is the sum of squares for the factor for which the effect size is being estimated. 


\section{Spatial pattern of habitat quality modulates population persistence in fragmented landscapes}

This chapter is based on: Ye, X., Wang, T. and Skidmore, A. (2013) Spatial pattern of habitat quality modulates population persistence in fragmented landscapes. Ecological Research, 28:949-958. 


\begin{abstract}
Habitat quality is one of the important factors determining population dynamics and persistence, yet few studies have examined the effects of spatial heterogeneity in within-patch habitat quality. In this paper, we use a spatially explicit agent-based model to investigate how habitat fragmentation and spatial pattern of within-patch habitat quality affect population dynamics and long-term persistence. We simulate three levels of habitat fragmentation (ranges from continuous to highly fragmented) and three types of spatial patterns in habitat quality within patches (i.e., negatively autocorrelated, randomly distributed, and positively autocorrelated). Hypothetical species differ in their niche specialisation. The results demonstrate explicitly that spatial pattern of within-patch habitat quality plays an important role in modulating the effects of habitat fragmentation on populations. Populations become less variable in size, and experience lower probability of extinction in landscapes with positively autocorrelated within-patch habitat quality. Specifically, specialised species are more vulnerable to habitat fragmentation, but this vulnerability is greatly mitigated by positively autocorrelated habitat quality within patches, in other words, exhibiting higher resistance to habitat fragmentation. The findings of this study suggest that managing habitat quality in existing habitat remnants is important to preserve species in habitats undergoing fragmentation, particularly for those with specialised habitat requirements.
\end{abstract}

Keywords: fragmentation, spatial heterogeneity, spatial autocorrelation, niche specialisation, habitat quality, agent-based model 


\subsection{Introduction}

Understanding the factors that influence population dynamics and persistence under scenarios of habitat fragmentation is a central question in ecology (Lawler et al., 2006; Melbourne \& Hastings, 2008). Many theoretical and empirical studies suggest that species inhabiting intact habitat will exhibit greater population persistence than in fragmented habitat, i.e., "fragmentation hypothesis" (e.g., Andrén, 1994; Fahrig, 1997; Villard et al., 1999; With \& King, 1999; Flather \& Bevers, 2002). Yet most studies were built on island biogeography and metapopulation theories and assumed that quality within or among habitats is homogeneous (Klok \& De Roos, 1998). Despite habitat quality profoundly affecting population dynamics (Murphy et al., 1990; Fahrig, 2007), within-patch quality has received little attention when assessing the effect of habitat fragmentation on species in heterogeneous landscapes.

Environmental heterogeneity, that is an uneven distribution of resources, creates variation in habitat quality (or gradients) at many scales (Shaver, 2005). Emerging empirical evidence suggests that variation in patch quality plays an important role in population dynamics and persistence (e.g., Pulliam, 1988; Runge et al., 2006). When analysing habitat quality at the patch level, habitat patches were generally "averaged out" as single data points (e.g., Fleishman et al., 2002; Fraterrigo et al., 2009). Such simplifications are convenient in dealing with landscape complexity (Talley, 2007), but may overlook the spatial variation in habitat quality within patches.

Spatial variation in the quality of habitat units can have strong effects on population density and extinction events (González-Megías et al., 2005). Some empirical studies suggest that positive spatial autocorrelation in patch quality increase species persistence (Clarke et al., 1997; Matlack \& Leu, 2007), but this is contradicted by other studies indicating that increasingly positive spatial autocorrelation may result in greater extinction risks (Johst \& Drechsler, 2003; Pike et al., 2004). Spatial variability of habitat quality can be depicted by the spatial pattern of habitat quality across landscapes, i.e., spatial autocorrelation (Dormann et al., 2007). Controlling the spatial organisation of habitat quality in fragmentation research using autocorrelation, allows an accurate understanding of species-environment relationships, and means the results are not susceptible to interactions from the multiple environmental factors affecting the empirical results cited above.

Species differ in their environmental tolerance or niche-width (Pigliucci, 2001; Kolasa \& Li, 2003). Based on niche theory, ecological specialists may prefer homogeneous habitats rather than diverse habitats, while generalists 
exhibit less sensitivity to habitat variability, exhibiting higher environmental tolerance (Ewers \& Didham, 2006). The niche-width of a species should not be neglected when understanding the species response to habitat fragmentation (Henle et al., 2004). Since habitat fragmentation increases the extinction rate of local populations (Fahrig, 2002), the spatial organisation of habitat quality within the remaining fragments influence population dynamics and long-term persistence. However, few studies have examined the important interaction of species niche, fragmentation, and the spatial organisation of habitat quality within habitat remnants.

In this paper, we investigate the relative influence of habitat fragmentation, spatial pattern of habitat quality within habitat remnants, and their interactions with species having a different niche-width. Using a spatially explicit agent-based model, we test how populations with different levels of niche specialisation respond to habitat fragmentation, and how spatial pattern of habitat quality alters population responses. The results provide new insights on preserving small populations in fragmented landscapes.

\subsection{Methods}

\subsubsection{Model description}

The model simulates the reproduction, movement, and survival of closed populations on landscapes with different levels of habitat fragmentation and spatial patterns of within-patch heterogeneity in habitat quality. Our model allows for comparison of the responses of different species to landscape heterogeneity by incorporating habitat fragmentation, within-patch variations in habitat quality, and species' niche specialisation under the concept of 'landscape population model' (Wu, 2009). Model parameters and values used in the simulations are presented in Table 1.

\subsubsection{The landscape model}

The landscape is modelled as a square $100 \times 100$ cells grid consisting of a number of suitable habitat patches within a matrix of unsuitable habitat, with a periodic boundary condition (i.e., the left and right edges and the top and bottom edges of the grid are joined together). Each cell in the grid is characterized by its habitat quality $(Q$; range $0-1)$, where habitat quality simply reflects the condition affecting an individual's reproduction and survival. We define that each "habitat" cell has a quality value in the range $(0.5,1)$, whilst each "matrix" cell's quality is in the range $(0,0.5)$. Therefore, the simulated landscape is a continuous representation of habitat quality rather than a binary map of habitat and non-habitat matrix. 
(a)

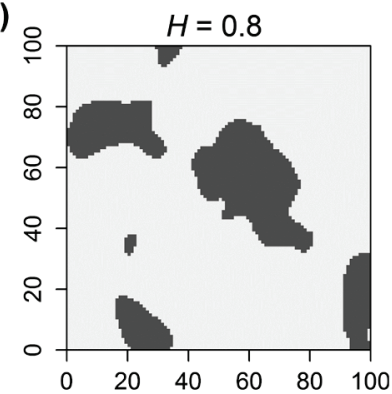

(b)

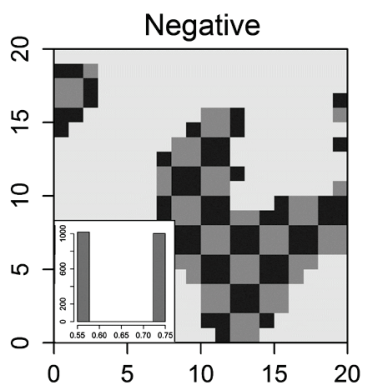

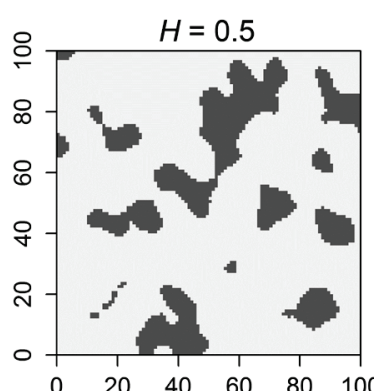
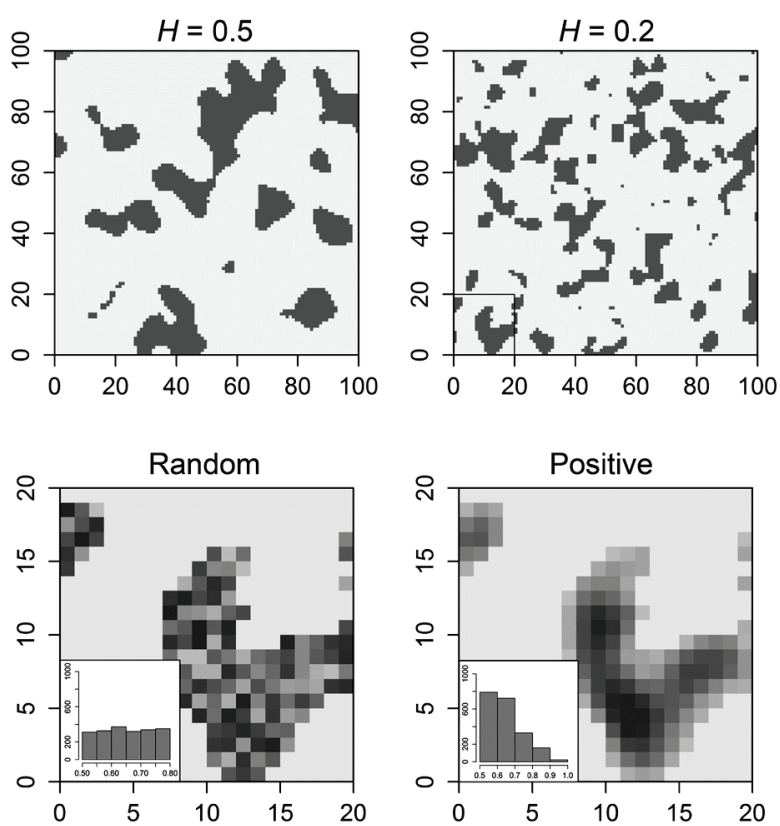

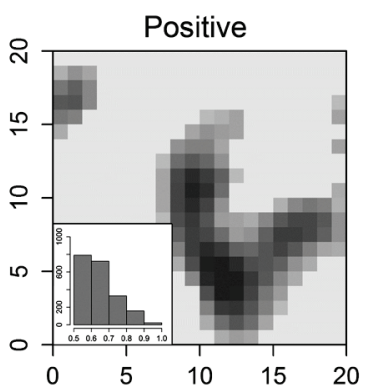

Figure 1 Snapshots of simulated landscapes differing in (a) degree of fragmentation and (b) spatial pattern of within-patch habitat quality. Darkgrey cells represent suitable habitat, while light-grey cells represent unsuitable habitat. The first row presents three levels of fragmentation: aggregated $(H=0.8)$, moderately fragmented $(H=0.5)$, and highly fragmented $(H=0.2)$; and the second row illustrates three spatial patterns of within-patch habitat quality (within lower left corner of the landscape with $H$ $=0.2$ ): negative, random, and positive autocorrelation. The small subplots in graphs of the second row are histograms showing the distribution of cell-wise habitat quality values within patches. The amount of habitat was fixed at $\mathbf{2 0}$ $\%$ of the landscape area, and the mean habitat quality of habitat cells was restricted to 0.65 .

We simulate three levels of habitat fragmentation (Figure 1a) by varying the parameter $H$ in the midpoint displacement algorithm (Saupe, 1988; Flather \& Bevers, 2002): aggregated $(H=0.8)$, moderately fragmented $(H=0.5)$, and highly fragmented $(H=0.2)$. The amount of habitat in the landscape is fixed at $20 \%$ of the total landscape area, following the theoretical predictions of fragmentation threshold affecting population (Fahrig, 1998; Flather \& Bevers, 2002).

For each landscape, we simulate three distinct spatial patterns of withinpatch habitat quality: negatively autocorrelated, randomly distributed, and positively autocorrelated (Figure $1 \mathrm{~b}$ ). In order to compare the effect of spatial patterns on populations, the mean habitat quality of habitat cells is restricted to 0.65 in all landscapes. When habitat quality is negatively autocorrelated, 
the cell quality was either 0.75 or 0.55 , with cells having the same quality value scattered throughout the habitat area. For the case of randomly distributed habitat quality, we assign each habitat cell a random value in the range $0.5-0.8$. When habitat quality is positively autocorrelated, the quality of habitat cells exhibits a decreasing gradient (in the range 0.5-1.0) from the centre to the edge of a habitat patch. Because our aim is to understand the role of spatial pattern of within-patch habitat quality, we simply assign each matrix cell a random value drawn from a normal distribution with mean 0.25 and standard deviation 0.10 in all landscapes.

\subsubsection{The population model}

We use a stochastic, single-species model to simulate the dynamics and longterm persistence of populations with limited dispersing capacity under different landscape conditions. Only females are formulated in the model, following the convention adopted in many population dynamics models (Noon \& Biles, 1990; Pulliam et al., 1992). The generations are overlapped, and no age-structure is included. Population parameters (Table 1) are adjusted arbitrarily to yield an overall population growth rate $1.05>\lambda>1$ for species with intermediate niche specialisation $\left(C_{\text {niche }}=4\right)$ under the scenario of moderate fragmentation $(H=0.5)$ and randomly distributed habitat quality within patches. We did not vary basic birth rate and basic mortality rate in the experiment, because more complex relations (e.g., including temporal stochasticity in mortality and reproduction) would by virtue of Jensen's inequality prohibit comparison between landscapes (Ruel \& Ayres, 1999; Stoddard, 2010). The time step of the model is not explicitly specified, but is assumed to be long enough for each individual to accomplish the following demographic processes: movement, reproduction, and mortality. At each time step, mortality and reproduction processes take place concurrently, after the process of movement. All demographic processes are implemented based on probabilities.

In the model, we define an adult individual that occupies a habitat cell alone as the "resident". If two or more individuals share a habitat cell, only one is randomly chosen and labelled as resident. Only resident individuals can reproduce, giving birth to one offspring with a probability of $B=B_{\text {basic }} \times Q$, where $B_{\text {basic }}$ being the basic birth rate, and $Q$ being the actual habitat quality of the occupied cell. New-born individuals are attached to their mothers, and become adults being able to search for suitable habitat cells at the following time step.

Only "non-resident" individuals are involved in the movement process, i.e., moving around in search of unoccupied habitat cells. At each time step, "nonresident" individuals move in a cell-by-cell random walk mode (i.e., move to 
one of the 8 neighbouring cells each movement step), and continued until reaching an unoccupied habitat cell or a predefined maximum movement steps, $D_{\max }$. The assumption of random walk seems to be unrealistic for species with active dispersal, but is conservative in the sense that effects of fragmentation are easy to detect (Fahrig, 1998). Dispersal risk is considered by incorporating a per-step probability of death into the process of movement (Dal Forno \& Merlone, 2004). Therefore, the chance of survival of an individual after moving $D$ steps $\left(D \leq D_{\max }\right)$ is given by the function: $M_{\text {dispersal }}$ $=\left(1-M_{\text {per-step }}\right)^{D}$, where $M_{\text {per-step }}$ is the mortality rate per movement step. To make model analyses feasible, we assume that individuals' movements are independent of each other without competing for space. Individuals that fail to find acceptable habitat cells, if survived, will continue searching in the next time step.

Table 1 Model parameters and values used in the simulations.

\begin{tabular}{lll}
\hline Parameter & Notation & Values \\
\hline Landscape & & \\
$\quad$ Landscape size & & $100 \times 100$ cells \\
Amount of suitable habitat in the landscape & & $20 \%$ \\
Degree of habitat fragmentation & $H$ & $0.8,0.5,0.2$ \\
Range of habitat quality & $Q$ & $0-1$ \\
Cut-off quality value for habitat and matrix & & 0.5 \\
Mean habitat quality of habitat cells & & 0.65 \\
Mean habitat quality of matrix cells & & 0.25 \\
Population & & 20 time steps \\
Maximum life span & $B_{\text {basic }}$ & 0.6 \\
Basic reproduction probability & $M_{\text {basic }}$ & 0.25 \\
Basic habitat-dependent mortality rate & $M_{\text {per-step }}$ & 0.01 \\
Mortality rate per movement step & $D_{\text {max }}$ & 10 cells \\
Maximum movement steps per time step & $C_{\text {niche }}$ & $3,4,5$ \\
Niche-width coefficient & & \\
Model simulation & & 200 \\
Starting number of individuals & 300 \\
Maximum time steps in simulation &
\end{tabular}

Each time step, individual die or survive with a habitat-dependent mortality rate $\left(M_{\text {quality }}\right)$, except for those reached the maximum lifespan (which die immediately). The mortality rate is individual-specific, depending on the habitat quality and crowding of the occupied cell: $M_{\text {quality }}=M_{\text {basic }} \times(1+\exp$ $\left.\left(-\left(C_{\text {niche }} \times Q / N_{\text {share }}\right)^{2}\right)\right)$, where $M_{\text {basic }}$ is the basic mortality rate, $C_{\text {niche }}$ is the coefficient defining species niche specialisation, $Q$ is the actual habitat quality of the occupied cell, and $N_{\text {share }}$ is the number of individuals within the 
occupied cell. When an individual is subjected to a lower habitat quality or a crowed condition, its mortality rate tends to be much higher that others (Figure 2a). Populations with a smaller value of $C_{\text {niche }}$ would be more sensitive to changes in habitat quality (Figure $2 b$ ), indicating a more specialised species.
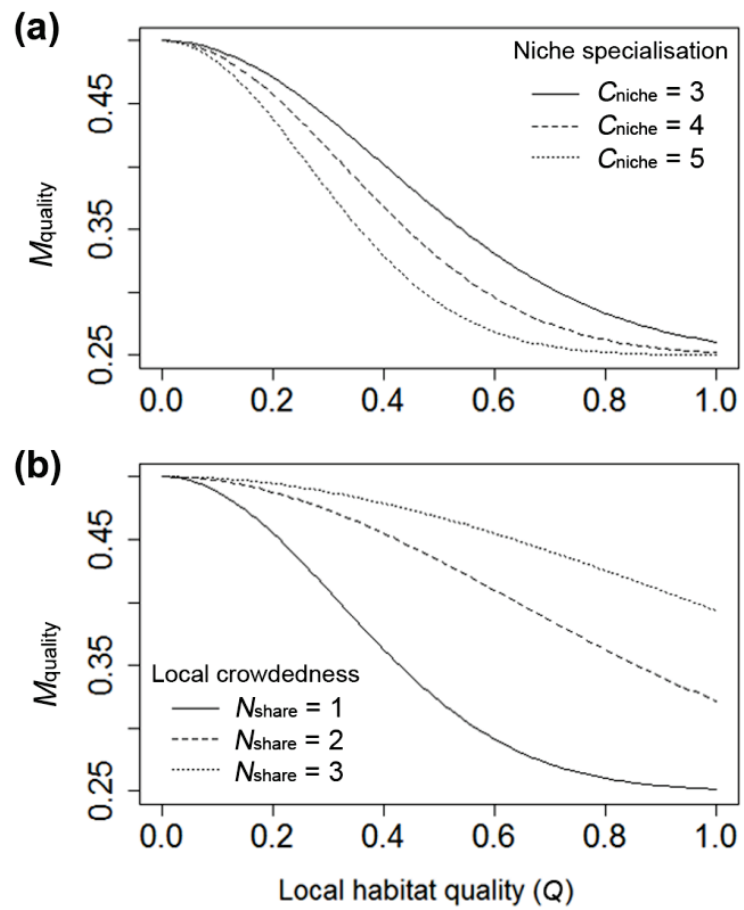

Figure 2 Changes in habitat-dependent mortality rate as (a) niche specialisation and (b) local crowdedness vary. Symbol " $C_{\text {niche" }}$ in panel (a) refers to the coefficient adjusting the level of niche specialisation; and $C_{\text {niche }}=$ 3,4 , and 5 represent high, moderate, and low level of species specialisation, respectively. Symbol " $N_{\text {share }}$ " in panel (b) refers to local crowdedness which is measured as the number of individuals within an occupied cell. $C_{\text {niche }}=\mathbf{5}$ was used in the evaluation of mortality rate responding to local crowdedness.

\subsubsection{Model implementation}

The combination of habitat fragmentation and spatial organisation of habitat quality within patches resulted in 9 landscape scenarios $(3 \times 3=9)$ in the experiment.

We designed three levels of niche specialisation $\left(C_{\text {niche }}\right)$ to assess potential interactions between spatial pattern of landscape heterogeneity and species niche specialisation, therefore resulting 27 unique treatments (9 landscape 
scenarios $\times 3$ levels of $\left.C_{\text {niche }}\right)$. For each treatment, 100 replicates were conducted in order to minimise stochasticity effects during simulation. At the beginning of each replicate, 200 individuals were randomly distributed within habitat area in the landscape. The model continued until the population went extinct or 300 time steps were counted. Preliminary tests indicated that the length of 300 time steps was sufficient for either a dynamic equilibrium or extinction to be achieved (data not shown). For populations persisting to the end of the run, population size was recorded as the mean over the last 50 time steps. We also recorded mean mortality rate of individuals and mean habitat quality of occupied cells for better understanding of underlying mechanisms involved in the regulation of population dynamics over the last 50 time steps. The simulations were implemented in the NetLogo 4.1.2 (Wilensky, 1999), a friendly environment for spatially explicit agent-based modelling.

\subsubsection{Statistical analysis}

Our analysis focused on responses of the population size and persistence probability. Persistence probability was calculated as the proportion of the populations persisting to the end of the simulation over 100 replicate runs. Coefficient of variation ( $\mathrm{CV}$; calculated as the ratio of standard deviation to mean) was used to measure the degree of temporal variation in population size. Prior to analysis, population size data were transformed using $\log (x+1)$ to increase data normality. We performed Kolmogorov-Smirnov one-sample test (K-S test) and found that for each response variables, the data were normally distributed in most treatments. We used a factorial ANOVA on general linear models to test the main effects of all factors and their interactions. All factors were treated as categorical variables in accordance with the experimental design. Percentage of variance explained (Cohen, 1988) and generalized eta-squared (Olejnik \& Algina, 2003; Bakeman, 2005) were used to measure the importance of the effect of the factors and their interactions on response variables. All statistical analyses were conducted using R software, version 2.13.0 (R Development Core Team, 2011) and the 'ez' package (http://cran.rproject.org/web/packages/ez).

\subsection{Results}

Overall, habitat fragmentation caused substantial declines in both population size and long-term persistence of the hypothetical species (Figure 3), and this impact varied significantly with species' niche specialisation and spatial pattern of within-patch habitat quality. For example, species with narrow niche-width $\left(C_{\text {niche }}=3\right)$ was the most vulnerable to habitat fragmentation when habitat quality was negatively autocorrelated or in a random fashion ("Negative" and "Random" in Figure 3a and 3b), and became extinct when 
inhabiting highly fragmented habitats $(H=0.2)$. In contrast, positively autocorrelated habitat quality greatly buffered the negative effects of habitat fragmentation, resulting in gradual decreases in both population size and long-term persistence ("Positive" in Figure $3 a$ and 3b). As we expected, habitat fragmentation had the strongest effect on populations, accounting for $\sim 32 \%$ of the variation in population size (" $N$ " in Table 2). Species niche specialisation and spatial pattern of habitat quality explained $\sim 25 \%$ and $\sim 13 \%$, respectively, of the variation in population size (" $N "$ in Table 2 ). Their interactions also had significant but relatively small effects on population size ("N" in Table 2).

Table 2 Summary of proportions of variation in response variables explained by three factors and their interactions in ANOVAs. Factor "Frag" refers to habitat fragmentation; factor "SAC" refers to spatial pattern of habitat quality within patches; and factor "Niche" refers to species' niche-width. The response variables are: $N=$ population size, $C V=$ temporal variation in population size, $M=$ mean mortality rate of individuals, $Q_{\text {occupied }}=$ mean habitat quality of cells occupied by individuals.

\begin{tabular}{lrrrr}
\hline \multirow{2}{*}{ Source of variation } & \multicolumn{4}{c}{ Explained variation in response variable (\%) } \\
\cline { 2 - 5 } & $\mathrm{N}$ & $\mathrm{CV}$ & $\mathrm{M}$ & Q $_{\text {occupied }}$ \\
\hline Frag & 31.85 & 22.79 & 3.07 & 1.39 \\
SAC & 13.09 & 10.16 & 33.78 & 52.66 \\
Niche & 25.48 & 20.2 & 52.21 & 1.08 \\
Frag $\times$ SAC & 0.2 & 1.17 & 0.6 & 0.64 \\
Frag $\times$ Niche & 1.33 & 1.41 & 1.09 & 0.18 \\
SAC $\times$ Niche & 2.06 & 2.88 & 2.0 & 0.71 \\
Frag $\times$ SAC $\times$ Niche & 1.41 & 1.64 & 0.03 & 0.53 \\
\hline
\end{tabular}

Note: Detailed factorial ANOVAs are presented in Appendix 1. 
(a)

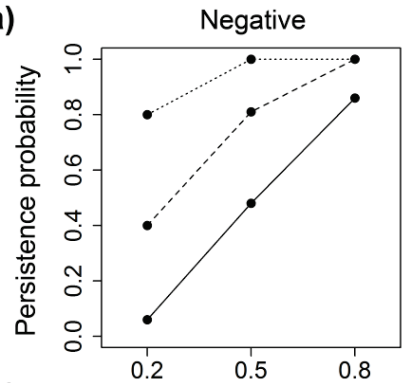

(b)

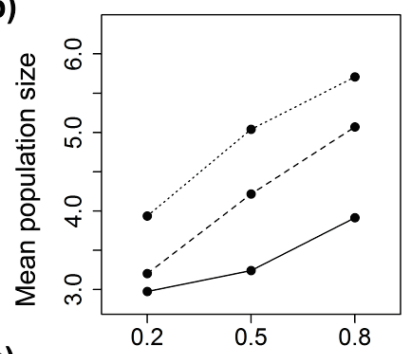

(c)

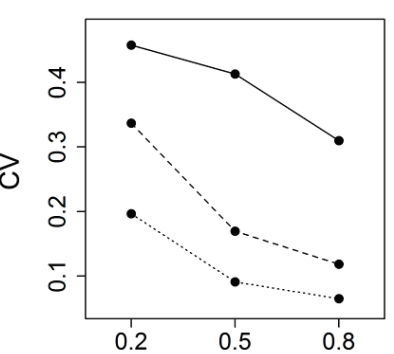

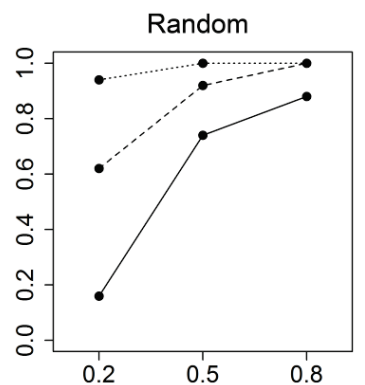
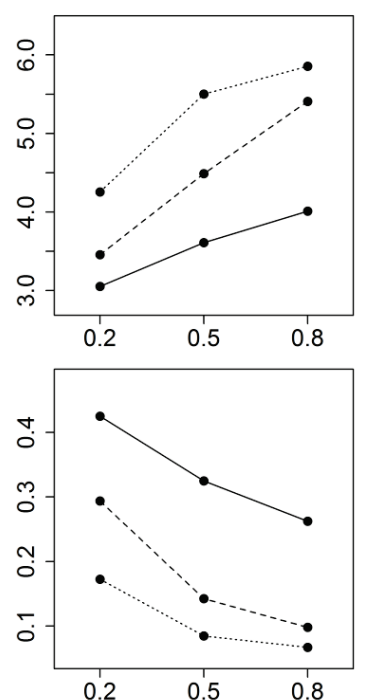

Degree of habitat fragmentation $(H)$
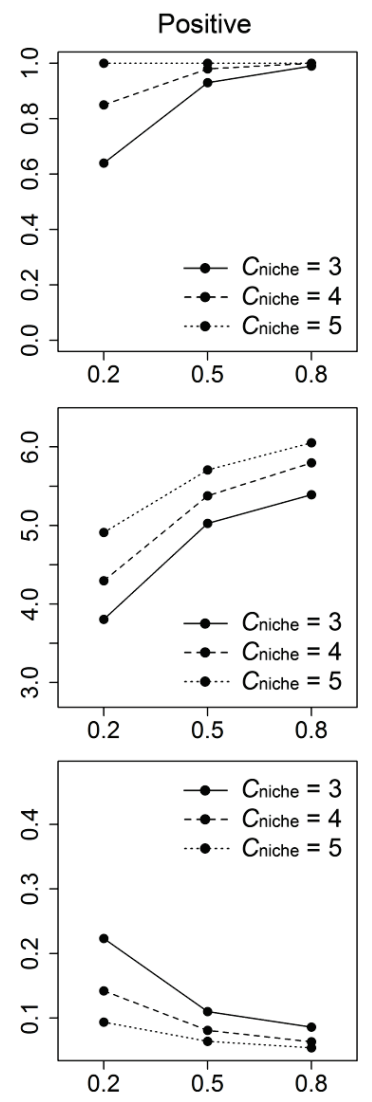

Figure 3 Responses of populations varying in niche specialisation to habitat fragmentation and spatial pattern of habitat quality within patches: (a) probability of persistence, (b) mean population size, and (c) temporal variation in population size. Mean population sizes were plotted in log scale, and the standard errors were not displayed due to their small sizes. Columns ("Negative", "Random", and "Positive") represent different spatial patterns of habitat quality within patches. $C_{\text {niche }}=3,4$, and 5 represent high, moderate, and low level of species' niche specialisation, respectively.

Similarly, habitat fragmentation had the strongest effect on temporal variation $(\mathrm{CV})$ in population size, followed by species niche specialisation and spatial pattern of habitat quality within patches ("CV" in Table 2). Increasing habitat fragmentation also increased the CV of population size (Figure 3c). Moreover, populations of more specialised species exhibited higher CVs in population size, especially when inhabiting landscapes with negatively autocorrelated or randomly distributed habitat quality ("Negative" and "Random" in Figure 3c). 
(a)

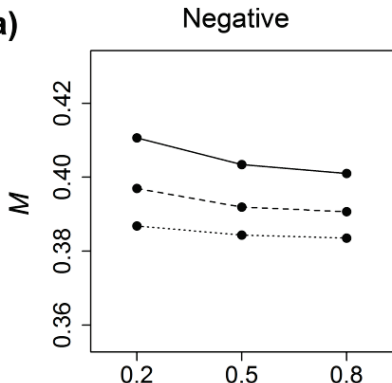

(b)

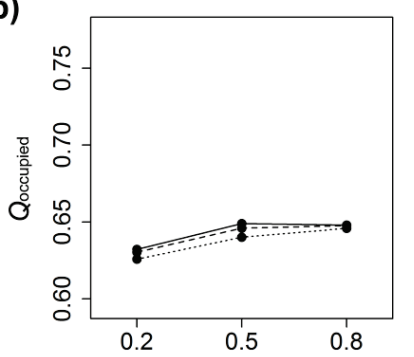

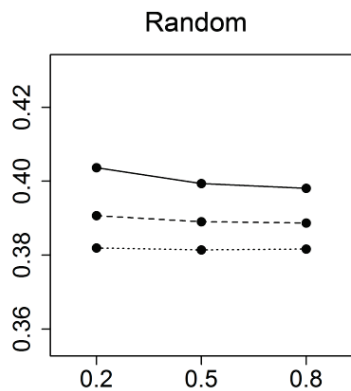

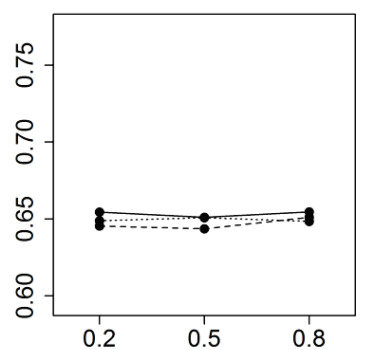

Degree of habitat fragmentation $(H)$
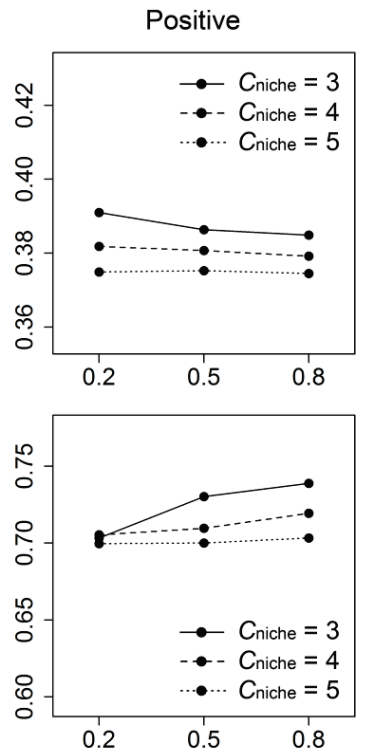

Figure 4 Changes in (a) mean mortality rate of individuals and (b) mean habitat quality of cells occupied by individuals varying in niche specialisation and subjected to different levels of habitat fragmentation and spatial pattern of habitat quality within patches. $C_{\text {niche }}=3,4$, and 5 represent high, moderate, and low level of species specialisation, respectively. Columns ("Negative", "Random", and "Positive") represent different spatial pattern of habitat quality within patches.

The mean mortality rate of individuals was negatively correlated with species niche-width, whilst populations generally experienced lower mortality rate when habitat quality was positively autocorrelated (Figure 4a). Species niche specialisation and spatial pattern of habitat quality both had strong effects, explained $\sim 52 \%$ and $\sim 34 \%$, respectively, of the variation in mean mortality rate of individuals (" $M$ " in Table 2 ). The main effect of habitat fragmentation was also significant, but accounted only for $\sim 3 \%$ of the variation in mean mortality rate of individuals (" $M$ " in Table 2).

For the mean habitat quality of cells occupied, spatial pattern of habitat quality had the strongest effect and accounted for $~ 53 \%$ of the variation in this response variable ("Q $Q_{\text {occupied }}$ " in Table 2 ). The mean habitat quality of cells occupied was the highest when habitat quality was positively autocorrelated, indicating that individuals were confined to cells with higher habitat quality (Figure 4b). In addition, the mean habitat quality of cells occupied for specialised species was generally higher; this may be partly because the specialised species had a lower population size than other species. Habitat fragmentation and niche specialisation both had little effects, each accounting 
only for $\sim 1 \%$ of the variation in mean habitat quality of cells occupied by individuals ("Qoccupied" in Table 2).

\subsection{Discussion}

In this study we explored the effects of habitat fragmentation and withinpatch habitat quality using simulated populations. Consistent with previous research (e.g., With \& King, 1999; Fahrig, 2002; Wiegand et al., 2005), our model suggested that habitat fragmentation was the most important factor governing population size and long-term persistence. However, our results showed explicitly that spatial pattern of within-patch habitat quality played an important role in modulating the effects of habitat fragmentation on populations. In the case of landscapes with positively autocorrelated withinpatch habitat quality, populations became less variable in size and experienced lower probability of extinction. Specifically, populations of specialised species were more vulnerable to habitat fragmentation, but this vulnerability was greatly mitigated by positively autocorrelated habitat quality within patches.

It is not surprising that spatial pattern in habitat quality was important to populations inhabiting a heterogeneous landscape. Given a limited movement capacity, individuals inhabiting landscapes with positively autocorrelated habitat quality are likely to encounter high-quality cells, hence, increasing the fitness of individuals as well as reducing the probability of mortality. In contrast, habitat quality in a regular pattern (negatively autocorrelated in space) results in substantial contrast between adjacent sites at a fine scale, thereby decreasing the probability of finding high-quality cells. For example, when within-patch habitat quality was positively autocorrelated, the mean habitat quality of cells occupied was the highest, indicating that individuals were confined to cells with higher habitat quality, especially for populations of specialised species. These results point to the importance of managing spatial structure within habitats in the face of increasing habitat fragmentation. For species experiencing ongoing habitat fragmentation, from the conservation point of view, attention should focus on managing habitat quality in existing habitat remnants as well as the implementation of an optimal patch spatial structure.

The findings of this study also reflect the limitations imposed on populations by their niche specialisation interacting with habitat fragmentation and spatial pattern of habitat quality. Increasing in species' niche-width in our model greatly elevated species' population size and long-term persistence on landscapes with negatively autocorrelated or randomly distributed habitat quality. In general, a population with a narrow niche-width has a relative low probability of survival, and consequently, its persistence depends heavily on 
the chance of individuals to find suitable habitats (Fraterrigo et al., 2009). However, habitat fragmentation can restrict the spread of organisms, particularly for those with a limited movement capacity (Muller et al., 2004; Wiegand et al., 2005), increasing the risk of local extinction. Furthermore, habitat fragmentation forces individuals to spend more time in unsuitable habitat where reproduction is not possible or the rate of mortality is higher (Fahrig, 2002), leading to a greater loss of individuals from existing populations. From a different perspective, when a species' dispersal capacity is limited relative to the spatial distribution of the habitat patches it can occupy, a metapopulation structure can emerge, where local populations within habitat patches are connected by infrequent dispersal among patches (Hanski, 1999). Negatively autocorrelated or randomly distributed withinpatch habitat quality, if present, could amplify the joint effects of habitat fragmentation and demographic stochasticity by decreasing the probability of population recovery from local extinction.

Our experiment further provides additional evidence that fine-scale variation within habitats at which individual animals live is critical for understanding population dynamics in fragmented landscapes (Wiens et al., 1993b; Flather \& Bevers, 2002; Matlack \& Leu, 2007; Ye et al., 2013a). Habitat fragmentation results in separation of habitat patches as well as a decrease in patch area, which can reduce the size of local populations and the likelihood of individual transfer between patches (Hanski, 1991). From an ecological perspective, small populations are less resistant to environmental stochasticity and more likely to go extinct (Levin, 2009). The interplay between species traits and fine-scale quality variation can cause complex responses of population to habitat fragmentation. Furthermore, spatial pattern of habitat quality within patches had a much stronger impact on specialised species, indicating that specialist species may be susceptible to local variability within habitats (Kindvall, 1996; Lloyd, 2008). Habitat quality may be more relevant than patch connectivity in the population dynamics of habitat specialists, but the degree of association for each factor may vary under different scenarios.

Our model was simplified in order to generalise population response to habitat fragmentation, and therefore several effects could be studied in further detail. For example, male individuals were excluded in the model based on the notion that females are the reproductive unit that determine population dynamics (Pulliam et al., 1992; Wiegand et al., 1999). We did not examine the potential effect of dispersal distance in the model despite recent research suggesting that increasing dispersal distance may promote the persistence of populations in fragmented landscapes (e.g., Thomas, 2000; Johst et al., 2002; Reed, 2004). Under the assumption of random walk, however, species with 'intermediate' dispersal abilities would be most 
sensitive to habitat fragmentation, largely due to intermediate mobility resulted in individuals leaving one fragment but failing to reach the next, in turn leading to an overall increase in mortality (Fahrig, 1998; Wiegand et al., 1999; Thomas, 2000). Such differences imply that one should carefully consider the influence of species traits when interpreting the effect of habitat fragmentation (Diffendorfer et al., 1995; Stouffer \& Bierregaard, 1995). Moreover, spatiotemporal fluctuation in environmental conditions (e.g., environmental stochasticity) could also be an important factor in determining the continued survival of small populations in face of habitat fragmentation (Stacey \& Taper, 1992; Fujiwara, 2009). There remains a need to understand the joint effects of environmental spatial variability and temporal stochasticity on fragmented populations in heterogeneous landscapes, though were beyond the scope of the study presented here.

\section{Acknowledgements}

This work was supported by the Erasmus Mundus External Co-operation Window (EMEWC) Programme of the European Union and co-funded by the ITC Research Fund. 


\section{Appendix 1}

Table A1 Factorial ANOVAs on population size and temporal variation in population size (CV) of species varying in niche-width and subjected to habitat fragmentation and spatial variation in habitat quality within patches. Factor "Frag" refers to habitat fragmentation; factor "SAC" refers to spatial pattern of habitat quality within patches; and factor "Niche" refers to species" niche-width.

\begin{tabular}{|c|c|c|c|c|c|}
\hline Source of variation & $d f$ & $F$ & $P$ & $\%$ of Total SS & ges \\
\hline \multicolumn{6}{|l|}{ Mean population size } \\
\hline Frag & 2 & 857.17 & $<0.001$ & 31.85 & 0.56 \\
\hline SAC & 2 & 352.36 & $<0.001$ & 13.09 & 0.35 \\
\hline Niche & 2 & 685.82 & $<0.001$ & 25.48 & 0.51 \\
\hline Frag $\times \mathrm{SAC}$ & 4 & 2.73 & 0.028 & 0.20 & 0.01 \\
\hline Frag $\times$ Niche & 4 & 17.92 & $<0.001$ & 1.33 & 0.05 \\
\hline SAC $\times$ Niche & 4 & 27.66 & $<0.001$ & 2.06 & 0.08 \\
\hline Frag $\times$ SAC $\times$ Niche & 8 & 9.49 & $<0.001$ & 1.41 & 0.05 \\
\hline \multicolumn{6}{|c|}{ Temporal variation in population size (CV) } \\
\hline Frag & 2 & 379.23 & $<0.001$ & 22.79 & 0.36 \\
\hline SAC & 2 & 169.11 & $<0.001$ & 10.16 & 0.21 \\
\hline Niche & 2 & 336.15 & $<0.001$ & 20.20 & 0.34 \\
\hline Frag $\times$ SAC & 4 & 9.71 & $<0.001$ & 1.17 & 0.03 \\
\hline Frag $\times$ Niche & 4 & 11.71 & $<0.001$ & 1.41 & 0.03 \\
\hline SAC $\times$ Niche & 4 & 23.97 & $<0.001$ & 2.88 & 0.07 \\
\hline Frag $\times$ SAC $\times$ Niche & 8 & 6.81 & $<0.001$ & 1.64 & 0.04 \\
\hline
\end{tabular}

Note: "ges" is the generalized eta-squared. 
Table A2 Factorial ANOVAs on mean mortality rate of individuals and mean habitat quality of cells occupied by populations varying in niche-width and subjected to habitat fragmentation and spatial variation in habitat quality within patches. Factor "Frag" refers to habitat fragmentation; factor "SAC" refers to spatial pattern of habitat quality within patches; and factor "Niche" refers to species' niche-width.

\begin{tabular}{|c|c|c|c|c|c|}
\hline Source of variation & $d f$ & $F$ & $P$ & $\%$ of Total SS & ges \\
\hline \multicolumn{6}{|c|}{ Mean mortality rate of individuals (M) } \\
\hline Frag & 2 & 281.11 & $<0.001$ & 3.07 & 0.30 \\
\hline SAC & 2 & 3090.54 & $<0.001$ & 33.78 & 0.82 \\
\hline Niche & 2 & 4776.91 & $<0.001$ & 52.21 & 0.88 \\
\hline Frag $\times$ SAC & 4 & 27.23 & $<0.001$ & 0.59 & 0.08 \\
\hline Frag $\times$ Niche & 4 & 49.85 & $<0.001$ & 1.09 & 0.13 \\
\hline SAC $\times$ Niche & 4 & 91.39 & $<0.001$ & 1.99 & 0.22 \\
\hline Frag $\times$ SAC $\times$ Niche & 8 & 0.59 & 0.788 & $<0.01$ & $<0.01$ \\
\hline \multicolumn{6}{|c|}{ Mean habitat quality of cells occupied (Qoccupied) } \\
\hline Frag & 2 & 21.41 & $<0.001$ & 1.39 & 0.03 \\
\hline SAC & 2 & 813.36 & $<0.001$ & 52.66 & 0.55 \\
\hline Niche & 2 & 16.61 & $<0.001$ & 1.08 & 0.03 \\
\hline Frag $\times$ SAC & 4 & 4.93 & 0.002 & 0.64 & 0.02 \\
\hline Frag $\times$ Niche & 4 & 1.36 & 0.244 & 0.18 & $<0.01$ \\
\hline SAC $\times$ Niche & 4 & 5.49 & 0.001 & 0.71 & 0.02 \\
\hline Frag $\times$ SAC $\times$ Niche & 8 & 2.04 & 0.039 & 0.53 & 0.01 \\
\hline
\end{tabular}

Note: "ges" is the generalized eta-squared. 


\section{Joint effects of habitat heterogeneity and species' life-history traits on population dynamics in spatially structured landscapes}

This chapter is based on: Ye, X., Skidmore, A. and Wang, T. (2014) Joint effects of habitat heterogeneity and species' life-history traits on population dynamics in spatially structured landscapes. PLOS ONE, 9:e107742. 


\section{Abstract}

Both habitat heterogeneity and species' life-history traits play important roles in driving population dynamics, yet there is little scientific consensus around the combined effect of these two factors on populations in complex landscapes. Using a spatially explicit agent-based model, we explored how interactions between habitat spatial structure (defined here as the scale of spatial autocorrelation in habitat quality) and species life-history strategies (defined here by species environmental tolerance and movement capacity) affect population dynamics in spatially heterogeneous landscapes. We compared the responses of four hypothetical species with different life-history traits to four landscape scenarios differing in the scale of spatial autocorrelation in habitat quality. Our results showed that the population size of all hypothetical species exhibited a substantial increase as the scale of spatial autocorrelation in habitat quality increased, yet the pattern of population increase was shaped by species' movement capacity. The increasing scale of spatial autocorrelation in habitat quality promoted the resource share of individuals, but had little effect on the mean mortality rate of individuals. Species' movement capacity also determined the proportion of individuals in high-quality cells as well as the proportion of individuals experiencing competition in response to increased spatial autocorrelation in habitat quality. Positive correlations between the resource share of individuals and the proportion of individuals experiencing competition indicate that large-scale spatial autocorrelation in habitat quality may mask the density-dependent effect on populations through increasing the resource share of individuals, especially for species with low mobility. These findings suggest that low-mobility species may be more sensitive to habitat spatial heterogeneity in spatially structured landscapes. In addition, localized movement in combination with spatial autocorrelation may increase the population size, despite increased density effects.

Keywords: spatial autocorrelation, habitat quality, environmental tolerance, movement limitations, population dynamics, agent-based model 


\subsection{Introduction}

Habitat heterogeneity is increasingly recognized as a significant factor affecting the dynamics and persistence of populations (Bolker, 2003; Pike et al., 2004; Lovett et al., 2005; Lou, 2006; Wu \& Hobbs, 2007). For mobile species inhabiting complex landscapes, spatial heterogeneity can influence their behaviour, thereby affecting survival and reproductive performance (Kendall \& Fox, 1998; Zając et al., 2008; Burgess et al., 2011). Most frequently, the effect of spatial heterogeneity has been studied either under a so-called binary 'habitat-matrix' framework (Pulliam, 1988; Pulliam et al., 1992; Fleishman et al., 2002) or under the assumption of stochastic variability wherein local variation is random in space (Kendall \& Fox, 1998; Lou, 2006). However, defining a landscape as discrete habitat/matrix patches overlooks the spatial pattern of continuously varying habitat quality that might facilitate or constrain the performance of individuals and therefore population dynamics (Wiegand et al., 1999; Humphries et al., 2013). In addition, predictions of population dynamics are also influenced by how the spatial variation in habitat quality within a landscape is calculated (Ye et al., 2013b). In this sense, a continuous representation of habitat quality is expected to more accurately reflect how a species experiences a heterogeneous landscape (Stoddard, 2010).

One general property of landscape heterogeneity of particular importance for ecological dynamics is its spatial autocorrelation structure, which can be defined as the property of random variables (e.g., habitat quality) taking values over distance that are more (or less) similar than expected for randomly associated pairs of observations due to geographic proximity (Dutilleul \& Legendre, 1993; Legendre, 1993; Harrison \& Bruna, 1999). The spatial autocorrelation in habitat quality is known to affect species persistence, for instance, increased spatial autocorrelation could reduce the persistence of small populations (Clarke et al., 1997; Petchey et al., 1997; Schiegg, 2003). Yet the effect of habitat spatial structure on population dynamics has rarely been tested for species in spatially structured landscapes. Bolker (2003) incorporated spatially correlated heterogeneity into simulation models of sessile organisms and found that spatial autocorrelation generally improves population viability. For mobile species, however, the performance of a population may become different if spatial autocorrelation occurs within the range of dispersal (Schooley \& Branch, 2007; Jacobson \& Peres-Neto, 2010). From an ecological standpoint, the effects of habitat spatial structure on mobile species depend not only on the scale of spatial autocorrelation, but also on how this scale relates to the species movement capacity and habitat requirements (Lovett et al., 2005; Jacobson \& Peres-Neto, 2010). If the scale of spatial autocorrelation is low, the likelihood of an individual encountering a different environment will 
increase quickly as it moves away from its present location (Schooley \& Branch, 2007). If the movement range is large, the population becomes wellmixed and hence spatially unstructured (Brachet et al., 1999; Bowler \& Benton, 2005). Moreover, species differ in their environmental tolerance, thereby exhibiting differential responses to habitat conditions (Jonsen \& Fahrig, 1997; Pigliucci, 2001; Kolasa \& Li, 2003). Therefore, we anticipate that habitat spatial structure will interact with species' environmental tolerance and movement capacity in determining species' demographic performance as well as population dynamics (Pulliam \& Danielson, 1991; Mortelliti et al., 2010). For instance, spatial autocorrelation in habitat quality may lead to spatial aggregation of individuals, thereby affecting local population density or competitive pressure on individuals (Dhondt et al., 1992; Rodenhouse et al., 1997; Boeye et al., 2014). Under such circumstances, individual-level variation can also provide underlying mechanisms of population regulation (Jager, 2001). However, analytical models do not take into account the complexity of the multiple concurrent interactions (e.g., competition between individuals) which may influence population dynamics (Parrott \& Kok, 2000; Getz \& Saltz, 2008). The key to improve understanding of the role of habitat spatial structure in driving the population dynamics of mobile species is therefore to link individual behaviours to population phenomena in a spatially explicit modelling framework (Wiens et al., 1993a; Wiegand et al., 1999; Wu \& Hobbs, 2002).

In this paper, we explored how habitat spatial structure interact with species' environmental tolerance and movement capacity to affect the population dynamics of mobile species using a spatially explicit agent-based modelling approach. We compared the responses of four virtual species to four landscape scenarios differing in the scale of spatial autocorrelation in habitat quality. The ecological motivation for this simulation was to understand the combined impacts of these factors on mobile species that are dispersal limited and experience habitat heterogeneity (e.g., animals in mountainous forests). We hypothesized that for species in landscapes with small-scale spatial autocorrelation, the movement-distance does not have a strong effect on population dynamics, whereas for species in landscapes with large-scale spatial autocorrelation, short distance movement would increase the viability of the population.

\subsection{Methods}

\subsubsection{The agent-based model}

The present spatially explicit, agent-based model simulates the population dynamics of a single virtual species in a spatially structured landscape. The description of the model follows the ODD protocol (overview, design 
concepts, and details) for describing individual- and agent-based models proposed by Grimm et al. (2006a; 2010). The model was implemented in the $\mathrm{R}$ environment ( $\mathrm{R}$ Development Core Team, 2011) with the package 'gstat' (Itakura et al., 2010).

\subsubsection{Purpose}

The purpose of this model is (i) to understand how habitat spatial structure (depicted by the scale of spatial autocorrelation in habitat quality) influences the population dynamics of mobile species, and (ii) to explore how species' life-history traits shape the population responses in relation to habitat spatial structure. Since this is a purely theoretical study, the model simulates population performance of four hypothetical species that differ in aspects of life-history traits (low-tolerance vs. high-tolerance; low-mobility vs. highmobility), rather than predict the population dynamics of any specific species. In other words, the model's formulation tends to increase generality in a trade-off for realism and precision.

\subsubsection{Entities, state variables, and scales}

The model contains two types of entities: individuals and landscape. The landscape is a grid of $30 \times 30$ habitat cells, in which each cell represents a discrete space of the environment, and is characterised by spatial coordinates and habitat quality ( $Q$; range $0-1$ ), where habitat quality simply reflects resource conditions essential for an individual's performance. Cell size is not spatially specified, but is assumed to fulfil the space requirement of an individual. The simulated landscape is, therefore, a continuous representation of habitat quality rather than a binary mosaic of habitat and matrix. The individuals are characterized by the following state variables: identity number, age, spatial location (cell coordinates), resource share, reproduction rate, mortality rate, and moving distance per time step. Only females are simulated in the model, a strategy common to many population models (Pulliam et al., 1992; Wiegand et al., 1999). No age-structure is included in the model. Values of the demographic parameters (see Table 1) are selected in order to meet reasonable assumptions about the hypothetical species (i.e. long-lived animals which generally have low mortality rate and high probability of reproductive success), thereby ensuring this generic model to generate plausible projections. Time runs in discrete steps and the simulation lasts 100 time-steps. The length of a time step is not explicitly specified, but is assumed to be long enough for each individual to accomplish all demographic processes (i.e., movement, reproduction, and mortality). 


\subsubsection{Process overview and scheduling}

Individuals may move, reproduce, and/or die at each time step. The order of individuals is randomized per time step, and the order of the three events (i.e. move, reproduce, and/or die) is randomized for each individual per time step. Probabilities of mortality, reproduction, and movement of an individual per time step depend on its resource share, which is updated before each event to take into account changes in the cell's density due to mortality and movement of other individuals within the time step. Only individuals with a resource share beyond 0.5 are capable of reproducing. The newly produced offspring join the population only at the end of the time step, and become adults upon commencement of the following time-step. The model is illustrated in Figures 1 and 2.

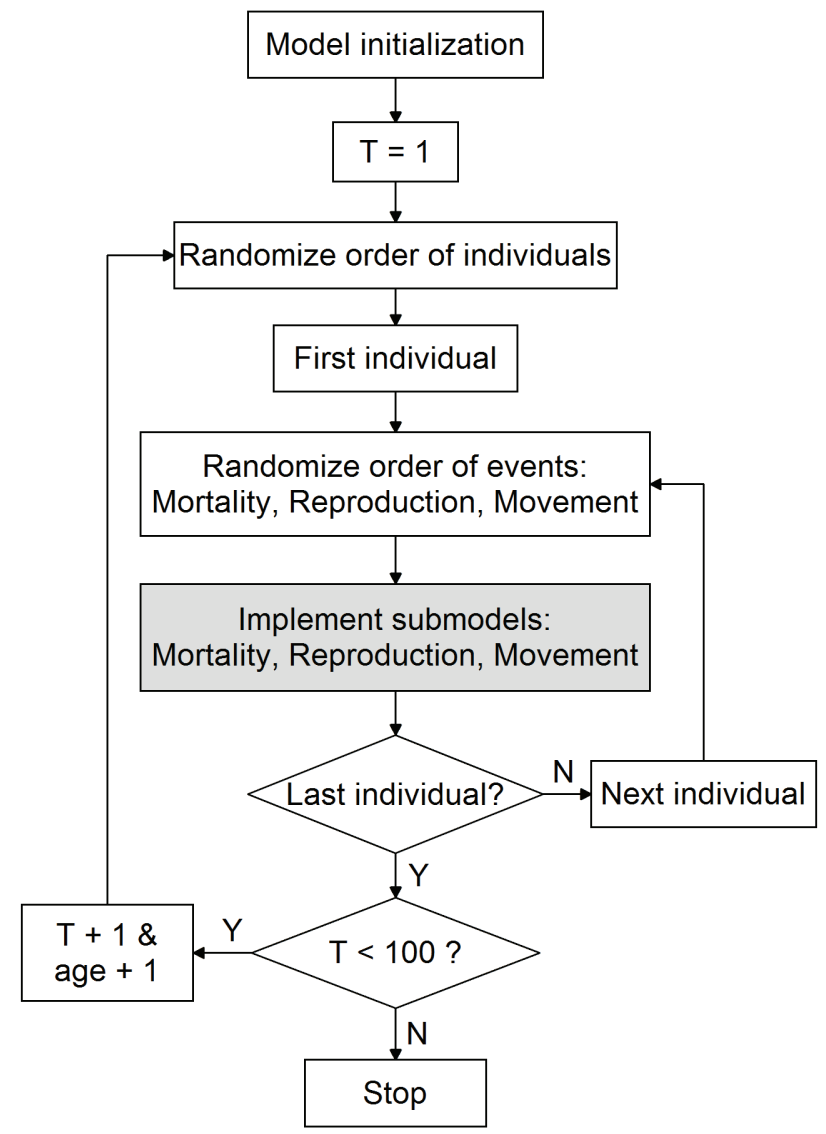

Figure 1 Flow diagram of the main routine used in the simulation model. 


\subsubsection{Design concepts}

Emergence - Population dynamics emerge from individual and species traits. Individuals' life cycles and movements are imposed by stochastic rules.

Interaction - Individuals within the same cell affect each other through the "scramble competition" (Hassell, 1975) that the resource share $(F)$ per individual declines with an increasing number of individuals within a cell. Therefore, an individual's resource share is determined by habitat quality of the inhabited cell and crowding in that cell, i.e., $F=Q / n$, where $Q$ is the habitat quality of the cell, and $n$ is the number of individuals in the cell. Direct interaction between individuals of different cells is not considered in the model.

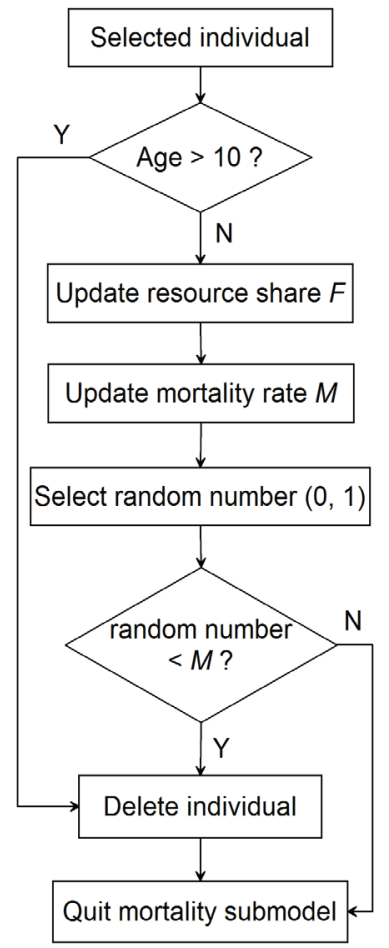

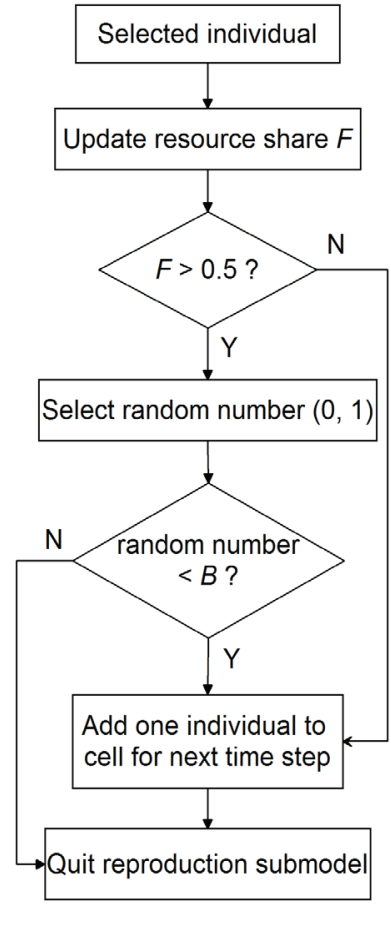

C

Selected individual

Update resource share $F$

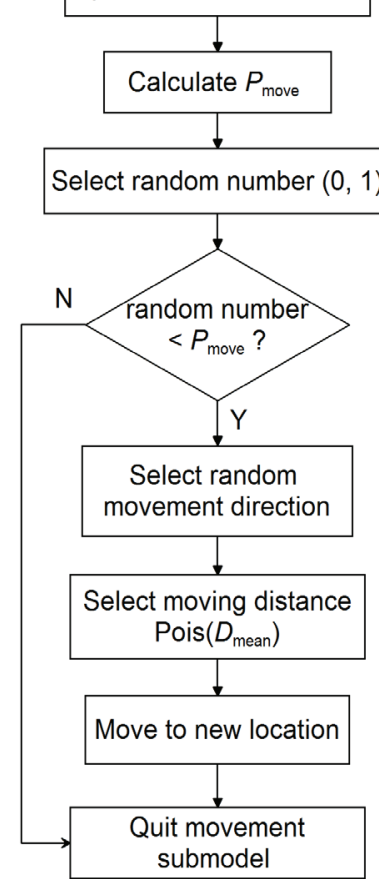

Figure 2 Flow diagrams of the submodels in the simulation model that determine mortality (a), reproduction (b), and (c) movement for a single individual within a time step. 
Stochasticity - All modelled processes are implemented stochastically via a random order of individuals in the main routine, a random order of events, and moving in a random direction. Movement, reproduction, and mortality events are implemented based on probabilities.

Observation - Population dynamics are quantified by recording the following variables throughout the last 30 time steps of each run: 1) population size; 2) mean resource share of individuals; 3 ) mean mortality rate of individuals; 4) proportion of individuals in high-quality cells $(Q \geq 0.5)$; and 5) proportion of individuals experiencing competition. By using the proportion of individuals under different conditions (e.g., residing in high-quality cells and/or experiencing competition), the absolute number of individuals are transformed to a range $0-1$, allowing direct comparison between population dynamics of different species, and thereby correcting for differences in their population sizes. For model analysis, each response variable is averaged over the last 30 time steps of each run.

\subsubsection{Initialisation}

At the beginning of each run, a two-dimensional landscape with $30 \times 30$ grid cells is generated using an unconditional Gaussian variogram model (sill = 0.025 , nugget $=0$, and range $=S$ ), where the range parameter defines the scale of spatial autocorrelation in habitat quality. The mean habitat quality of the landscape is fixed at 0.5. After generating a landscape, one hundred individuals are distributed at random through the landscape. The resource share of each individual is calculated based on the habitat quality and crowdedness of the occupied cell. Individuals' ages are independently drawn from the uniform distribution in the interval $(0$, lifespan), because preliminary tests based on different distributions (e.g., Poisson distribution) revealed that different initial age structure of individuals had no effect on population dynamics after the first 30 time-steps (see Appendix 1).

\subsubsection{Input}

There are no external environmental variables that drive the dynamics of the model.

\subsubsection{Submodels}

Mortality submodel - Probabilities of mortality are individual-specific and depend on their resource share per time step. An individual's mortality rate is calculated as: $M=M_{0} \times\left(1+\exp \left(-\left(F \times C_{\text {envir }}\right)^{2}\right)\right)$, where $M_{0}$ is the basic mortality rate, $F$ is the resource share of the individual, and $C_{\text {envir }}$ is the level of environmental tolerance. In biological terms, $C_{\text {envir }}$ regulates the sensitivity 
of individual mortality rate to environmental variability (Figure 3a). Individuals that reach the maximal lifespan are "killed" from the population.

Reproduction submodel - Only adult individuals with a high resource share (i.e., $F \geq 0.5$ ) can reproduce, giving birth to one offspring with a constant probability of $B$. More complex relations, e.g., making reproduction probability and number of offspring function of habitat quality, would by virtue of Jensen's inequality prohibit comparison between landscapes (Ruel \& Ayres, 1999; Stoddard, 2010). The newly produced offspring are attached to their mothers (i.e., in the same cells), and join the population at the end of the current time step. They become adults able to move and reproduce at the following time step.

(a)

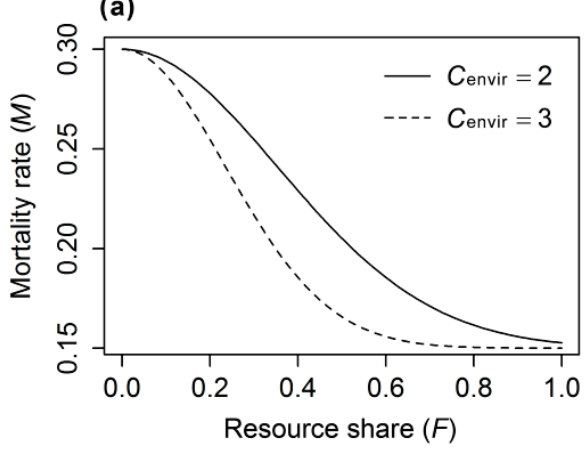

(b)

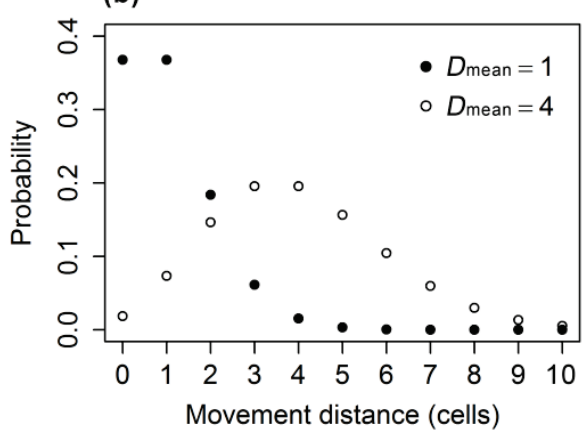

Figure 3 Movement distance distributions (a) and mortality rate functions (b) used to define hypothetical species in the model. $C_{\text {envir }}=2$ and 3 represent low-tolerant and high-tolerant species, respectively; and $D_{\text {mean }}=1$ and 4 represent low-mobility and high-mobility species, respectively.

Movement submodel - Individual movement is a conditional response to local habitat quality and crowdedness, i.e., the condition-dependent dispersal (Bowler \& Benton, 2005; Armsworth, 2009), and the probability of movement per time step is calculated as: $P_{\text {move }}=1-Q / n$, where $Q$ is the habitat quality of the cell, and $n$ is the number of individuals in the cell. Ecologically, it means that individuals residing in cells of low habitat quality and/or high density tend to depart the cell, while those residing in cells of high quality and/or low density are more likely to stay in the current cell. The movement direction per time step is randomly determined, and the movement distance per time step is drawn from a Poisson distribution with mean $\lambda=D_{\text {mean }}$ (Figure $3 b$ ). The assumption of random walk seems to be unrealistic for species that may actively search the landscape, but is conservative in the sense that effects of spatial heterogeneity are easy to detect (Fahrig, 1998). From the moving individuals' perspective, the landscape is "wrapped", meaning that an individual that crosses the edge of the landscape continues in the same direction on the opposite edge (Haefner et al., 1991). 

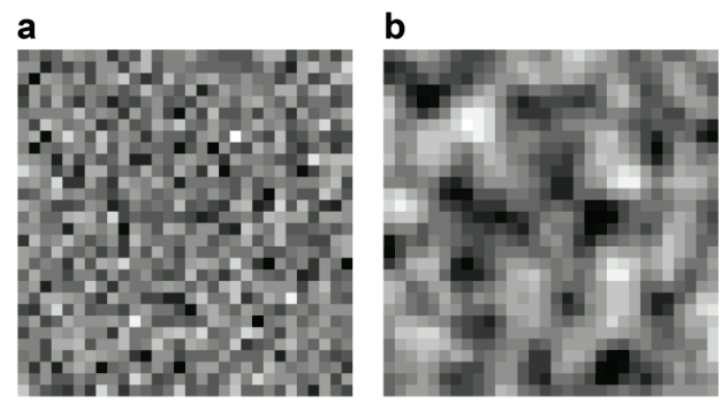

C

d
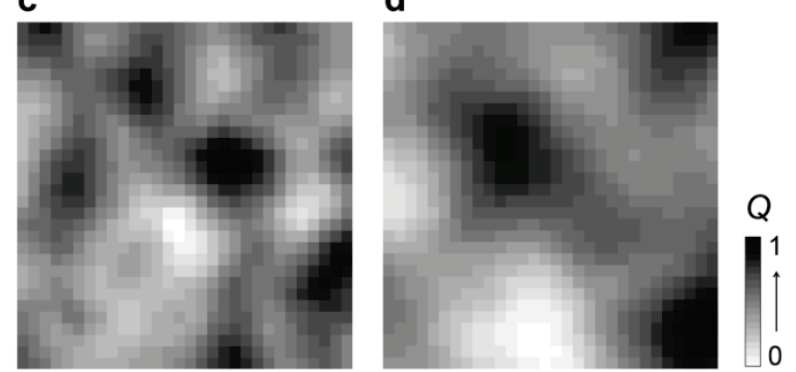

Figure 4 Examples of artificial landscapes differing in the scale of spatial autocorrelation in habitat quality $S$. (a) nearly random, $S=0.01$; (b) smallscale, $S=2$; (c) intermediate-scale, $S=4$; and (d) large-scale, $S=8$. The mean habitat quality of the landscapes is fixed at 0.5 .

\subsubsection{Simulation experiments}

To investigate how spatial autocorrelation of habitat quality interacts with species traits to affect population dynamics, we defined four landscape scenarios that reflect different levels of spatial autocorrelation in habitat quality: apparently uncorrelated $(S=0.01)$, small-scale $(S=2)$, intermediate-scale $(S=4)$, and large-scale $(S=8)$ spatial autocorrelation in habitat quality (Figure 4). For each landscape scenario, we compared the population dynamics of four virtual species differing in the level of environmental tolerance $\left(C_{\text {envir }}=2\right.$ or 3 ; Figure $3 a$ ) and mean movement distance $\left(D_{\text {mean }}=1\right.$ or 4 cells; Figure $\left.3 \mathrm{~b}\right)$. The combination of landscape scenarios and species classes resulted in a total of 16 treatments $(4 \times 4=$ 16 ) in the experiments. For each treatment, we conducted 50 replicates, each lasting for 100 time steps. This is based on preliminary runs using 200 time-steps, in which we found that any population reached a state of dynamic equilibrium within the first 50 time-steps.

The flexibility of agent-based models means that they typically have a large number of parameters. Ideally, parameter values would be estimated from empirical data. Unfortunately, such data are scarce, and the number of parameters is so large that it is not possible to investigate every combination 
of parameter values. Therefore, following a previous sensitivity analysis (data not shown), parameter values were chosen arbitrarily to yield an overall rate of population increase of $\sim 1$ over the first 50 time steps for the species with $C_{\text {envir }}=2$ and $D_{\text {mean }}=1$ in landscapes with apparently uncorrelated habitat quality (i.e., landscape scenario $S=0.01$ ). Real-world species would experience life-history trade-offs in demographic and movement rates which influence population growth (Pearson \& Fraterrigo, 2011). To permit a more direct comparison of the combined impacts of changing life-history traits and habitat spatial structure, we assumed that all virtual species had the same basic mortality rate $\left(M_{0}\right)$ and birth probability $(B)$. Since we were interested in combining landscape spatial autocorrelation with species traits, these assumptions were considered acceptable. The detailed parameter values are given in Table 1.

Table 1 Variables, parameters, and initial conditions used in the model.

\begin{tabular}{lll}
\hline Parameter & \multicolumn{2}{l}{ Symbol } \\
\hline Landscape size & - & $30 \times 30$ cells \\
Habitat quality of cells & $Q$ & $0-1$ \\
Mean habitat quality of landscape & - & 0.5 \\
Scale of spatial autocorrelation in habitat quality & $S$ & $0.01,2,4,8$ \\
Individual's resource share & $F$ & $0-1$ \\
Birth probability & $B$ & 0.7 \\
Basic mortality rate & $M_{0}$ & 0.15 \\
Level of environmental tolerance & $C_{\text {envir }}$ & 2,3 \\
Mean moving distance & $D_{\text {mean }}$ & 1,4 cells \\
Litter size & - & 1 \\
Max. lifespan & - & 10 time steps \\
Initial population size & - & 100 \\
Length of simulation period & - & 100 time steps \\
\hline
\end{tabular}

\subsubsection{Statistical analysis}

We first performed a Shapiro-Wilks normality test to examine data normality and found that data were normally distributed. To assess the main effects and interactions among the three experimental factors (i.e., scale of spatial autocorrelation in habitat quality $S$, species' environmental tolerance $C_{\text {envir }}$, and mean moving distance $D_{\text {mean }}$ ), we estimated the magnitude of effects of the variance sources using a three-way analysis of variance (ANOVA), as suggested by White (White et al., 2014). We performed three-way ANOVA for each of the five response variables (cf. Observation section). The three 
experimental factors were treated as categorical variables in accordance with the experimental design. The effect sizes of the factors and interactions were measured by percentage of variance explained (Cohen, 1988) and generalized eta-squared (Olejnik \& Algina, 2003; Bakeman, 2005). For each treatment, we also investigated Spearman correlations of the mean resource share of individuals with the proportion of individuals in high-quality cells and with the proportion of individuals experiencing competition. By doing so, we aimed at increasing our understanding of the mechanisms behind observed population dynamics in different landscape scenarios. All Data analyses were conducted in the R environment with the 'ez' package (Lawrence, 2011).

\subsection{Results}

All hypothetical species exhibited a constant increase in population size as the scale of spatial autocorrelation in habitat quality increased (Figure 5a). However, the pattern of the increase in population size varied greatly between the species with different movement capacities. Low-mobility species $\left(D_{\text {mean }}=1\right)$ displayed a logarithmic-like increase in response to increased spatial autocorrelation, contrasting with the exponential-like increase of high-mobility species $\left(D_{\text {mean }}=4\right)$. Under the same level of species' environmental tolerance, low-mobility species showed greater population size than high-mobility species in all landscape scenarios except for the scenario of $S=0.01$ (Figure $5 a$ ).

The analysis of variance revealed that both species' environmental tolerance and the scale of spatial autocorrelation in habitat quality had strong effects on population size, accounting for $\sim 47 \%$ and $\sim 33 \%$, respectively, of the variation in population size (Table 2 , also see Appendix 2). Their interaction also had a significant effect $\left(S \times D_{\text {mean }}: F_{7,792}=487.96, p<0.001\right)$, but accounted only for $\sim 1 \%$ of the variation in population size (Table 2 ). Species' movement capacity and its interaction with scale of spatial autocorrelation in habitat quality also had appreciable effects and explained $~$ $10 \%$ of the variation in population size $\left(D_{\text {mean }}: F_{1,798}=23.04, p<0.001\right.$; $\mathrm{S} \times \mathrm{D}_{\text {mean }}: F_{7,792}=85.29, p<0.001 ;$ Table 2$)$.

All virtual species also showed an exponential increase in mean resource share as the scale of spatial autocorrelation in habitat quality increased (Figure 5b), and low-tolerant species had appreciably higher mean resource shares than high-tolerant populations (Mann-Whitney test, $p<0.01$ ). Species' environmental tolerance had the greatest effect on individual resource share, explaining $\sim 62 \%$ of the variation in mean resource share (Table 2, also see Table S1). Scale of spatial autocorrelation in habitat quality accounted for $\sim 15 \%$ of the variation in mean resource share of individuals $(S$; Table 2). In addition, the effect of movement capacity on mean resource 
share was appreciably greater when habitat quality was spatially autocorrelated at a broad scale $\left(S \times D_{\text {mean }}: F_{7,792}=21.18, p<0.001\right)$.
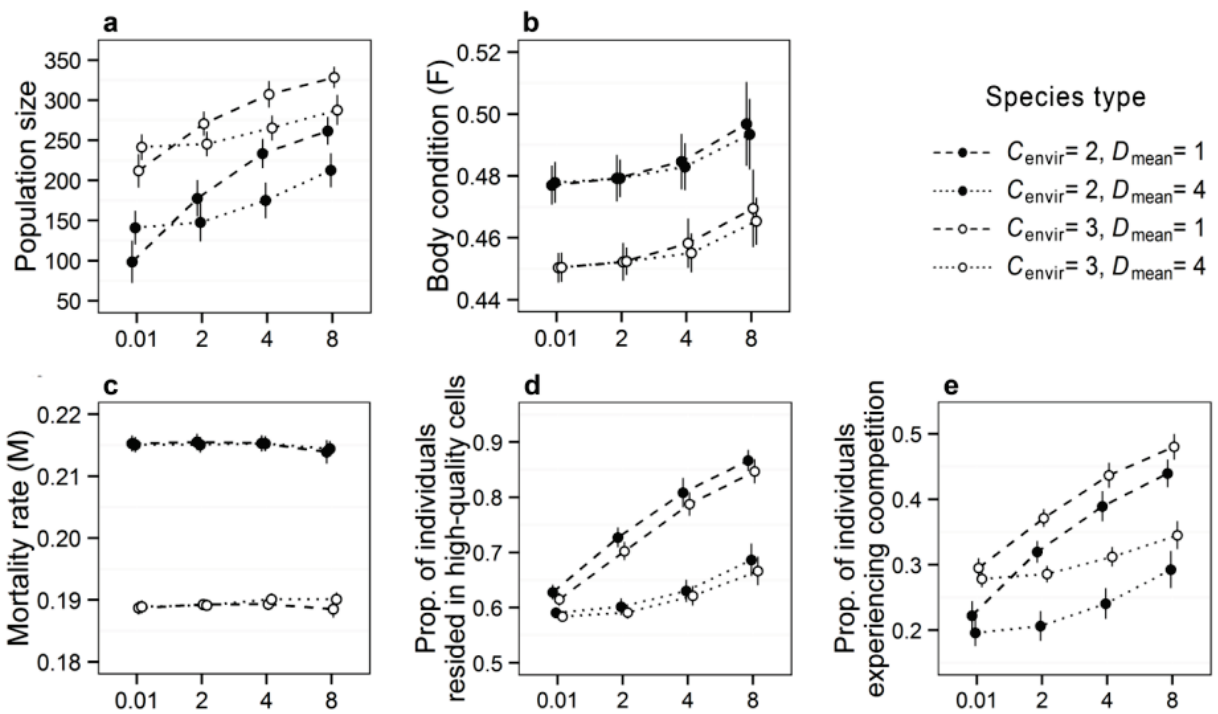

Scale of spatial autocorrelation in habitat quality $(S)$

Figure 5 Population responses to the scale of spatial autocorrelation in habitat quality $S$ ( $x$-axes, varied from random to broad-scale) for hypothetical species that differ in aspects of environmental tolerance $C_{\text {envir }}$ and mean moving distance $D_{\text {mean }}$ (cf. Simulation Experiments section): a Population size, b mean resource share of individuals, $c$ mean mortality rate of individuals, $d$ Proportion of individuals in high-quality cells $(Q \geq 0.5)$, and e Proportion of individuals experiencing competition. The figure shows mean \pm 1 SD for $\mathbf{5 0}$ replicates for each variable. 


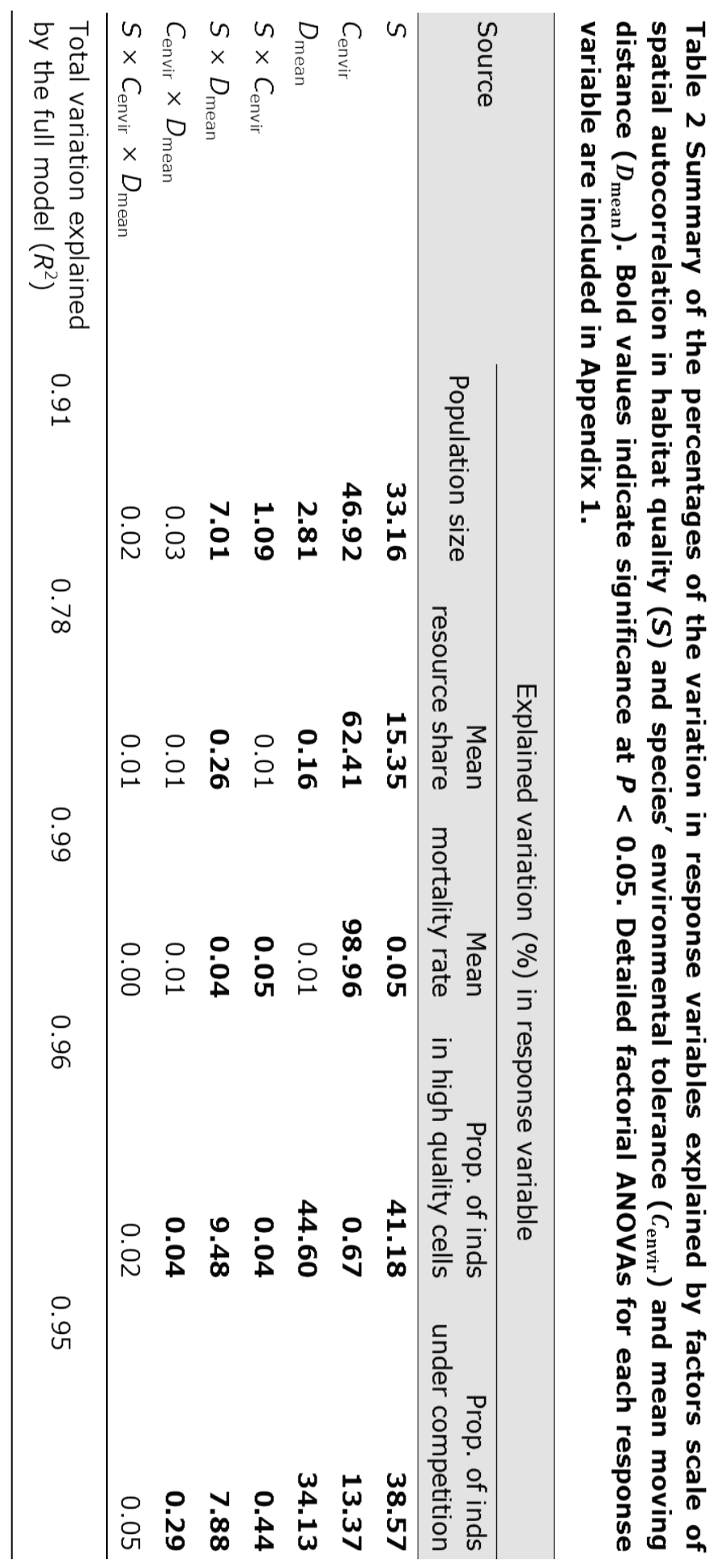




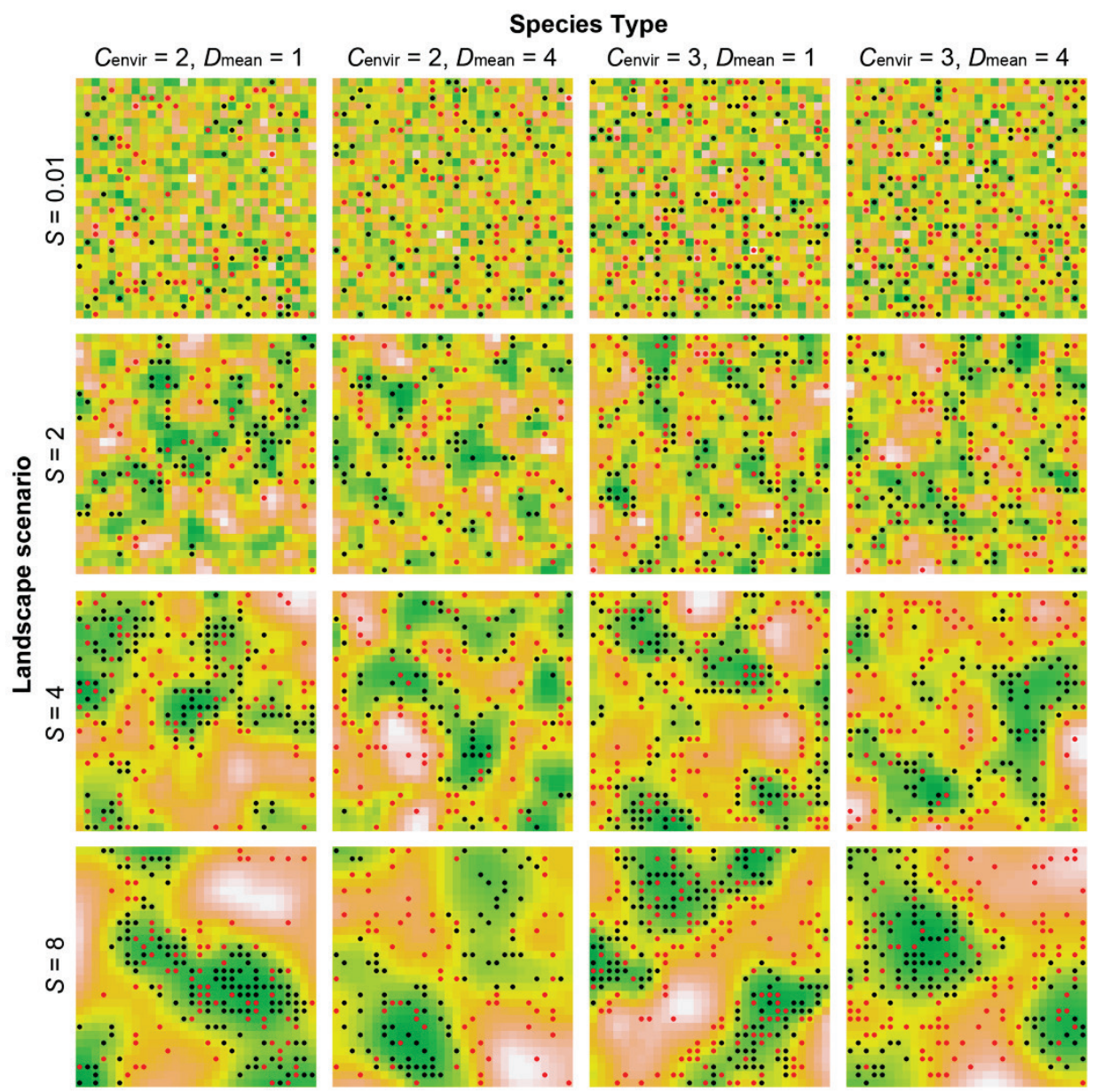

Figure 6 Sample patterns of spatial distribution of individuals under different landscape scenarios. Virtual species are parameterized by environmental tolerance $C_{\text {envir }}$ and mean moving distance $D_{\text {mean }}$. Black dots represent individuals residing in cells of $Q \geq 0.5$ and free of competition, while red dots are individuals residing in cells of $Q<0.5$ or experiencing competition. Greener colour indicates higher habitat quality.

For the mean mortality rate of individuals, species' environmental tolerance had a dominant effect and accounted for $~ 99 \%$ of the variation (Table 2, also see Appendix 2). In contrast, the scale of spatial autocorrelation in habitat quality and species' movement capacity had little effect on the mean mortality rate of individuals, due to the dominant effect of species' environmental tolerance (Figure5c and Table 2).

The proportion of individuals residing in high-quality cells and the proportion of individuals experiencing competition reflect the spatial distribution of individuals in response to the spatial structure of habitat quality (Figure 6, 
also see Appendices $3 \& 4$ ). Increasing the scale of spatial autocorrelation in habitat quality increased the proportion of individuals residing in high-quality cells, especially for low-mobility species (Figure $5 d$, also see Appendix 3 ). Species' movement capacity and the scale of spatial autocorrelation in habitat quality both had strong effects, accounting for $\sim 45$ and $41 \%$, respectively, of the variation in the proportion of individuals in high-quality cells (Table 2, also see Appendix 2). Their interaction also had a significant effect and explained $\sim 9 \%$ of the variation $\left(S \times D_{\text {mean }}: F_{7,792}=2273.5, p<0.001\right)$.

Likewise, the proportion of individuals experiencing competition increased as the scale of spatial autocorrelation in habitat quality increased, and lowmobility species had higher proportions when habitat quality was spatially autocorrelated (Figure5e, also see Appendix 4). The scale of spatial autocorrelation in habitat quality had the greatest effect on the proportion of individuals experiencing competition, followed by species' movement capacity and environmental tolerance (Table 2, also see Appendix 2). The interaction between the scale of spatial autocorrelation in habitat quality and species' movement capacity also had a significant effect, accounting for $\sim 8 \%$ of the variation in the proportion of individuals experiencing competition $\left(S \times D_{\text {mean }}\right.$ : $\left.F_{7,792}=469.54, p<0.001\right)$.

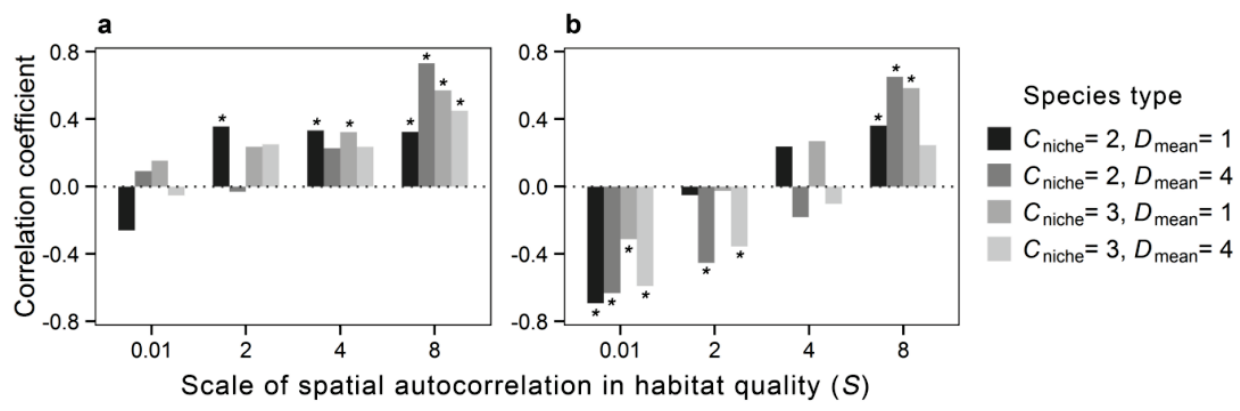

Figure 7 Coefficients of Spearman correlations of the mean resource share of individuals with the proportion of individuals in high-quality cells (a) and with the proportion of individuals experiencing competition (b) for four hypothetical species under different landscape scenarios. Bars marked with an asterisk $(*)$ indicate the coefficients are statistically significant at $P<$ 0.05 .

In general, the mean resource share of individuals was positively associated with the proportion of individuals in high-quality cells when habitat quality was spatially autocorrelated at a broad scale (Figure 7a). In contrast, the associations between mean resource share of individuals and the proportion of individuals experiencing competition were differential, depending on the spatial structure of habitat quality. When the spatial structure of habitat quality shifted from spatially random to autocorrelated at a broad scale, the 
correlation changed from negative to positive (Figure 7b), indicating that local movement in combination with spatial autocorrelation can actually increase population size and resource share despite of increased density effects.

\subsection{Discussion}

In this study, we explored how habitat spatial autocorrelation interacts with species' life-history attributes to influence population dynamics within spatially structured landscapes. We found that increasing the scale of spatial autocorrelation in habitat quality can greatly increase the population size as well as mean resource share of virtual species and in line with common expectation, high-tolerant species had an appreciably higher population size than low-tolerant species.. These results are generally consistent with previous findings that more specialized species are more affected by habitat spatial heterogeneity (Gonzalez \& Holt, 2002; Bolker, 2003; Zając et al., 2008; Ye et al., 2013b). These observed patterns may be partly explained by edge effects, which is one of the factors influencing population dynamics (Ries et al., 2004). As the scale of spatial autocorrelation increases, habitat quality becomes less rugged across space, forming fewer but larger suitable areas in the landscape, and consequently reduces changes in abundance or fitness of low-tolerant species with respect to low quality of "edge" areas. In contrast, the high-tolerant species, due to higher tolerances to variation in habitat quality, may be insensitive to such edge effects (Pearson \& Fraterrigo, 2011). Moreover, our results are comparable to the population dynamics in the "source-sink" context. Spatial autocorrelation in habitat quality can result in neighbourhoods of high-quality and low-quality areas, thereby creating source-sink dynamics in relation to habitat heterogeneity (Dhondt et al., 1992; Rodenhouse et al., 1997). If a landscape is spatially autocorrelated (particularly at large extents), individuals dispersing away from "source" habitats are more likely to encounter higher quality habitats rather than disperse into a demographic sink.

Species' movement capacity can interact with habitat heterogeneity to influence population dynamics and distribution of individuals (Snyder \& Chesson, 2003; Pinto \& MacDougall, 2010). Our simulation demonstrated that low-mobility species had greater population sizes than high-mobility species in the presence of spatial autocorrelation in habitat quality. The findings also suggest that species' movement capacity plays an important role in shaping the increase in population size in response to an increased spatial autocorrelation in habitat quality, where low-mobility species had a logarithmic-like increase in response to increased scale of spatial autocorrelation, contrasting with the exponential-like increase for highmobility species. This may be because, under the assumption of random 
walk, individuals with short-distance movement are likely to stay in suitable habitats, while long-distance movement will increase the risk of landing in an unsuitable habitat when habitat quality is autocorrelated at fine scales (With \& King, 1999; Bolker, 2003; Hiebeler, 2004). Such contrasting patterns of population increase in relation to movement capacity indicate that the effect of distance-based movement capacity may function in highly scale-dependent ways, and its relative strength is determined by interactions among movement distance, the scale of spatial autocorrelation, and the effects of species competition (Snyder \& Chesson, 2003; Gilbert \& Lechowicz, 2004; Pinto \& MacDougall, 2010). For instance, the logarithmic-like increase in population size of low-mobility species in response to increased spatial autocorrelation indicates that the benefit from short-distance movement was buffered by increasing effects of population density, whereas the exponentiallike increase in population size for high-mobility species may suggest that long-distance movement will reduce competitive interactions between individuals, thereby increasing the net population. Skelsey and Garrett (2013) predicted that the magnitude of dispersal (number of individuals) should be maximized at intermediate scales of heterogeneity, i.e., when the scale of spatial heterogeneity is neither too fine nor too coarse relative to the movement capacity of a species. This potentially provides an additional explanation for the observed patterns in population dynamics in response to spatial autocorrelation in habitat quality.

The population dynamics of species inhabiting complex landscapes generally involve two major components: the dispersal of individuals and habitatspecific mortality rates (Pulliam \& Danielson, 1991). The interplay between spatial autocorrelation in habitat quality and species life-history traits can lead to spatial aggregation of individuals, creating variation in demographic performance among individuals (Dhondt et al., 1992; Rodenhouse et al., 1997). This is evident by our model, where the proportions of individuals in high-quality cells and the proportion of individuals experiencing competition increased differentially in response to increased spatial autocorrelation between species with different movement capacity (see Figure S1 and Figure S2). These results indicate that localized movement in combination with habitat spatial structure may increase the population size despite of increased density effects in the presence of spatial autocorrelation in habitat quality. Furthermore, we found that the mean resource share was positively correlated with the proportion of individuals experiencing competition when habitat quality was spatially autocorrelated. This may sound contradictory, because an individual's resource share would be, by definition, reduced by increased local crowding. However, increasing spatial autocorrelation in habitat quality could also improve the quality of individuals by increasing the probability of finding high-quality habitat within the range of movement, which may buffer the density-dependent effects on the population (Schiegg, 
2003). Such complex relationships indicate how the interplay of movement capacity and environmental autocorrelation can jointly influence the outcome of competitive interactions (Nevoux et al., 2010).

The results of this simulation are contingent upon numerous simplifying model assumptions, and it remains to be seen whether the same or similar effects also occur for species living in real landscapes. For example, the model used a simplified landscape with the edge being "wrapped" so that an individual crossing the edge of the landscape continues in the same direction on the opposite edge (Haefner et al., 1991). However, moving individuals would experience sharp discontinuities as they cross the edges if the simulated landscape boundaries are not periodic. To avoid this, one can generate landscapes with either periodic boundary conditions or with a larger spatial extent, depending on the study of interest (Chipperfield et al., 2011). Furthermore, we assumed individuals move in a random manner. The random movement assumption seems to be unrealistic for species that may actively search the landscape within their home range (e.g. birds and mammals). However, if individuals can perceive and orient new habitat location from some distance, it may result in a reduced effect of habitat spatial structure on population dynamics. Therefore, the random movement assumption is conservative in the sense that effects of landscape heterogeneity are easy-to-detect (Fahrig, 1998). Moreover, male individuals were not modelled based on the perspective that females form the reproductive unit and thereby determine the population dynamics (Pulliam et al., 1992; Wiegand et al., 1999). We also excluded life-history trade-offs in demographic and movement rates by assuming that only mortality rate is associated with local habitat quality and density. However, more complex relations, e.g., making both mortality and reproduction functions of habitat quality, would increase the unpredictability in model outputs and prohibit comparison between landscapes due to the Jensen's inequality (Ruel \& Ayres, 1999; Stoddard, 2010).

Although the model incorporates numerous simplifying assumptions, the general prediction that low-mobility species will have greater sensitivity to changing scale of spatial autocorrelation in habitat quality strongly suggests that mechanisms shaping population dynamics are complex and depend on both habitat spatial heterogeneity and species' life history traits considered. We have yet to confirm them in the field, but if the spatial autocorrelation favouring species exists, we may expect a nonlinear relationship between local population dynamics and habitat heterogeneity in the sense that species movement limitation represents a threshold (Schooley \& Branch, 2007). Nevertheless, understanding which mechanisms in spatially structured landscapes regulate a population is important for explaining species abundances and designing management plans for species conservation. 


\section{Acknowledgments}

The authors would like to thank Lalit Kumar and two anonymous reviewers for suggestions for improvements on this article. This work was financially supported by the Erasmus Mundus External Co-operation Window Programme (EMECW) of the European Union and co-funded by the ITC Research Fund. 


\section{Appendix 1}

Testing for the effects of initial age-structures on model output.

We simulated the dynamics of populations under different initial agestructures: uniform distribution, poisson distribution with $\lambda=2$, and poisson distribution with $\lambda=5$. We found no significant differences among population sizes under different initial age-structures (Kruskal-Wallis test, $p<0.05$; Figure S1). The age structure of populations at the $50^{\text {th }}$ time step of the simulations under different initial age-structures also showed little difference (Figure S2).

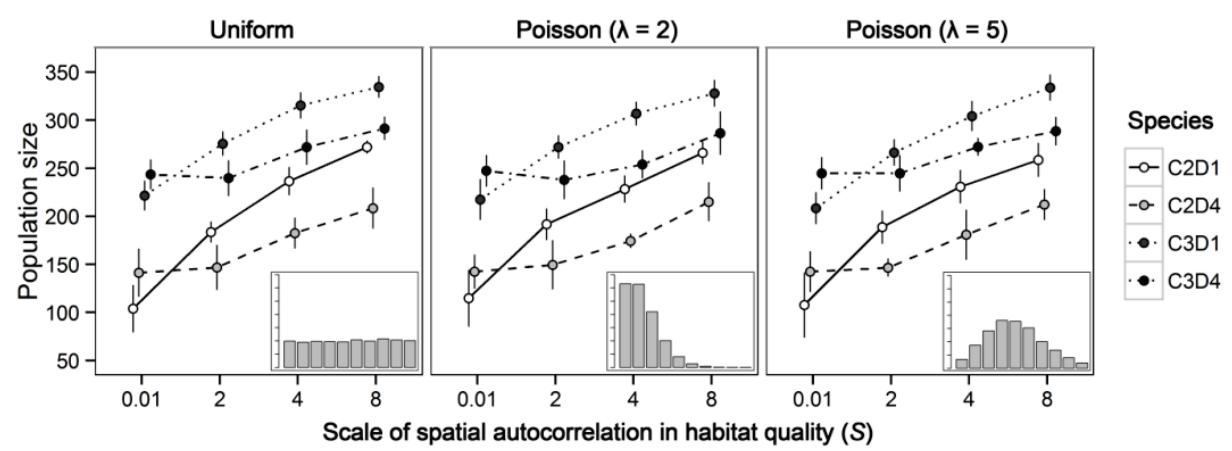

Figure S1. simulated population sizes under different initial age structure distributions: uniform distribution, poisson distribution with $\lambda=2$, and poisson distribution with $\lambda=5$. The inserts are the distributions of initial age distributions of populations. 

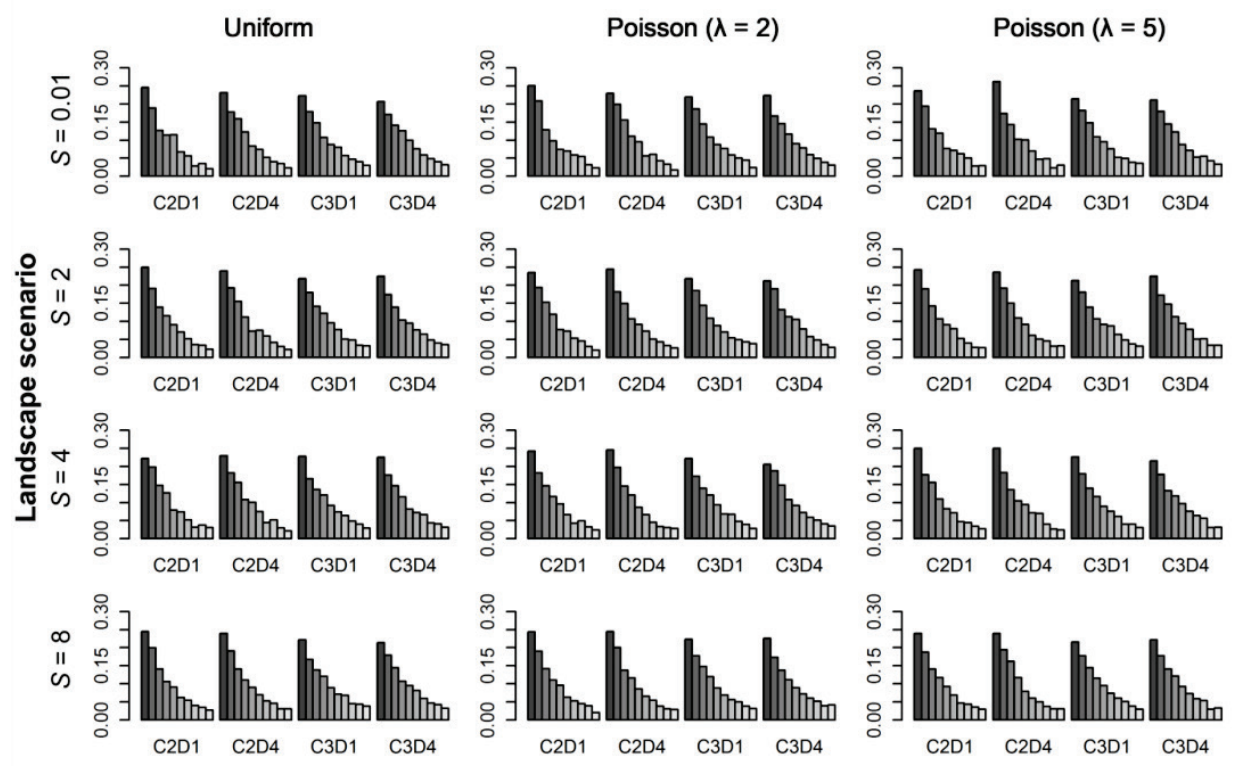

Figure S2. Histograms of age of individuals at the $50^{\text {th }}$ time step of the simulation under different initial age-structure distributions: uniform distribution, poisson distribution with $\lambda=2$, and poisson distribution with $\lambda=$ 5. 


\section{Appendix 2}

Table A1 Factorial ANOVAs on each of the five response variables under different scale of spatial autocorrelation in habitat quality (S) and species' environmental tolerance ( $C_{\text {envir }}$ ) and movement capacity $\left(D_{\text {mean }}\right)$. The $F$-ratio of the variance between groups to the variance within groups. Percentage of $S S_{\text {total }}$ is defined as the sum of squares of a factor relative to total sum of squares, i.e., $S_{\text {effect }} / S S_{\text {total }} \cdot \eta_{\mathrm{G}}^{2}$ is the generalized eta squared.

\begin{tabular}{|c|c|c|c|c|c|}
\hline Source of variation & $d f$ & F-ratio & $P$-value & $\%$ of $S S_{\text {total }}$ & $\eta_{\mathrm{G}}^{2}$ \\
\hline \multicolumn{6}{|l|}{ Population size } \\
\hline$S$ & 3 & 984.24 & $<0.001$ & 33.16 & 0.79 \\
\hline$C_{\text {envir }}$ & 1 & 4178.39 & $<0.001$ & 46.92 & 0.84 \\
\hline$D_{\text {mean }}$ & 1 & 249.83 & $<0.001$ & 2.81 & 0.24 \\
\hline$S \times C_{\text {envir }}$ & 3 & 32.42 & $<0.001$ & 1.09 & 0.11 \\
\hline$S \times D_{\text {mean }}$ & 3 & 208.21 & $<0.001$ & 7.01 & 0.44 \\
\hline$C_{\text {envir }} \times D_{\text {mean }}$ & 1 & 2.32 & 0.128 & 0.03 & 0.00 \\
\hline$S \times C_{\text {envir }} \times D_{\text {mean }}$ & 3 & 1.08 & 0.432 & 0.02 & 0.00 \\
\hline Residuals & 784 & & & 8.95 & \\
\hline \multicolumn{6}{|c|}{ Mean resource share of individuals } \\
\hline$S$ & 3 & 184.02 & $<0.001$ & 15.35 & 0.41 \\
\hline$C_{\text {envir }}$ & 1 & 2244.84 & $<0.001$ & 62.41 & 0.74 \\
\hline$D_{\text {mean }}$ & 1 & 5.88 & 0.016 & 0.16 & 0.01 \\
\hline$S \times C_{\text {envir }}$ & 3 & 0.10 & 0.963 & 0.01 & 0.00 \\
\hline$S \times D_{\text {mean }}$ & 3 & 3.11 & 0.026 & 0.26 & 0.01 \\
\hline$C_{\text {envir }} \times D_{\text {mean }}$ & 1 & 0.37 & 0.541 & 0.01 & 0.00 \\
\hline$S \times C_{\text {envir }} \times D_{\text {mean }}$ & 3 & 0.10 & 0.961 & 0.01 & 0.00 \\
\hline Residuals & 784 & & & 21.80 & \\
\hline \multicolumn{6}{|c|}{ Mean mortality rate of individuals } \\
\hline$S$ & 3 & 13.83 & $<0.001$ & 0.05 & 0.05 \\
\hline$C_{\text {envir }}$ & 1 & 88415.67 & $<0.001$ & 98.96 & 0.99 \\
\hline$D_{\text {mean }}$ & 1 & 10.20 & 0.002 & 0.01 & 0.01 \\
\hline$S \times C_{\text {envir }}$ & 3 & 14.52 & $<0.001$ & 0.05 & 0.05 \\
\hline$S \times D_{\text {mean }}$ & 3 & 11.41 & $<0.001$ & 0.04 & 0.04 \\
\hline$C_{\text {envir }} \times D_{\text {mean }}$ & 1 & 13.28 & $<0.001$ & 0.01 & 0.02 \\
\hline$S \times C_{\text {envir }} \times D_{\text {mean }}$ & 3 & 1.12 & 0.341 & 0.00 & 0.00 \\
\hline Residuals & 784 & & & 0.88 & \\
\hline
\end{tabular}

(Continued on next page) 
Table A1 (continued)

\begin{tabular}{lrrrrr}
\hline Source of variation & $d f$ & F-ratio & $P$-value & $\%$ of $S S_{\text {total }}$ & $\eta_{\mathrm{G}}^{2}$ \\
\hline Prop. of individuals resided in high-quality cells & & & & \\
$S$ & 3 & 2715.10 & $<0.001$ & 41.18 & 0.91 \\
$C_{\text {envir }}$ & 1 & 132.65 & $<0.001$ & 0.67 & 0.14 \\
$D_{\text {mean }}$ & 1 & 8820.83 & $<0.001$ & 44.60 & 0.92 \\
$S \times C_{\text {envir }}$ & 3 & 2.68 & 0.046 & 0.04 & 0.01 \\
$S \times D_{\text {mean }}$ & 3 & 625.01 & $<0.001$ & 9.48 & 0.71 \\
$C_{\text {envir }} \times D_{\text {mean }}$ & 1 & 8.65 & 0.003 & 0.04 & 0.01 \\
$S \times C_{\text {envir }} \times D_{\text {mean }}$ & 3 & 1.45 & 0.228 & 0.02 & 0.01 \\
Residuals & 784 & & & 3.96 & \\
\hline Prop. of individuals experiencing competition & & & & \\
$S$ & 3 & 1914.23 & $<0.001$ & 38.57 & 0.88 \\
$C_{\text {envir }}$ & 1 & 1991.38 & $<0.001$ & 13.37 & 0.72 \\
$D_{\text {mean }}$ & 1 & 5082.07 & $<0.001$ & 34.13 & 0.87 \\
$S \times C_{\text {envir }}$ & 3 & 21.74 & $<0.001$ & 0.44 & 0.08 \\
$S \times D_{\text {mean }}$ & 3 & 391.29 & $<0.001$ & 7.88 & 0.60 \\
$C_{\text {envir }} \times D_{\text {mean }}$ & 1 & 42.90 & $<0.001$ & 0.29 & 0.05 \\
$S \times C_{\text {envir }} \times D_{\text {mean }}$ & 3 & 2.59 & 0.052 & 0.05 & 0.01 \\
Residuals & 784 & & & 5.27 & \\
\hline
\end{tabular}




\section{Appendix 3}

Sample patterns of spatial distribution of individuals in high-quality cells under different landscape scenarios. Hypothetical species are parameterized by the level of environmental tolerance $C_{\text {envir }}$ and mean moving distance $D_{\text {mean }}$. Black dots represent individuals in cells of $Q \geq 0.5$, while red dots are individuals in cells of $Q<0.5$. More green color indicates higher habitat quality. $\mathrm{N}$ is the population size.

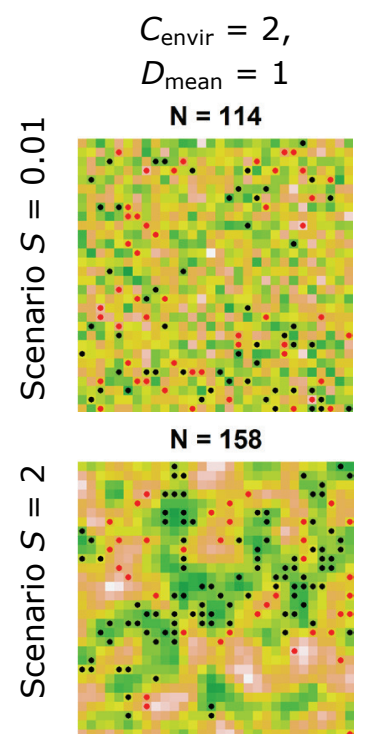

$\mathbf{N}=\mathbf{2 5 1}$

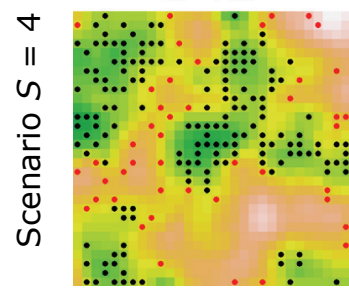

$\mathrm{N}=\mathbf{2 5 8}$

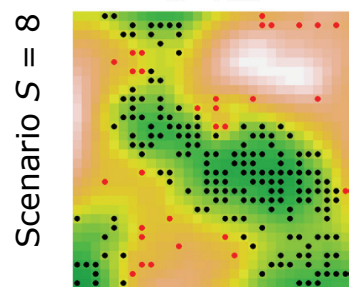

$C_{\text {envir }}=2$,

$D_{\text {mean }}=4$ $\mathrm{N}=156$

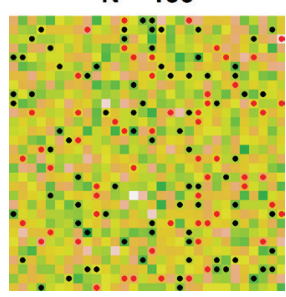

$N=129$

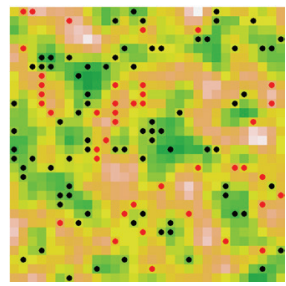

$N=184$

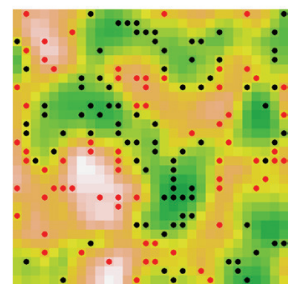

$N=166$

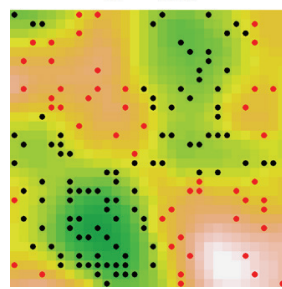

$C_{\text {envir }}=3$

$D_{\text {mean }}=1$

$\mathbf{N}=\mathbf{2 1 6}$

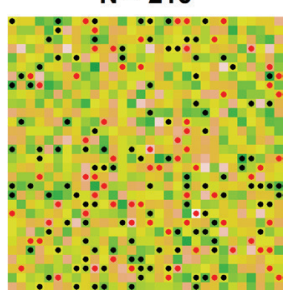

$N=250$

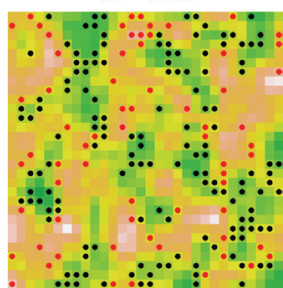

$\mathbf{N}=316$

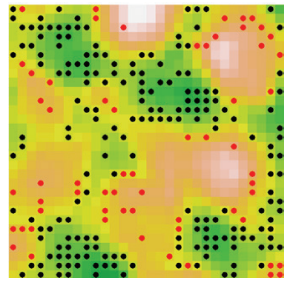

$N=346$

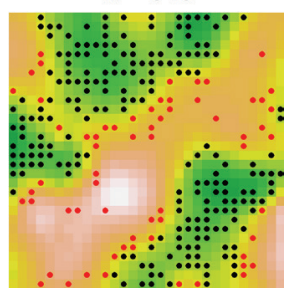

$C_{\text {envir }}=3$,

$D_{\text {mean }}=4$

$\mathbf{N}=\mathbf{2 2 9}$

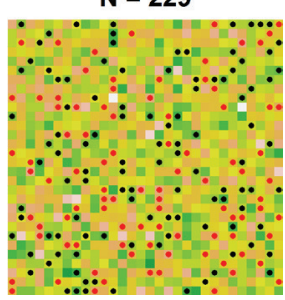

$N=241$

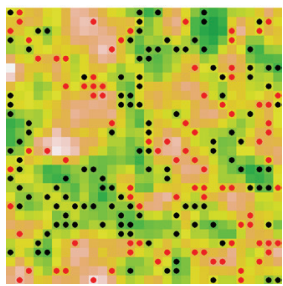

$N=238$

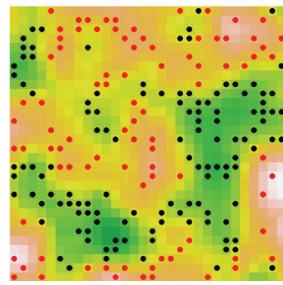

$N=284$

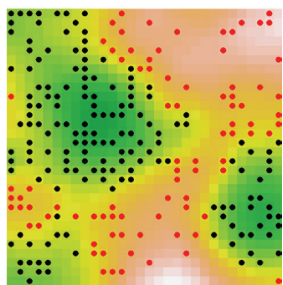




\section{Appendix 4}

Sample patterns of spatial distribution of individuals experiencing competition under different landscape scenarios. Hypothetical species are parameterized by the level of environmental tolerance $C_{\text {envir }}$ and mean moving distance $D_{\text {mean }}$. Black dots represent individuals free of competition, while red dots are individuals experiencing competition. More green color indicates higher habitat quality. $\mathrm{N}$ is the population size.

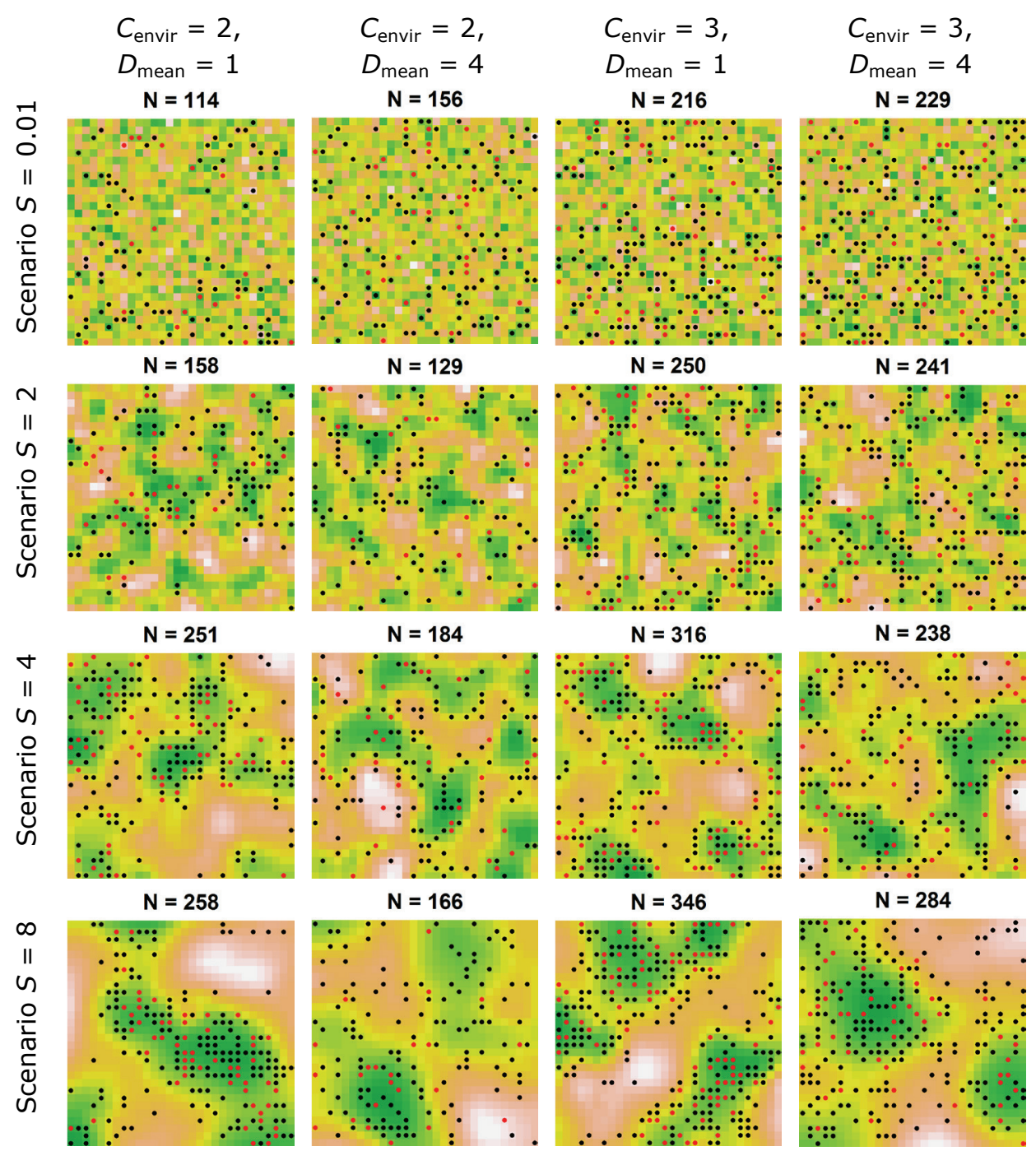




\section{A wavelet-based approach to evaluate the roles of structural and functional landscape heterogeneity in animal space use at multiple scales}

This chapter is based on: Ye, X., Wang, T., Skidmore, A., Fortin, D., BastilleRousseau, G., and Parrott, L. (2014) A wavelet-based approach to evaluate the roles of structural and functional landscape heterogeneity in animal space use at multiple scales. Ecography, 37:1-11. 


\section{Abstract}

The functional relationship between habitat utilization and landscape spatial heterogeneity is fundamental to understanding the spatial nature of animal distribution across scales. Although structural and functional properties of landscape spatial heterogeneity can have different consequences for animal species, few studies have explicitly considered both forms of heterogeneity, partly due to the lack of general methods for direct assessment of scalespecific associations between variables. We present a wavelet-based approach to evaluate the roles of structural and functional landscape spatial heterogeneity in animal space use at multiple spatial scales. As a case study, we examined scale-specific space use patterns of American black bears (Ursus americanus) in response to structural and functional spatial heterogeneity as well as spatial patterns of vegetation age-classes in a Canadian boreal forest. We found strong differences in the effects of structural and functional spatial heterogeneity and the scales at which they are associated with the patterns of habitat use by black bears. Functional heterogeneity alone affected space use at 800-m and 1600-m scales, but had significant effects when interacting with structural heterogeneity at $400-\mathrm{m}$, 800-m, and 1600-m scales. Compared with male bears, female black bears were most sensitive to patterns of forage abundance at intermediate scales, or more specifically, in young and regenerating forests that provide abundant soft mast in boreal forests. Our study highlights the importance of accounting for scale-dependent properties of (structural and functional) spatial heterogeneity in assessing the ecological effects of landscape components and the effectiveness of the wavelet transform technique in identifying such scale-specific relationships.

Keywords: spatial heterogeneity, landscape composition, landscape function, wavelet-coefficient regression, resource utilization function, Ursus americanus 


\subsection{Introduction}

Spatial heterogeneity is a fundamental characteristic of all landscapes, and its formation and interactions with ecological processes across scales are a central issue in spatial ecology (Wiens, 1989; Wu et al., 2000; Turner \& Tjørve, 2005). Depending on whether the requirements of a particular species or species group are explicitly considered or not, landscape spatial heterogeneity can be defined as either "structural heterogeneity" or "functional heterogeneity" (Kolasa \& Rollo, 1991; Fahrig et al., 2011). The former is the complexity or variability of a system property identified solely by its physical attributes (e.g., different vegetation types) and without reference to the requirements of focal species/groups, whereas the latter is based on the variability of properties that affect ecological processes relevant to the species/community of interest (e.g., foraging site and nesting habitat). From an ecological standpoint, the spatial pattern or scale of structural heterogeneity (e.g., forest types) is not necessarily equivalent to that of functional heterogeneity (e.g., food availability) and vice versa, because different landscape structural elements may function similarily for a particular species/group (Lima \& Zollner, 1996; Schick et al., 2008; Fahrig et al., 2011). Evaluating the effects of spatial heterogeneity based solely on structual properties may result in partial and potentially misleading conclusions, because the non-structural variations within or among cover types that are relevant to a species response (e.g., food abundance and nesting-site availability) cannot be fully reflected by structural heterogeneity (Fahrig et al., 2011; Vasseur et al., 2013). Therefore, it is essential to know which form of spatial heterogeneity actually affects ecological responses of the species of interest and at which scale(s) these effects occur; such insight would greatly improve our understanding of the spatial nature of animalenvironment relationships. However, spatial heterogeneity has seldom been partitioned in this way in practice.

Animals generally exhibit non-stationary patterns in habitat utilization and selection, which are explicitly shaped, for a large part, by the degree of spatial heterogeneity (structural and functional) occurring at different spatial scales (Boyce, 2006; Guisan et al., 2006). To understand these patterns in a heterogeneous landscape, one must be able to accurately link them to structural and functional components of the landscape (Wu et al., 2000). However, there are two critical limitations in determining the spatial nature of the associations between animals and spatial heterogeneity. First, we often have no a priori knowledge about the spatial scale(s) at which animals respond most strongly to spatial heterogeneity of the landscape (termed 'characteristic scale' -- de Knegt et al., 2010). Drawing ecological inferences about habitat selection/use based on investigations at other scales may result in misidentification of important ecological factors as well as of underlying 
mechanisms relevant to the species of interest (Wiens, 1989; Bradshaw \& Spies, 1992; Boyce et al., 2003; Reding et al., 2013). Second, the characteristic scale of the species' response, if it exists, does not have to coincide with the dominant scale of the landscape context considered (Murwira \& Skidmore, 2005; de Knegt et al., 2010; Pittiglio et al., 2013). To avoid mismatch between the scale analysed and the scale at which apparent effects of spatial heterogeneity (structural or functional) occurs, it is essential to couple ecological processes with spatial heterogeneity across multiple spatial scales (Wheatley \& Johnson, 2009; de Knegt et al., 2010). Yet direct assessment of scale-specific relationships between variables has remained a challenging problem in ecology, which hampers the explicit separation of the effects of spatial heterogeneity across scales (Keitt \& Urban, 2005).

In recent years, wavelet analysis has proven to be a promising tool to quantify spatial structure as a function of both scale and position (Murwira \& Skidmore, 2005; Carl \& Kühn, 2008; Cazelles et al., 2008). Wavelet analysis transforms spatial data into a series of coefficients that describe scalespecific variation in the data and thus provide information about the intensity of pattern at a particular location at different scales (Csillag \& Kabos, 2002; Keitt \& Urban, 2005). More importantly, the wavelet transform is a linear operator, making it possible to assess correlations between multiple spatial variables through traditional statistical analysis (e.g., linear regression) of the wavelet coefficients at each scale (Keitt \& Urban, 2005; Carl \& Kühn, 2008). For instance, Keitt and Urban (2005) applied wavelet transforms to multiple linear regressions (termed "wavelet-coefficient regression") to explore scalespecific patterns in vegetation growth, and found that different environmental variables show up as good predictors at different spatial scales. This approach differs from traditional methods in that both dependent and independent variables are wavelet-transformed prior to regression analysis. One apparent advantage of wavelet-coefficient regression is that it measures how a change in one factor at a given scale influences change in another factor at the same scale. Despite being successfully used to assess scalespecific vegetation-environment relationships (Keitt \& Urban, 2005; Carl \& Kühn, 2008), wavelet-coefficient regression, to our knowledge, has not been used to relate animal space use to resource attributes. As wavelet transform and wavelet-coefficient regression can efficiently characterise scale-specific patterns in spatial data (see Keitt \& Urban, 2005), it may provide critical clues to understanding the ecological mechanisms underlying animal habitat use in heterogeneous landscapes.

We present a wavelet-based approach to explore whether animals display differential responses to different forms of spatial heterogeneity and whether the responses are scale-specific in space. We used wavelet transforms to extract scale-specific information from animal space use and different 
landscape variables, then employed mixed-effects resource utilization functions (RUFs) to detect scale-specific associations in the transformed data. We illustrated this approach by testing whether structural and functional spatial heterogeneity of the landscape have differential and scale-specific influences on habitat use by American black bears (Ursus americanus; hereafter black bears) in the Massif de la Jacques-Cartier area in Canada. Black bears are a good candidate for examining scale-specific space use in relation to different landscape attributes because they have large home range areas and feed on a wide range of vegetation (Boileau et al., 1994). As a case study, we focused specifically on the following questions: (1) What form of landscape heterogeneity is associated with spatial patterns of habitat use by black bears? (2) At which spatial scale(s) do these associations occur? (3) Are these associations correlated among different spatial scales?

\subsection{Materials and methods}

\subsubsection{Study area}

The study area is located in the Massif de la Jacques-Cartier region ( $\sim 8900$ $\mathrm{km}^{2}$ ), Québec, Canada $\left(47^{\circ} 34^{\prime} \mathrm{N}, 71^{\circ} 12^{\prime} \mathrm{W}\right)$. The landscape has been heavily modified by intensive logging and rejuvenating boreal forests over the past 30 years (Brodeur et al., 2008). The forest cover is dominated by balsam fir (Abies balsamea), black spruce (Picea mariana), and paper birch (Betula papyrifera). Trembling aspen (Populus tremuloides), yellow birch ( $B$. alleghaniensis), and maples (Acer spp.) occur at low altitude areas (Boileau et al., 1994). Climate is relatively cold for this latitude, with an average of 75 days without frost. Depending on local weather, black bears in this area can den from November to mid-May (Brodeur et al., 2008).

\subsubsection{Black bear telemetry data}

Telemetry data used in our analysis was collected from a total of nine black bears equipped with GPS radio-collars, scheduled to record a location every 3 hours between May and September in 2005 and 2006 (Bastille-Rousseau et al., 2011). Among them, five males were monitored in 2005, four females were monitored in 2006, and one male was recorded in both 2005 and 2006 (see Supplementary material Appendix 1 for characteristics of individual bears). For the one animal monitored in both years, we analysed locations in each year separately. To improve positional precision in the analysis, locations with a dilution of precision (DOP) $>5$ were discarded (Dussault et al., 2001; Lewis et al., 2007). Locations with 3-D fix status made up 43 percent of the remaining GPS data set. 


\subsubsection{Estimating spatial intensity of habitat use for black bears}

For each black bear, the spatial intensity of habitat use within the home range, or utilization distribution (UD; Worton, 1989), was estimated by using a dynamic Brownian bridge movement model (dBBMM) introduced by Kranstauber et al (2012). The dBBMM is an improvement to the Brownian bridge movement model (BBMM; Horne et al., 2007). Both models improve on the traditional UD statistics (e.g., kernel density function; Silverman, 1986) by incorporating both the order of locations and the amount of time between them, in which the primary parameter is the estimated Brownian motion variance $\left(\sigma_{m}^{2}\right)$ that measures how irregular the path of an animal is between successive locations (Horne et al., 2007). However, the BBMM assumes a constant $\sigma_{\mathrm{m}}^{2}$ along an entire movement path, despite the fact that animal movement is actually composed of a number of behaviourally distinct movement patterns (e.g., Morales et al., 2004; Jonsen et al., 2005; Gurarie et al., 2009). The dBBMM extended the BBMM by allowing the estimated $\sigma_{\mathrm{m}}^{2}$ to vary according to changes in the movement behaviour of the animal along a trajectory, thereby providing a more accurate estimation of the UD for behaviourally heterogeneous animal tracks (Kranstauber et al., 2012). Briefly, the dBBMM estimates $\sigma_{\mathrm{m}}^{2}$ through moving a sliding window encompassing a number of locations along a path. Within each window, $\sigma_{\mathrm{m}}^{2}$ is first estimated via maximum likelihood for the whole path in the window, then the path is iteratively split into two parts at each location within the window, and $\sigma_{\mathrm{m}}^{2}$ estimated for each of the two parts. If the model consisting of a single $\sigma_{\mathrm{m}}^{2}$ has the lowest Bayesian information criterion (BIC) value, that $\sigma_{\mathrm{m}}^{2}$ is assigned to all steps within the window. If a model consisting of two $\sigma_{\mathrm{m}}^{2}$ values has the lowest BIC value, then that is considered evidence of a behavioural change, and the appropriate $\sigma_{\mathrm{m}}^{2}$ values are assigned to the steps on each side of the change point (Kranstauber et al., 2012). After a $\sigma_{\mathrm{m}}^{2}$ value has been estimated for each step, UDs can be estimated for any portion of the track as described by Horne et al. (2007). Because $\sigma_{\mathrm{m}}^{2}$ is estimated using the leave-one-out method, the window size needs to be defined to encompass an odd number of locations. In addition, a margin of greater than 3 locations on each end of the window in which no break points can occur must be specified. For more information see Horne et al. (2007) and Kranstauber et al. (2012).

To fit the dBBMM to the black bear tracks, we used a moving window size of 37 locations with a margin of 11 locations (equivalent to a window length of 6 days). We chose this window size based on the temporal resolution of each track (3-hour) and a priori assumption and knowledge of the time-scale of major behavioural shifts, as recommended by Kranstauber et al. (2012). We mapped the UD raster layers at $100-\mathrm{m}$ resolution. Note that using finer pixel 
sizes could improve the ability to capture subtle variation in spatial pattern of UD, but would greatly increase the computational time of UD estimation and subsequent wavelet analysis. Home ranges for each of the black bear were delineated by the $95 \%$ contours of their respective UD, and the overlaps between the home ranges of same sex groups were generally low $(<30 \%)$. The UD calculations were implemented using the 'move' package (Kranstauber \& Smolla, 2013) in the R software environment (version 2.15.2; R Development Core Team, 2011).

\subsubsection{Measuring landscape spatial heterogeneity}

Our analysis was focused specifically on structural and functional spatial heterogeneity inside the home ranges of black bears. Both forms of landscape heterogeneity were measured from an existing land cover map (Table 1), which was based on classified Landsat Thematic Mapper imagery (taken in 1999; 25-m resolution) and updated for recent cut-blocks and roads in the study area (Bastille-Rousseau et al., 2011). Many existing landscape metrics (patch aggregation, edge density, etc.) can be used to measure the structural properties of landscape heterogeneity. For the purpose of illustration, we chose Simpson's Diversity index (SIDI; McGarigal \& Marks, 1995) to quantify structural spatial heterogeneity because this metric takes into account both the number of cover types present and the abundance of each cover type (McGarigal \& Marks, 1995). Structural heterogeneity was produced by calculating the SIDI of cover types through a $1 \mathrm{~km} \times 1 \mathrm{~km}$ moving window, resulting in a map of land cover diversity per unit (i.e., $1 \mathrm{~km}^{2}$ ). Functional landscape heterogeneity was delineated by a map of forage abundance per unit for black bears using the same moving window approach, in which the forage abundance value was calculated based on relative forage abundance index for each cover type (Bastille-Rousseau et al., 2011; also see Appendix 2 for further information).

In addition to spatial patterns of the structural and functional landscape heterogeneity in the study area, we examined the potential effects of different vegetation age-classes on patterns of space use by black bears. By doing so, we aimed at increasing our understanding of the mechanisms behind the spatial patterns of habitat use by black bears in intensively modified landscapes. We grouped eight vegetation types into five ageclasses: mature forest, young forest, regenerating forest, clearcut, and shrubland (Table 2). We calculated the proportion of each vegetation ageclass per unit by moving a $1 \mathrm{~km} \times 1 \mathrm{~km}$ window across the study area. This produced five separate layers that reflect spatial patterns of particular vegetation age-classes in post-logging forests. All landscape computations were carried out in the program FRAGSTATS 4.2 (McGarigal \& Marks, 1995). 
Prior to the landscape computation, the grain size of the land cover map was coarsened to match the 100-m resolution of UD layers for black bears.

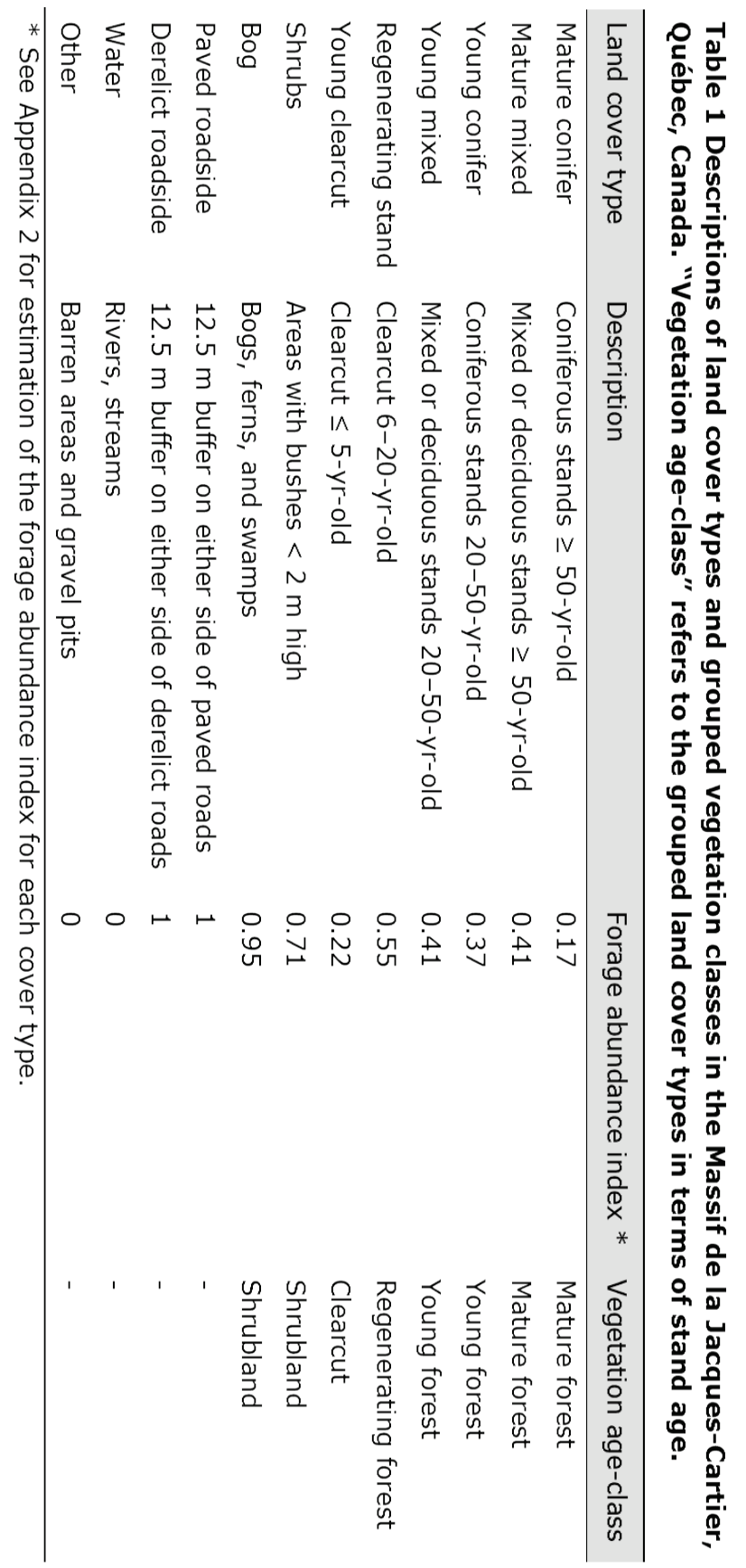


Table 2 Descriptions for landscape variables that were related to habitat use by American black bears in the Massif de la Jacques-Cartier, Québec, Canada.

\begin{tabular}{|c|c|}
\hline Variable name & Description \\
\hline $\begin{array}{l}\text { Simpson's } \\
\text { Diversity index of } \\
\text { cover types } \\
\text { (SIDI) }\end{array}$ & $\begin{array}{l}\text { Measure of structural landscape heterogeneity that } \\
\text { equals } 1 \text { minus the sum, across all land cover types, of } \\
\text { the proportional abundance of each cover type squared. } \\
\text { The value of SIDI represents the probability that any } 2 \\
\text { pixels selected at random would be different cover types. } \\
\text { SIDI = } 0 \text { when the landscape contains only } 1 \text { cover type } \\
\text { (i.e., no diversity), while SIDI approaches } 1 \text { as the } \\
\text { number of different cover types increases and the } \\
\text { proportional distribution of area among cover types } \\
\text { becomes more equitable. }\end{array}$ \\
\hline $\begin{array}{l}\text { Relative forage } \\
\text { abundance } \\
\text { (FORAGE) }\end{array}$ & $\begin{array}{l}\text { Measure of functional landscape heterogeneity that is } \\
\text { calculated as the average of the forage abundance index } \\
\text { values for all cover types within a } 1 \mathrm{~km} \times 1 \mathrm{~km} \text { moving } \\
\text { window and weighted by their respective areas in that } \\
\text { window. The value of FORAGE ( } 0-1 \text { scale) reflects relative } \\
\text { availability of food resources for black bear. }\end{array}$ \\
\hline$\%$ Mature & Proportion of mature forest per $\mathrm{km}^{2}$ \\
\hline$\%$ Young & Proportion of young forest per $\mathrm{km}^{2}$ \\
\hline$\%$ Regenerating & Proportion of regenerating forest per $\mathrm{km}^{2}$ \\
\hline$\%$ Clearcut & Proportion of clearcut per $\mathrm{km}^{2}$ \\
\hline$\%$ Shrub & Proportion of shrubland per $\mathrm{km}^{2}$ \\
\hline
\end{tabular}

\subsubsection{The wavelet transform analysis}

Wavelet transform analysis can be used to detect scale-specific patterns in spatial transacts or two-dimensional images by decomposing signal variance into separate components at different scales (Daubechies, 1992; Dale \& Mah, 1998; Cazelles et al., 2008). Here we provide a brief overview of the wavelet transform. Further details of the wavelet transform and its application can be found in Daubechies (1992), Torrence and Compo (1998), and Cazelles et al. (2008).

The wavelet transform is basically a linear filter which evaluates the degree of similarity between a wavelet template and the structure of an image at each location, and this comparison is repeated at all locations for multiple template sizes, thereby decomposing the original image into wavelet transform coefficients that describe respective variations at different scales 
(Figure 1 ; Torrence \& Compo, 1998). The wavelet template $\varphi(x)$ (also termed "mother wavelet") is a scalable windowing function whose integral over all $x$ equals zero. When the template fits the observed data well, the value of the wavelet transform coefficient at that position is high; when it does not, the value is low (Dale \& Mah, 1998; Csillag \& Kabos, 2002). The size of the wavelet template determines the scale of pattern captured and, in the discrete context (i.e., the discrete wavelet transform; hereafter DWT), increases at successive bases $\left(2^{l}\right.$, with $\left.l=1,2, \ldots L\right)$ in terms of pixels. At each level of decomposition $l$, the wavelet transform calculates two sorts of coefficients: the 'smooth' and the 'details' in the horizontal, vertical, and diagonal directions, where the 'smooth' part is an averaged version of the original data, and the 'details' describe the deviances from the average value of the data at each direction and scale (Bruce \& Gao, 1996). Thus, the wavelet approximation of an image $F(x, y)$ is the sum of the smooth and detail functions at different scales:

$$
\hat{F}(x, y)=\sum_{l=1}^{L} \sum_{k} d_{l}^{k}(x, y)+s_{L}(x, y)
$$

eqn. 1

where $d_{l}^{k}$ represents the directional detail coefficients and $s_{L}$ the smooth coefficients. Each detail coefficient is associated with a particular level $l$, a direction $k$, and a particular location. Therefore, changes in pattern of $F(x, y)$ are captured in detail coefficients for different levels $l=1,2, \ldots L$, and those not included in detail coefficients are mapped in smooth coefficients of last level $L$ (Figure 1). To avoid any confusion, we note that here the term "scale" refers to the different sizes of the wavelet template, and the term "level" refers to the separate sets of coefficients in wavelet decomposition. The relationship between the level and scale is quite straightforward in that a level of $l$ corresponds to a scale of $2^{l} \times a$, where $a$ is the pixel size of the original image.

In this study, a two-dimensional maximum overlap discrete wavelet transform (2D MODWT; Percival \& Walden, 2000) was employed to decompose both UD and landscape covariates into scale-specific wavelet coefficients. The MODWT, also known as the "stationary wavelet transform", is distinct from the standard DWT in that the resulting levels of the decomposition are not orthogonal (Percival \& Walden, 2000). Unlike the DWT that each level of the resulting decomposition contains $\left(2^{\log 2(N)-l}\right)^{2}$ coefficients ( $N$ is the number of pixels in the original image), the number of coefficients at each level of the MODWT decomposition is equal to the original dimensions of the data, allowing one to produce meaningful images of the separate levels of the decomposition (James et al., 2011). Moreover, the MODWT can assess global and local wavelet variance more robustly, and is 
less affected by differences in the wavelet template than the DWT (Percival \& Walden, 2000). We performed the 2D MODWT in the R package "waveslim" (Whitcher, 2012). The Daubechies 4 template was used in the wavelet transform because this template is effective in identifying peaks of variations in spatial data (Daubechies, 1992). Given the mean home range extent of the black bears in this area (averaged $65 \mathrm{~km} 2$; Brodeur et al., 2008), we stopped the wavelet transform at level 5 (Figure 2). Levels 1-5 correspond to wavelet template sizes of $2,4,8,16$, and 32 pixels, respectively, or scales $200-\mathrm{m}$, $400-\mathrm{m}, 800-\mathrm{m}, 1600-\mathrm{m}$, and $3200-\mathrm{m}$, respectively, so using a larger template size would by virtue of redundancy prohibit effective detection of variation within home range areas. Prior to the wavelet transformation, all spatial data were normalized to a mean of zero and unit variance, and each dimension (row and column) was padded with zeroes to the next highest power of 2 .

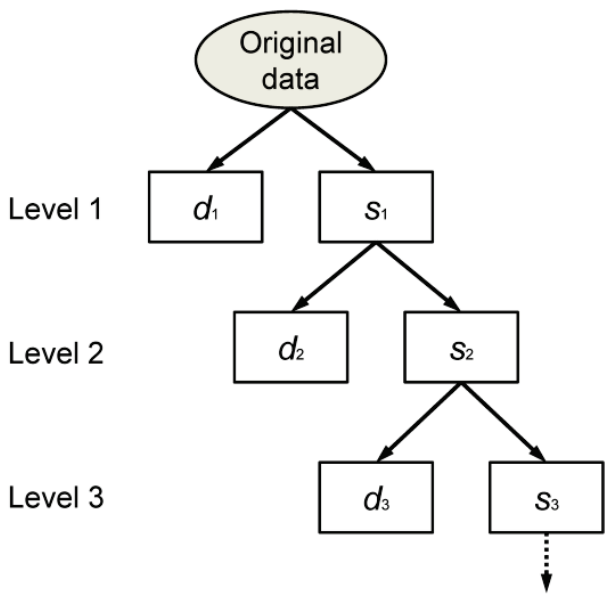

Figure 1 Graphical depiction of wavelet transform. In wavelet analysis, the original data are split into smooth and detail parts (denoted by $s$ and $d$, respectively). The smooth is then itself split into a second-level smooth and detail, and the process is repeated so that the data are broken down into many scale-specific components.

After wavelet transformation, the detail coefficients of UD and landscape covariates (hereafter referred to as "transformed UD" and "transformed covariates", respectively) at each level of the decomposition were extracted within the home range of each individual bear and used in subsequent analyses. We examined Spearman correlation coefficients between each pair of transformed covariates, and the results indicated that the correlations were generally low $(|\rho|<0.5)$. 
(a)

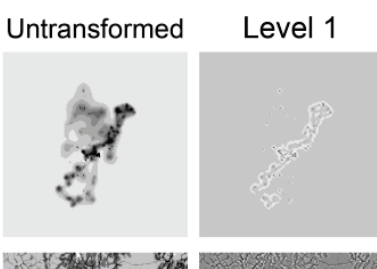

(b)

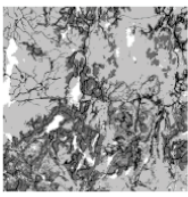

(c)

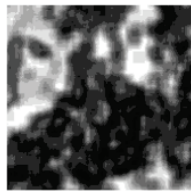

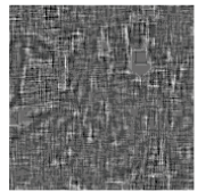

Level 2
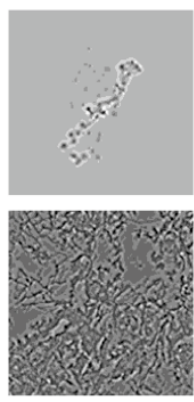

Level 3
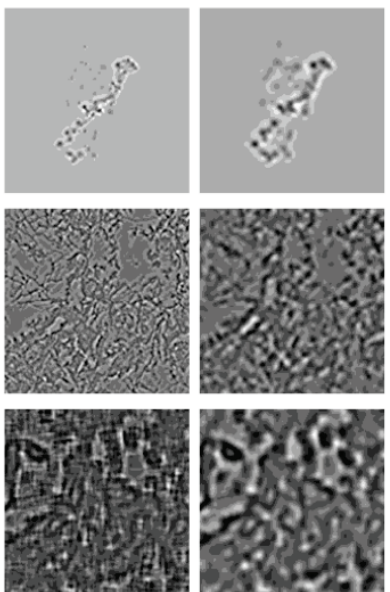

Level 4

Level 5
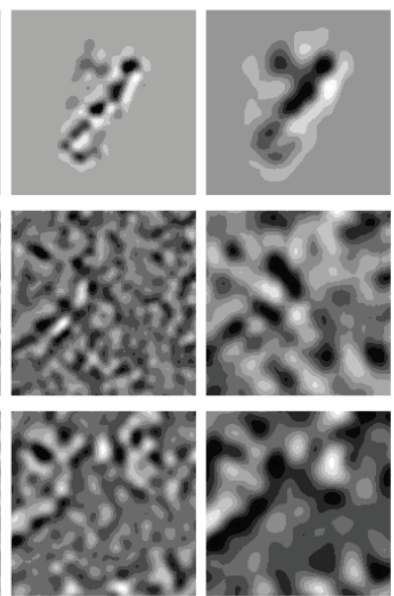

Figure 2 Illustrations of wavelet decompositions of (a) pattern of black bear's UD, (b) local forage abundance for black bears, and (c) SIDI of cover types. Levels 1 - 5 correspond to spatial scales of 200-m, 400-m, 800-m, 1600-m, and $3200-\mathrm{m}$, respectively.

\subsubsection{Relating black bear's UD to landscape heterogeneity}

We first examined wavelet covariance between transformed UD and transformed landscape covariates to explore their scale-specific associations. Wavelet covariance, namely multiscale analysis of covariance, is an exploratory tool to assess associations between variables on a scale by scale basis (Whitcher et al., 2000). Given two sequences $A$ and $B$, the wavelet covariance is

$$
\operatorname{cov}_{l}(A, B)=\left\langle d_{l}(A) \times d_{l}(B)\right\rangle
$$

eqn. 2

where \langle\rangle indicates summation and $l$ is the level of the decomposition (Keitt \& Urban, 2005). Strong positive covariance between wavelet coefficients indicates a positive association at a particular scale. This approach can also be applied to spatially formed variables. In such cases, we need to convert these spatially formed data into vectors for covariance computation. Our analysis of wavelet covariance was conducted for each individual bear and summarized separately for males and females.

To further characterize the correlation structure between patterns in UD of black bears and patterns in the landscape covariates, we evaluated scalespecific resource utilization functions (RUFs; Marzluff et al., 2004) through a wavelet-coefficient regression approach (Keitt \& Urban, 2005; see Supplementary material Appendix 4 for further detail). We fitted a multiple linear-regression model to transformed UD and transformed landscape 
covariates at each level of the decomposition, with the intercept forced through the origin (i.e., $\beta_{0}=0$ ). The transformed UD was used as the dependent variable, and the transformed landscape covariates were treated as independent variables. Because black bears exhibit apparent sexual differences in their ranging behaviour (Powell et al., 1997), we included a random coefficient for each landscape covariate to account for differences in the response between sex groups. The form for the linear mixed-effects model for location $i$ and sex group $j$ is:

$$
y_{i j}=\left(\beta_{1}+\gamma_{1 j}\right) x_{1 i j}+\cdots+\varepsilon_{i j}
$$

eqn. 3

where $\beta_{1}$ is the fixed-effect resource utilization coefficients for landscape covariate $x_{1 i j}, \gamma_{1 j}$ is the variances around $\beta_{1}$ between sex groups for landscape covariate $x_{1 i j}$, and $\varepsilon_{i j}$ is unexplained residual variation. Such mixed-effects models allow RUF inference at both the population-level and sex-level by accounting for unbalanced sample designs and autocorrelation (Gillies et al., 2006; Schreinemachers \& Berger, 2011).

Model selection was conducted hierarchically (Ten Have et al., 1999). Firstly, we employed the second-order Akaike information criterion adjusted for small sample size (AICc; Burnham \& Anderson, 2002) to select the most parsimonious fixed-effects models from the candidate habitat covariates measured at each spatial scale. We then used all of the covariates included in each of the top models for each spatial scale to build a full fixed-effects model representing habitat use at all scales. We considered models with differences of AICc ( $\triangle \mathrm{AICc}$ ) values $\leq 2$ from the best-fit model equally parsimonious (Burnham \& Anderson, 2002), thereby including variables from all such models for further mixed-effects analysis (see Appendix 5 for results of model selection). Model selection for models with random effects is complicated because the intended scope of inference influences the derivation of information theoretic metrics such as the AIC (Gillies et al., 2006); herein, we did not give further consideration to model selection for random effects.

We considered two model specifications to assess the scale-specific response of black bears in relation to different forms of landscape spatial heterogeneity. The first scale-specific mixed-effects RUFs was built on the two heterogeneity metrics (Simpson's diversity and forage abundance), aiming at understanding how structural and functional landscape heterogeneity affect space use patterns of black bears and at which scale(s) these effects occur. The second scale-specific mixed-effects RUFs was built on local proportions of the five vegetation age-classes, with the aim of identifying at which spatial scale(s) the influences of particular age-classes on black bears' space use patterns occur. This helps to increase our 
understanding of the mechanisms of black bears' space use in relation to spatial heterogeneity affected by anthropogenic disturbances. In the latter model, interactions between covariates were excluded because of computational (convergence) difficulties with large data sets in mixed-effects modelling approach. To compare our wavelet-transformed RUFs with traditional modelling approaches, we also built mixed-effects RUFs of untransformed data. This is equivalent to assessing the effects of spatial variations averaged over different scales. All mixed-effects RUFs were fitted by maximum likelihood using the "Ime4" package (Bates \& Maechler, 2009) in the statistical software $R$, version 2.15.2 (R Development Core Team, 2011).

\subsection{Results}

Male and female black bears exhibited distinct patterns of wavelet covariance between the transformed UD and relative forage abundance (hereafter referred to as "FORAGE") at different scales (Fig. 3a). For females, the transformed UD positively covaried with the transformed FORAGE at levels 2 and 3 (400-m and $800-\mathrm{m}$ scales, respectively), but exhibited negative covariance at level 5 (3200-m scale; Fig. 3a). In contrast, the transformed UD of males showed positive association with the transformed FORAGE at levels 4 and 5 (1600-m and 3200-m scales, respectively; Fig. 3a), indicating that the spatial pattern of food abundance may influence the probability of use by males only at broad scales. The wavelet covariance between the transformed Simpson's diversity index of cover types (SIDI) and transformed UD was generally weak for both males and females (Fig. 3b).

When relating the transformed UD to proportions of vegetation age-classes, differential patterns emerged in the wavelet covariance depending on the age-classes compared (Fig. 3c-g). For instance, the transformed UD of females showed positive association with the transformed proportion of mature forests at level 5 (3200-m scale; Fig. 3c), but negatively covaried with the transformed proportion of regenerating forests at the same level (3200-m scale; Fig. 3e). Interestingly, males and females exhibited contrasting patterns in the wavelet covariance between UD and proportion of shrubland at levels 4 and 5 (1600-m and 3200-m scales, respectively), in which the proportion of shrubland covaried positively with the UDs of males but associated negatively with the UD of females (Fig. 3g). 
(a)

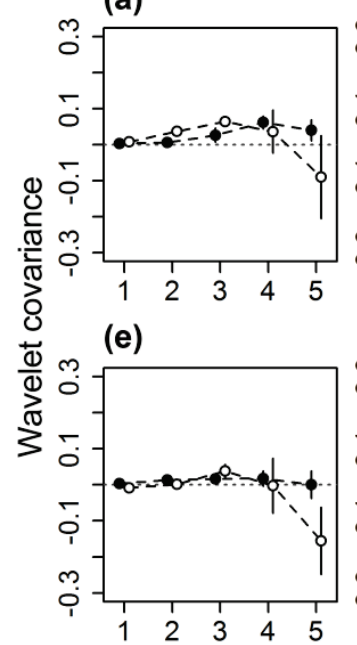

(b)

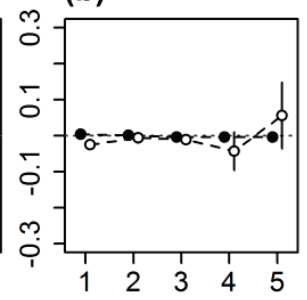

(f)

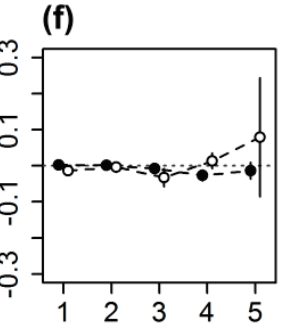

(c)

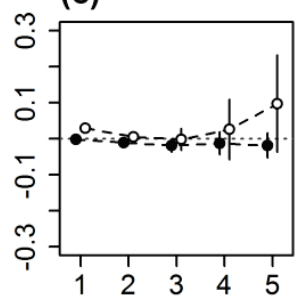

(g) (d)
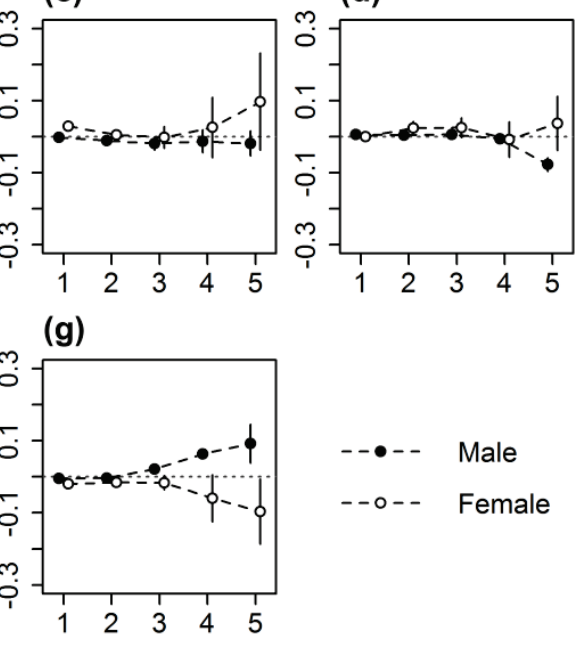

$--\bullet-$ Male

--o-- Female

Figure 3 Wavelet covariances (mean \pm SE) of UDs of male and female black bears with landscape covariates (a) FORAGE, (b) SIDI, (c) \% Mature, (d) \% Young, (e) \% Regenerating, (f) \% Clearcut, and (g) \% Shrubland as a function of spatial scale. Levels 1 - 5 correspond to spatial scales of 200-m, 400-m, 800-m, 1600-m, and 3200-m, respectively. Dotted lines indicate zero covariance. See Table 2 for definition of each landscape covariate.

To further explore how spatial patterns of structural and functional spatial heterogeneity shaped the UDs of black bears and how these relationships varied between sex groups, we fitted mixed-effects RUFs to the wavelet coefficients at levels $1-5$, as well as to the untransformed data. For the untransformed data, we found that only SIDI (i.e., structural heterogeneity) had a significant positive effect on the UDs of black bears $(P<0.05)$, while FORAGE (i.e., functional heterogeneity) and their interaction had weak influences on space use patterns of bears inside home ranges (Table 3 ). However, when the data were wavelet transformed, the scale-specific RUFs highlighted distinct associations between UDs and different forms of spatial heterogeneity at different scales (Table 3 ). For example, FORAGE was found to have significant effects on the UDs of black bears at levels 3 and $4(800-\mathrm{m}$ and 1600-m scales, respectively), whereas the effect of SIDI were no longer significant at all levels investigated (Table 3). The interaction between SIDI and FORAGE significantly influenced the UDs of black bears at levels 2-4 (400-m, 800-m, and 1600-m scales, respectively). Furthermore, our scalespecific RUFs showed quite different correlation structure between male and female bears at different scales. For instance, forage abundance generally had positive effects on UDs of males at levels 4 and 5 (1600-m and 3200-m, respectively), but affected UDs of females mainly on level 3 and 4 (800-m and 1600-m, respectively; Fig.4a). Covariate SIDI had negative effects on 
the UDs of female bears at level 1 (200-m scale), but imposed little effects on the patterns of space use by males across all scales (Fig. 4b). A similar pattern was found for the effects of the interaction between FORAGE and SIDI (Fig. 4c).
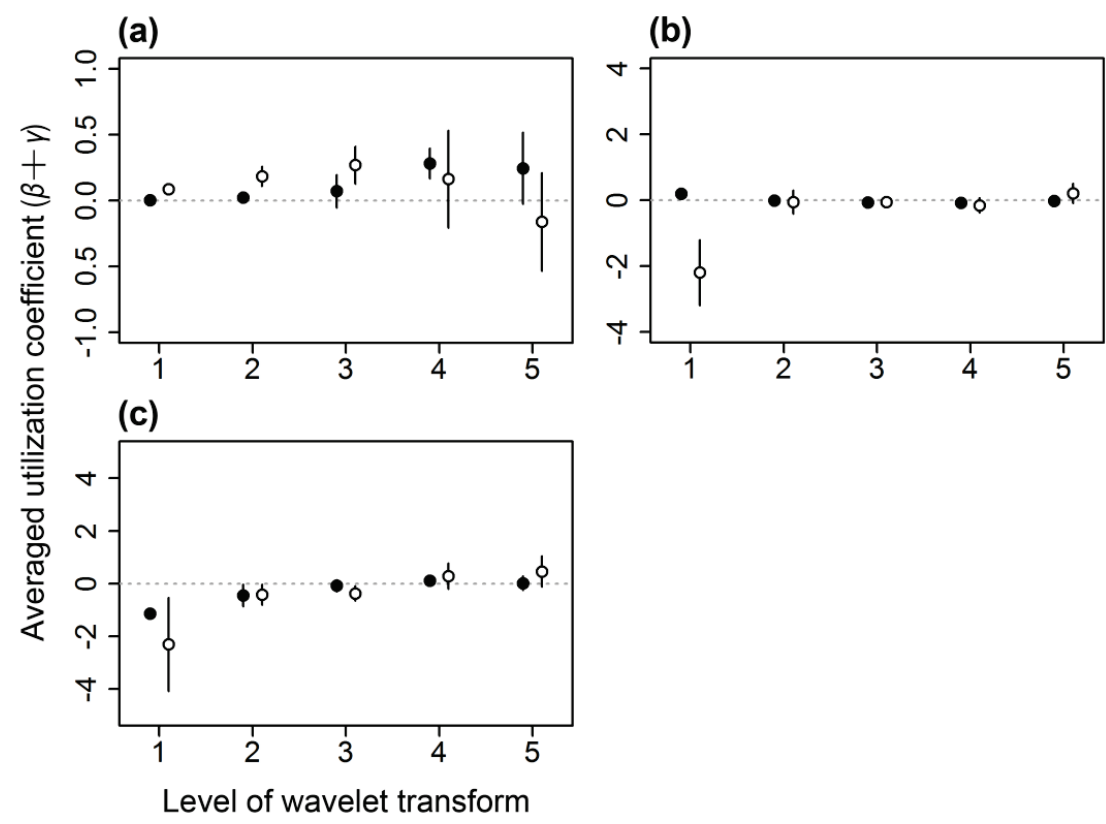

Figure 4 Estimates of scale-specific utilization coefficients (mean \pm SE) based on landscape covariates (a) FORAGE, (b) SIDI, and (c) their interaction in terms of sex groups of black bears. Levels 1 - 5 correspond to spatial scales of 200-m, 400-m, 800-m, 1600-m, and 3200-m, respectively. Black dots represent males and white dots females.

When focusing specifically on the effects of local proportion of the five vegetation age-classes, the RUFs of the untransformed data showed that the proportion of shrubland had non-significant influence on space use by black bears, contrasting with the scale-specific RUFs that the effects of the proportion of shrubland were significant at level 3 and 5 (800-m and 3200-m scales, respectively; Table 4). The other four vegetation age-classes (mature forest, young forest, regenerating forest, and clearcut) also exhibited differential correlation with UDs of black bears at different scales (Table 4). The scale-specific RUFs further characterized that vegetation composition affecting the pattern of space use by females was primarily at local scales in nature (Fig. 5). For examples, the proportions of mature and young forests did not influence the UDs of females above level 3 (800-m scale; Fig. 5a, b). The proportion of regenerating forests strongly influenced the UDs of females positively at levels 2 and 3 (400-m and 800-m scales, respectively; Fig. 5c). The proportions of shrubland had a negative influence on the UDs of females 
only at level 1 (200-m scale, Fig. 5e). Interestingly, no vegetation ageclasses were found to have significant influences on the UDs of males at all levels of decomposition (Fig. 5).

(a)

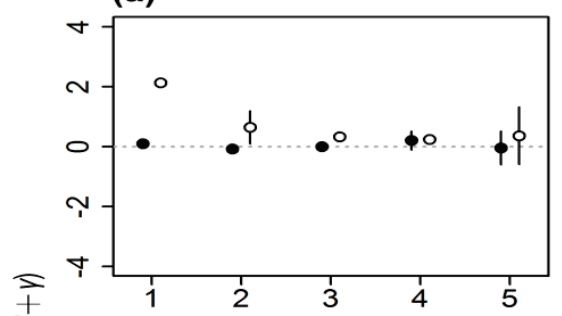

(c)

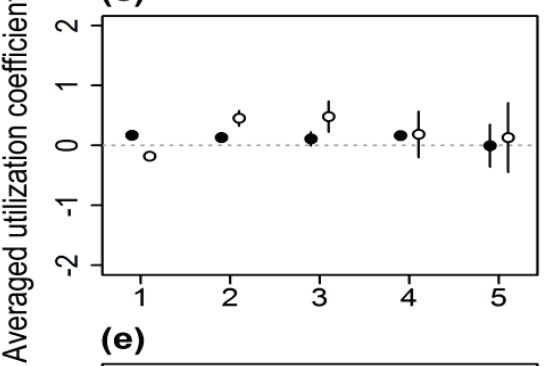

(e)

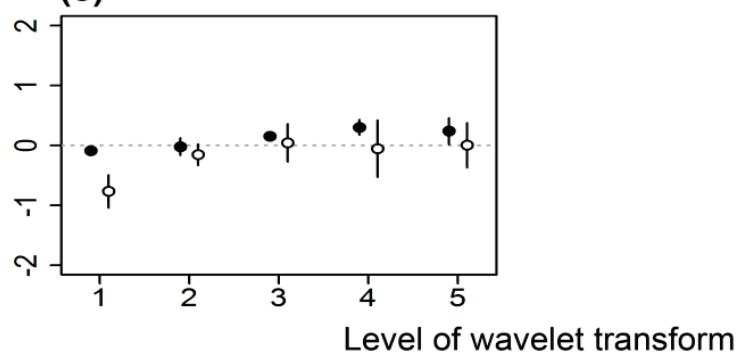

(b)

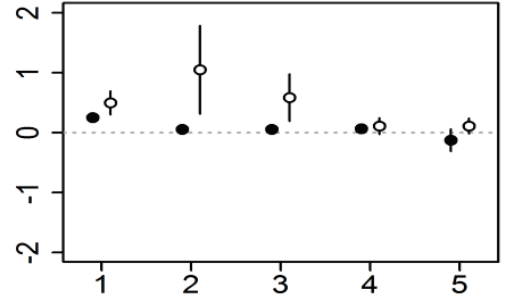

(d)

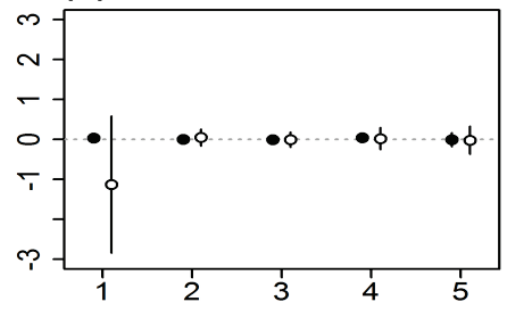

Figure 5 Estimates of scale-specific utilization coefficients (mean \pm SE) for local proportions of vegetation age-classes (a) \% Mature, (b) \% Young, (c) \% Regenerating, (d) \% Clearcut, and (e) \% Shrubland in terms of sex groups of black bears. Levels 1 - 5 correspond to spatial scales of 200-m, 400-m, 800$m, 1600-m$, and 3200-m, respectively. Black dots represent males and white dots females. 


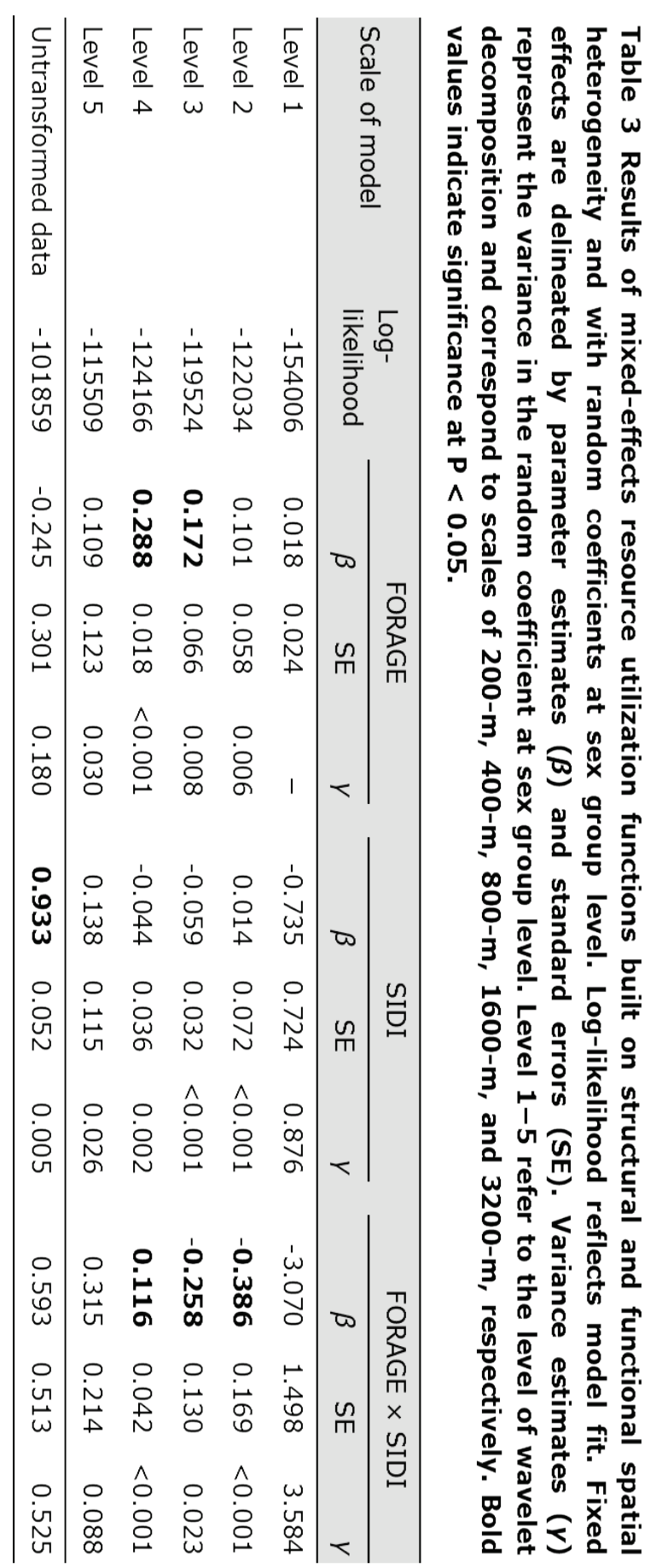




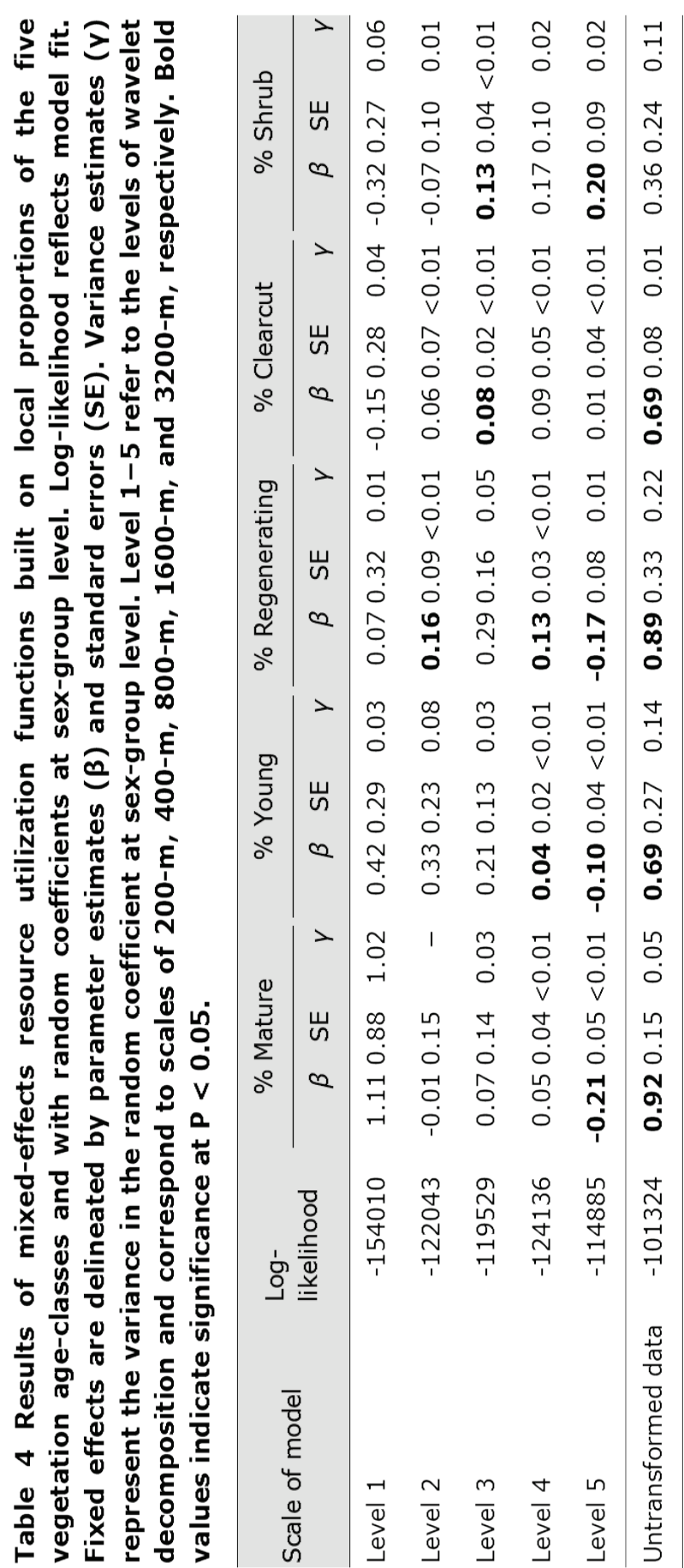




\subsection{Discussion}

We combined wavelet transform with mixed-effects resource utilization functions to extract scale-specific relationships between different forms of spatial heterogeneity and habitat use by American black bears within their home ranges. Our analysis demonstrates that the wavelet-based method provides an efficient way of analysing scale-specific patterns of animal space use in relation to spatial variations of landscape attributes. We found strong scale-specific differences in the effects of structural and functional spatial heterogeneity on the patterns of habitat use by black bears. For examples, FORAGE (functional heterogeneity) had significant effects at scales $800-\mathrm{m}$ and 1600-m, whereas SIDI (structural heterogeneity) alone did not significantly influence utilization distributions of black bears at all scales investigated. Such differences in the characteristic scales of the black bears' response to structural and functional heterogeneity suggest that the spatial scaling of animal-habitat relationships should consider not only the structural and functional properties of landscapes, but also the variation in scaledependent responses of animals to the functional heterogeneity in the landscape. We also found that black bears avoided mature coniferous forest at broad scales, concentrating on regenerating forest and shrubland at local scales. These results extended previous findings of Brodeur et al. (2008) and indicate that post-logging forest stands with high fruit density were important in shaping bears' habitat use at a variety of scales (Welch et al., 1997). Our scale-specific RUFs also showed that males and females varied considerably in their use of particular resources at different scales. For instance, female bears were more sensitive to local-scale spatial structure of regenerating forests and shrubs that provide abundant soft mast for black bears in boreal forests. These results indicate that sexual variability, when present, should not be ignored when making inferences about behaviour and scale at a population level (Bolnick et al., 2007).

To detect meaningful and significant spatial scales from non-stationary ecological data, the method being used should be capable of identifying both local and global attributes of spatial pattern (Humphries et al., 2013). Traditional multi-scale analyses of animal-habitat relationships have generally been conducted at arbitrarily selected scales around observation points (e.g., different searching radii), with little consideration of inherent landscape structure and its connection to the pattern of interest (e.g., Denoel \& Lehmann, 2006; Finlayson et al., 2008). However, these methods are generally confounded by spatial autocorrelation (Legendre, 1993). Our wavelet-based approach used wavelet-transformed response variable and predictor variables in model fitting, and correlations between variables were examined through regression analysis of the wavelet coefficients at each decomposing scale (Keitt \& Urban, 2005). In other words, we examined how 
a change in the pattern of one factor at a given scale influences change in patterns of other factors at the same scale, rather than just whether the absolute levels are correlated. Because wavelet transform decomposes the original data at successively broader scales (the power 2 scaling), there is no need to choose an arbitrary window size over which to search for correlations between variables. Furthermore, the wavelet coefficients at a given scale represent variation in the variable at that scale which are orthogonal to variation at other scales (Daubechies, 1992). Consequently, the analysis of the wavelet coefficients is likely to be more robust in direct assessment of scale-specific relationships with controls for confounding background noise and autocorrelation (Carl \& Kühn, 2008). From these perspectives, the wavelet-based method is promising and can be used in a wide range of ecological applications such as presence-absence analysis of species distribution (e.g., Carl \& Kühn, 2008) or resource selection analysis of useavailability data (Manly et al., 2002). For example, a species distribution may be controlled at fine scales by the presence of food resources within forage habitat, but at a broader scale, environmental patterns typically influence the location of home ranges. We expect that testing for scale-specific correlations using the wavelet-based approach will highlight important environmental drivers of species distributions at different scales.

There are a few limitations of the wavelet-based methods that need further clarification. First, there are a number of wavelets (e.g., the Haar wavelets and the Morlet wavelets) available for data transformation (Bradshaw \& Spies, 1992). Using different wavelet forms may produce different results in some sense as a consequence of the degree of similarity between the shape of the wavelet and the dominant pattern in the ecological data (Dale \& Mah, 1998). Therefore one should investigate whether the wavelets selected is effective in detecting scale-specific relationships of interest. Second, the resolution/grain size of the raster data determines the smallest applicable wavelet; that is, the wavelets cannot detect dominant patterns smaller than the resolution of the raster. Therefore, a finer pixel size allows one to capture fine landscape features that might be relevant to behaviours of animals. However, computation time is presently a limiting factor when analysing large raster datasets with fine resolution (as in our case, the transform of a single raster layer at 50-m grain size took $>10$ hours). On the other hand, a large grain size would effectively average out spatial heterogeneity occurring at finer resolution. Identifying the appropriate resolution of wavelet analysis requires a detailed understanding of a species' ecology. Furthermore, the size (scale) of wavelets in discrete wavelet transformation operates as a power of 2 , thereby providing stepwise information rather than in a continuous fashion. If the true correlation occurs at scales falling between the levels of decomposition, they may be assigned to the wrong scale (Keitt \& Urban, 2005). Using a continuous wavelet function (CWT; Daubechies, 1992; 
Torrence \& Compo, 1998) could be an option to compute the transform across a continuum of scales, but instead would then contain huge redundant information in the decompositions, thereby decreasing the effectiveness in detecting scale-specific patterns in two-dimensional ecological data (Keitt \& Urban, 2005). The implications for the trade-off between spatial independence and scale resolution in data analysis need to be further explored. Nevertheless, it is clear that scale-specific analysis using wavelets is a flexible mean in ecological applications concerned with multivariate, multi-scaled patterns. We encourage the use of the methodological framework outlined here in future animal studies.

\section{Acknowledgements}

We greatly thank Douglas A. Kelt and anonymous reviewers for their insightful remarks. This work was funded by the Erasmus Mundus External Co-operation Window (EMEWC) Programme of the European Union and cofunded by the ITC Research Fund. Fieldwork was supported by the Ministère du Développement durable, de l'Environnement, de la Faune et des Parcs du Québec, the Ministère des Transports du Québec, the Fonds pour le rétablissement des espèces en péril, and the Fondation de la faune du Québec. We thank R. Courtois, C. Dussault and J.-P. Ouellet for making the field component of this study possible, as well as R. Lemieux, M. Guilbeault, M. McCann, and V. Brodeur for the bear captures. 


\section{Appendix 1}

Description of the telemetry data of black bears used in the analysis.

\begin{tabular}{rrrrrrr}
\hline ID & Sex & Age & $\begin{array}{r}\text { Litter } \\
\text { size }\end{array}$ & Starting date & $\begin{array}{r}\text { Duration } \\
\text { (days) }\end{array}$ & $\begin{array}{r}\text { Number of GPS } \\
\text { locations }\end{array}$ \\
\hline 1 & F & 3.5 & 2 & 30 April 2006 & 158 & 612 \\
2 & F & 7 & 3 & 29 April 2006 & 91 & 340 \\
3 & F & 4 & 0 & 30 April 2006 & 124 & 410 \\
4 & F & 7.5 & 0 & 1 May 2006 & 178 & 880 \\
5 & M & 10 & - & 8 May 2005 & 159 & 670 \\
6 & M & 6.5 & - & 24 May 2005 & 126 & 424 \\
7 & M & 9.5 & - & 21 June 2005 & 113 & 515 \\
8 & M & 6.5 & - & 20 June 2005 & 94 & 466 \\
9 & M & 5.5 & - & 31 May 2005 & 137 & 650 \\
10 & M & 5.5 & - & 30 April 2006 & 115 & 462 \\
\hline
\end{tabular}




\section{Appendix 2}

\section{Estimation of relative forage abundance index for cover types}

The relative forage abundance index for American black bears were estimated based on vegetation survey conducted in June. Survey plots were distributed randomly across the study area, among land cover types, and within 50 to $500 \mathrm{~m}$ from paved or derelict roads. Vegetation surveyed included only items generally found in the diet of black bears during spring, which included forbs, graminoids, and tree leaves (Boileau et al. 1994, Samson 1995). At all survey locations, the percent cover of forbs and graminoid plants in four $1-\mathrm{m}^{2}$ quadrats located $25 \mathrm{~m}$ apart in each cardinal direction were recorded and then converted into biomass using allometric equations developed by Turner et al. (2004). At the same locations, all deciduous trees and shrubs $1 \mathrm{~m}$ tall in a $20-\mathrm{m}^{2}$ quadrat were also recorded as an index of leaf and bud abundance. A relative index of vegetation abundance for each land cover type was then calculated. The abundance of the different vegetation classes was weighted according to their proportion in the spring diet of black bears in Québec, as described by Boileau et al. (1994) and Samson (1995). Separate indices were estimated based on weighing factors provided by each of the 2 studies and then calculated an average index for each land cover type. A map of relative vegetation abundance (0-1 scale) was then created by considering the average vegetation index associated with each of the 12 land cover types. 


\section{Appendix 3}

Illustrations of the landscape variables used in the analysis: Simpson's diversity of cover types, forage abundance, and local proportion of mature forest, young forest, regenerating forest, clearcut and shrubland. The variables are mapped at 100 meters resolution.
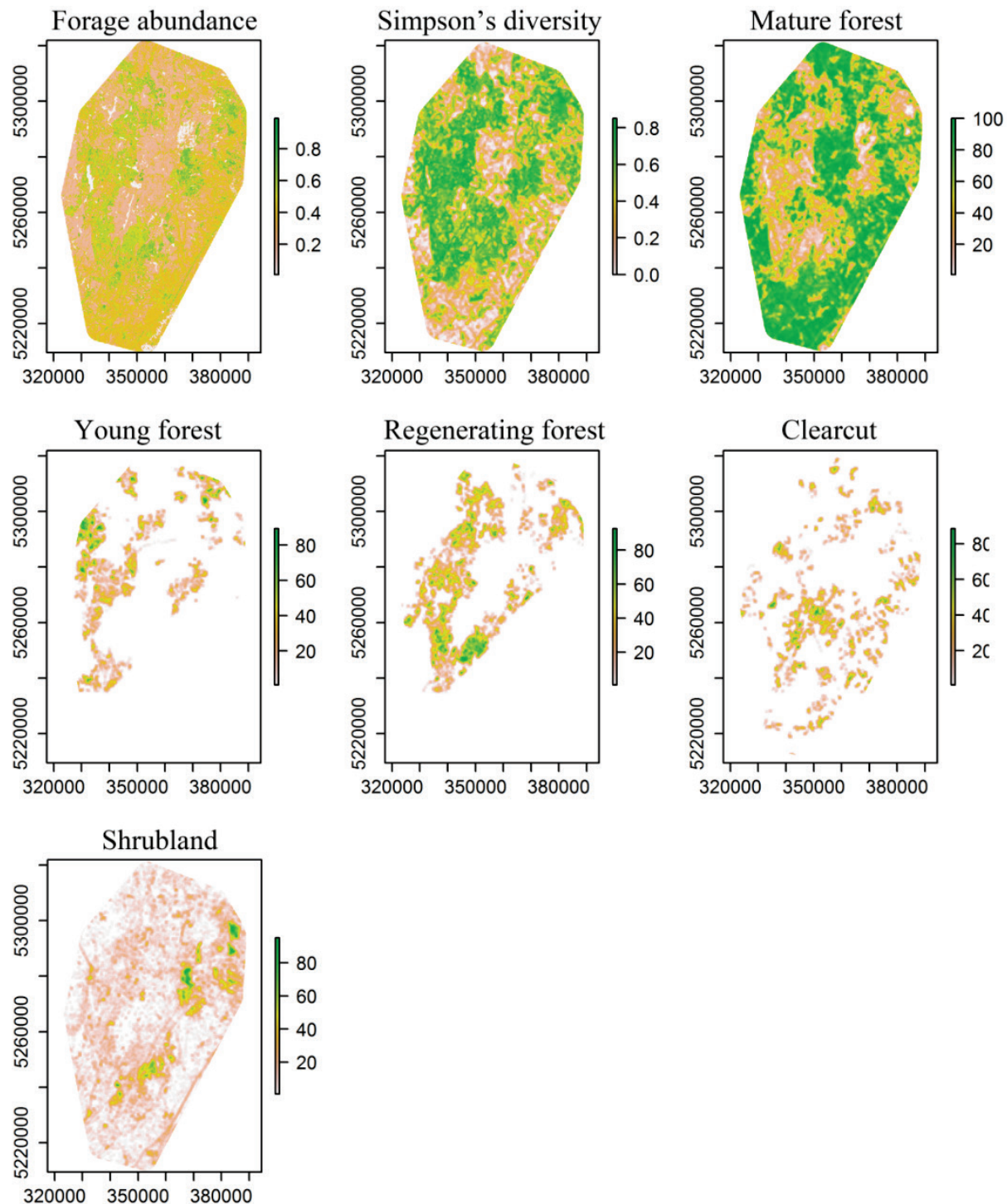


\section{Appendix 4}

\section{Brief description of wavelet-coefficient regression}

Wavelet-coefficient regression is, by definition, the multiple regression of wavelet-transformed data. If we consider the case that a wavelet transform is applied to the normal linear model, we can expect that the regression parameters will remain unchanged. This is due to the fact that the wavelet transform is a linear operator. Thus, the standard multiple linear model:

$$
y=\beta_{0}+\beta_{1} x_{1} \ldots+\varepsilon
$$

can be transformed by a linear operator $\psi$ such that

$$
\begin{aligned}
\psi(\mathrm{y}) & =\beta_{0}^{\prime}+\psi\left(\beta_{1} \mathrm{x}_{1}\right) \ldots+\psi(\varepsilon) \\
& =\beta_{0}^{\prime}+\beta_{1} \psi\left(\mathrm{x}_{1}\right) \ldots+\psi(\varepsilon)
\end{aligned}
$$

What is different is the intercept term and, in general, the covariance structure of the error terms. By choosing the wavelet transform for our linear operator, we can fit scale-specific models in the sense that the transform is extracting scale-specific components of pattern at each level of the decomposition. For a more detailed description, see Keitt and Urban (2005), and Carl and Kühn (2008). 


\section{Appendix 5}

Model selection results based on minimization of Akaike's Information Criterion adjusted for small sample sizes (AICc) to identify the best resource utilization function for habitat use of American black bears in the Massif de la Jacques-Cartier, Québec, Canada. Only models having $\triangle \mathrm{AICc} \leq 2$ and weights $\geq 0.01$ are presented. (A) structural and functional landscape heterogeneity, and (B) local proportion of different vegetation age-classes.

\begin{tabular}{llrrrr}
\hline Level & Model structure & LogLik & AICc & $\Delta$ AICc & Weight \\
\hline (A) Structural and functional landscape heterogeneity & & & \\
Level 1 & FORAGE + SIDI + FORAGE×SIDI & -154011 & 308030.1 & 0 & 1 \\
Level 2 & FORAGE + SIDI + FORAGE×SIDI & -122041 & 244089.9 & 0 & 0.578 \\
& FORAGE & -122043 & 244090.6 & 0.633 & 0.421 \\
Level 3 & FORAGE + SIDI + FORAGEXSIDI & -119533 & 239074.8 & 0 & 1 \\
Level 4 & FORAGE + SIDI + FORAGE×SIDI & -124167 & 248341.4 & 0 & 1 \\
Level 5 & FORAGE + SIDI + FORAGE×SIDI & -115566 & 231139.5 & 0 & 1 \\
\hline
\end{tabular}




\begin{tabular}{|c|c|c|c|c|c|}
\hline Level & Model structure & LogLik & $\mathrm{AICC}$ & $\triangle \mathrm{AICC}$ & Weight \\
\hline \multicolumn{6}{|c|}{ (B) Local proportion of vegetation age-class } \\
\hline \multirow[t]{13}{*}{ Level 1} & $\%$ Shrub & -154016 & 308035.5 & 0 & 0.115 \\
\hline & $\%$ Mature & -154016 & 308035.6 & 0.016 & 0.114 \\
\hline & $\%$ Mature $+\%$ Young & -154015 & 308035.6 & 0.028 & 0.113 \\
\hline & $\%$ Mature + \% Shrub & -154015 & 308035.9 & 0.326 & 0.098 \\
\hline & $\begin{array}{l}\% \text { Mature }+\% \text { Young }+\% \\
\text { Regenerating }\end{array}$ & -154014 & 308036.2 & 0.649 & 0.083 \\
\hline & $\%$ Mature $+\%$ Young $+\%$ Shrub & -154014 & 308036.4 & 0.822 & 0.076 \\
\hline & Intercept only & -154017 & 308036.4 & 0.901 & 0.073 \\
\hline & $\%$ Clearcut $+\%$ Shrub & -154015 & 308036.5 & 0.981 & 0.070 \\
\hline & $\%$ Young $+\%$ Shrub & -154015 & 308036.8 & 1.258 & 0.061 \\
\hline & $\%$ Mature + \% Regenerating & -154015 & 308036.9 & 1.344 & 0.058 \\
\hline & $\%$ Young & -154017 & 308037.4 & 1.896 & 0.044 \\
\hline & $\%$ Regenerating $+\%$ Shrub & -154016 & 308037.4 & 1.900 & 0.044 \\
\hline & $\%$ Mature $+\%$ Clearcut & -154016 & 308037.5 & 1.935 & 0.043 \\
\hline \multirow[t]{5}{*}{ Level 2} & $\%$ Young $+\%$ Regenerating & -122045 & 244095.2 & 0 & 0.355 \\
\hline & $\begin{array}{l}\% \text { Young }+\% \text { Regenerating }+\% \\
\text { Clearcut }\end{array}$ & -122044 & 244096.5 & 1.342 & 0.181 \\
\hline & $\%$ Regenerating & -122046 & 244096.6 & 1.381 & 0.178 \\
\hline & $\begin{array}{l}\% \text { Mature }+\% \text { Young }+\% \\
\text { Regenerating }\end{array}$ & -122044 & 244097 & 1.796 & 0.144 \\
\hline & $\begin{array}{l}\% \text { Young }+\% \text { Regenerating }+\% \\
\text { Shrub }\end{array}$ & -122045 & 244097 & 1.850 & 0.140 \\
\hline \multirow[t]{3}{*}{ Level 3} & $\begin{array}{l}\% \text { Young }+\% \text { Regenerating }+\% \\
\text { Shrub }\end{array}$ & -119537 & 239081.1 & 0 & 0.569 \\
\hline & $\begin{array}{l}\% \text { Mature }+\% \text { Young }+\% \\
\text { Regenerating }+\% \text { Shrub }\end{array}$ & -119536 & 239083 & 1.917 & 0.218 \\
\hline & $\begin{array}{l}\% \text { Young }+\% \text { Regenerating }+\% \\
\text { Clearcut }+\% \text { Shrub }\end{array}$ & -119537 & 239083.1 & 1.976 & 0.211 \\
\hline Level 4 & $\begin{array}{l}\% \text { Mature }+\% \text { Young }+\% \\
\text { Regenerating }+\% \text { Clearcut }+\% \\
\text { Shrub }\end{array}$ & -124152 & 248315.3 & 0 & 1 \\
\hline Level 5 & $\begin{array}{l}\% \text { Mature }+\% \text { Young }+\% \\
\text { Regenerating }+\% \text { Clearcut }+\% \\
\text { Shrub }\end{array}$ & -114953 & 229917.8 & 0 & 1 \\
\hline
\end{tabular}


6 General discussion 


\subsection{Introduction}

The overarching goal of this thesis was to investigate and gain insight into the population consequences of landscape heterogeneity in patchy environments. Of the many factors that affect population dynamics and persistence, landscape heterogeneity is increasingly recognized as important, and particularly relevant to biodiversity conservation and management (Lovett et al., 2005; Wu \& Hobbs, 2007). However, studies considering the effects of landscape heterogeneity on population dynamics are few (Benton et al., 2003). Some authors have suggested that the effects of heterogeneity may be widespread, and may buffer a broad range of taxa against habitat changes (Lindenmayer et al., 2005; Dover \& Settele, 2009). Yet the detailed knowledge of the relationship between landscape heterogeneity and population dynamics in patchy habitats is very poor (Oliver et al., 2010).

This thesis brings together several chapters on how landscape heterogeneity affects population dynamics and persistence in patchy environments. In this final chapter, the most important results from the thesis are brought together to gain a better understanding of the roles of landscape heterogeneity in determining population dynamics. I first discuss the relative importance of within-patch heterogeneity and patch area and isolation in the determination of population dynamics. Second, I discuss the potential effects of spatial pattern of within-patch heterogeneity on population in fragmented landscapes. Third, the joint effects of the scale of spatial autocorrelation and species' life-history traits on population dynamics are discussed at both population and individual levels. Fourth, I discuss the differential roles of structural and functional landscape heterogeneity in shaping animal space use patterns. Finally, a general discussion of the thesis and suggestions for further research is provided.

\subsection{Relative influences of patch geometry and within-patch heterogeneity on population dynamics}

The results presented in chapter 2 show that within-patch variation in habitat quality, together with patch area, determined population dynamics of the specialist species, while the population dynamics of the generalist species was driven by patch area and isolation. In addition, increasing spatiotemporal variation in habitat quality had negative impact on population size of the specialist species, but had little effect on population size of the generalist species. These findings are generally in line with the previous studies that have noted species' traits-dependent susceptibility to habitat fragmentation (Davies et al., 2000; Henle et al., 2004; Ewers \& Didham, 
2006). For instance, habitat fragmentation can severely restrict the spread of specialists with poor dispersing capacity, thereby decreasing the probability of finding suitable territory which further decrease an individual's fitness (e.g., Fahrig, 2001; Wiegand et al., 2005). In contrast, the generalists may have a higher probability of occupying suitable territory under the same level of fragmentation, however, if individuals spend more time in unsuitable area due to habitat fragmentation, they will suffer high dispersal mortality, leading to decline in populations (e.g., Fahrig, 1998; Smith \& Hellmann, 2002). However, we found that spatial variation in within-patch habitat quality had a much stronger impact on the specialist species than the generalist species, indicating that the specialists may be susceptible to local variability within habitats. These species-specific response to fragmentation and local spatial heterogeneity highlight the need to recognize species life-history traits as an important dimension relevant to testing the effects of habitat fragmentation (Fahrig, 2003; Ewers \& Didham, 2006). In view of our results, habitat quality may be more relevant than patch connectivity in the population dynamics of habitat specialists, even for those with relatively high dispersal abilities, but the degree of association for each factor may vary under different landscape conditions. Empirical testing of the hypothesis confirmed that populations are less variable in size on heterogeneous compared with homogeneous patches. It is hard to judge the general applicability of these findings in a broader ecological and biodiversity context. Our study should be viewed, therefore, as a basis for future studies that consider simultaneously the effects of spatial and temporal variations in habitat quality on populations in fragmented landscapes.

\subsection{Impacts of spatial pattern of within-patch heterogeneity on patchy populations}

In its broadest sense, a landscape is an area of land containing a pattern that affects, and is affected by, an ecological process of interest such as herbivore foraging and migration. Such patterns, usually reflect the spatially variable distribution and abundance of vital resources, and are suggested to have important functional consequences (Lovett et al., 2005). We therefore tested the potential effects of spatial pattern of within-patch habitat quality (i.e., negatively autocorrelated, randomly distributed, and positively autocorrelated) on populations in fragmented landscapes. The results show explicitly that populations become less variable in size, and experience lower probability of extinction in landscapes with positively autocorrelated withinpatch habitat quality. Specifically, specialised species are more vulnerable to habitat fragmentation, but this vulnerability is greatly mitigated by positively autocorrelated habitat quality within patches, in other words, exhibiting higher resistance to habitat fragmentation. These findings point to the importance of spatial arrangement within habitats in the face of increasing 
habitat fragmentation. Given a limited movement capacity, individuals inhabiting landscapes with positively autocorrelated habitat quality are likely to encounter high-quality cells, hence, increasing the fitness of individuals as well as reducing the probability of mortality. In contrast, habitat quality in a regular pattern (negatively autocorrelated in space) results in substantial contrast between adjacent sites at a fine scale, thereby decreasing the probability of finding high-quality cells. For example, when within-patch habitat quality was positively autocorrelated, the mean habitat quality of cells occupied was the highest, indicating that individuals were confined to cells with higher habitat quality, especially for populations of specialised species. Negatively autocorrelated or randomly distributed within-patch habitat quality, if present, could amplify the impacts of habitat fragmentation by decreasing the probability of population recovery from local extinction. Moreover, these results reflect the limitations imposed on populations by their life-history traits interacting with habitat fragmentation and spatial pattern of habitat quality. For instance, a species with a narrow niche-width has a relative low probability of survival, and consequently, its persistence depends heavily on the chance of individuals to find suitable habitats (Fraterrigo et al., 2009). On the other hand, when a species' dispersal is limited, a metapopulation structure (Hanski, 1999) can emerge within habitat patches, where local populations are connected by infrequent long-distance dispersal.

\subsection{Joint effects of landscape spatial structure and species' life-history traits on population dynamics}

There has been little consensus about the population dynamics response to landscape spatial autocorrelation structure (Petchey, 2000). For example, Engen et al. (2002) suggested that the spatial scale at which environmental correlation occurs critically influences extinction risk because large-scale events have a greater impact than do small-scale events, whereas Gonzalez and Holt (2002) noted that environmental autocorrelation can have positive, rather than negative, effects on population size. In this thesis, we found that the effect of landscape spatial structure depends not only on the scale of spatial autocorrelation in habitat quality, but also on species' movement capacity and habitat requirements. As shown in Chapter 4, increasing scale of spatial autocorrelation in habitat quality would greatly increase the size and mean resource share of virtual populations, and low-tolerant species were appreciably greater in size than high-tolerant species. These observed patterns may be partly explained by edge effects, which is one of the factors influencing population dynamics (Ries et al., 2004). As the scale of spatial autocorrelation increase, habitat quality becomes less rugged across space, 
forming fewer but larger suitable areas, and consequently reduces changes in abundance or fitness of low-tolerant species with respect to low quality of "edge" areas (Pearson \& Fraterrigo, 2011). In contrast, highly tolerant species, due to higher tolerances to variation in habitat quality, may be insensitive to such edge effects.

Spatial autocorrelation in habitat quality can result in neighbourhoods of high-quality and low-quality areas, which could act similarly as "source" or "sink" habitats, thereby creating source-sink dynamics in relation to habitat heterogeneity (Dhondt et al., 1992; Rodenhouse et al., 1997). In this regard, species' movement capacity plays a critical role in shaping the increase in population size in response to increased spatial autocorrelation in habitat quality (Snyder \& Chesson, 2003; Pinto \& MacDougall, 2010). We found that low-mobility species had a logarithmic-like increase in response to increased scale of spatial autocorrelation, contrasting with the exponential-like increase for high-mobility species. Such contrasting patterns of population increase in relation to movement capacity indicate that the effect of distance-based movement capacity may function in highly scale-dependent ways, and its relative strength is determined by the interaction of movement distance and the scale of spatial autocorrelation (Snyder \& Chesson, 2003; Gilbert \& Lechowicz, 2004; Pinto \& MacDougall, 2010). Moreover, the interplay between spatial autocorrelation in habitat quality and species life-history traits can lead to spatial aggregation of individuals, creating variation in demographic performance among individuals (Dhondt et al., 1992; Rodenhouse et al., 1997). As reported in chapter 4, the proportions of individuals in high-quality cells and the proportion of individuals experiencing competition increased differentially in response to increased spatial autocorrelation between species with different movement capacity. Such nonlinear relationships indicate that movement capacity and environmental autocorrelation can jointly influence the outcome of competitive interactions (Nevoux et al., 2010), for instance, increasing spatial autocorrelation in habitat quality could greatly improve the quality of individuals by increasing the probability of finding high-quality habitat within the range of movement, which buffer the density-dependent effects on the population (Schiegg, 2003).

\subsection{Differential roles of structural and functional landscape heterogeneity in animal space use}

The functional relationship between habitat utilization and landscape heterogeneity is fundamental to understanding and predicting animal distribution (Wiens, 1989; Kolasa \& Rollo, 1991). An explicit separation of the effects of landscape heterogeneity across scales would improve our understanding of the spatial nature of animal-environment relationships. In 
this thesis, we combined wavelet transform with mixed-effects resource utilization functions (RUFs) to extract scale-specific relationships between different forms of spatial heterogeneity and habitat use by American black bears within their home ranges. Our analysis demonstrates that the waveletbased method provides an efficient way of analysing scale-specific patterns of animal space use in relation to spatial variations of landscape attributes. We found strong differences in the effects of structural and functional spatial heterogeneity and the scales at which they are associated with the patterns of habitat use by black bears. For examples, FORAGE (functional heterogeneity) were found to have significant effects at scales $800-\mathrm{m}$ and 1600-m, whereas SIDI (structural heterogeneity) alone did not have significant influences on utilization distributions of black bears at all scales investigated. Such differences in the characteristic scales of the black bears' response to structural and functional heterogeneity suggest that the spatial scaling of animal-habitat relationships should consider not only the structural and functional properties of landscapes, but also the variation in scaledependent responses of animals to the functional heterogeneity in the landscape. We also found that black bears avoided mature conifer forest at broad scales, concentrating on regenerating forest and shrubland at local scales. These results extended previous findings of Brodeur et al. (2008) and indicate that post-logging forest stands with high fruit density were important in shaping bears' habitat use at a variety of scales (Welch et al., 1997). Distinguishing functional heterogeneity from structural heterogeneity can help improve the interpretations of scale-dependent species-habitat associations (Lawler \& Edwards, 2006), and to identify threats for species and prescribing management actions, especially for those in human-altered landscapes (Wiens, 1989).

Besides being able to characterise the spatial signatures of different forms of landscape heterogeneity and their effects, our analyses showed that there were notable differences between the sexes in scale-specific use of particular resources within the home range. For instance, space use of males was highly correlated with the proportion of shrublands at a broad scale, whereas space use of females was associated with the local proportions of young forests and regenerating forests at intermediate scales. This is likely due to the sexual differences in body mass, because female bears (normally with low body mass) have lower metabolic costs and consequently have a higher energetic efficiency in less productive habitats (e.g. young forests) or smaller home range size (Welch et al., 1997). This allows a small individual to gain a higher proportion of its body mass as fat reserves with fewer resources. Furthermore, the proportion of mature forests also influenced fine scale space use of females, differing from earlier work in this region (e.g., Brodeur et al., 2008), perhaps because mature forest cover is essential to shelter cubs and escape danger (Samson \& Huot, 1998; White et al., 2001). Our 
results suggest that sex variability should be considered to make ecological inference about population-level response to habitat alterations (Lawler \& Edwards, 2006; Bastille-Rousseau et al., 2011). In addition, the mixed resource utilization function (mixed-RUF) analyses also confirm that more generalized species are more ecologically heterogeneous in their behaviours (Bolnick et al., 2007), opening research into the consequences of individual/group heterogeneity to population dynamics which has important implications for biodiversity conservation and management.

\subsection{General conclusion}

This thesis highlights the importance of the landscape heterogeneity in determining the dynamics and persistence of populations inhabiting patchy environments. Both the spatial pattern and extent of landscape heterogeneity can interact with fragmentation to greatly influence populations with different life-history traits. Ecologists and natural resource managers need to be mindful of the simplifying assumptions of theories and resulting models and predictions in assessing the suitability of their application for specific species and landscapes. Further, structural and functional properties of landscape heterogeneity, which can have different consequences on animal species, should be explicitly considered to improve our understanding of the spatial nature of animal-environment relationships.

\subsection{Suggestions for future research}

Despite the wide-spread recognition and application of the patch-matrix paradigm, this study suggests that a more inclusive conceptual framework is required to integrate the influence of landscape spatial and temporal heterogeneity on population dynamics and persistence in landscapes of variable quality. First at the theoretical investigation level, the models in this thesis were simplified in order to generalize population response to landscape heterogeneity and fragmentation. For example, the consequence of different movement modes on populations was not examined in the model despite recent research suggesting that non-random movement may promote the persistence of populations in fragmented landscapes (e.g., Thomas, 2000; Johst et al., 2002; Reed, 2004). Further research is required to test the extent of spatial abilities in individuals and to determine if some individuals are more adept than others in heterogeneous environments.

Second, the exogenous and endogenous threats should be considered in future simulation investigation. Exogenous stochastic threats are related to environmental variability, such as fluctuations in climate or natural catastrophes, while endogenous stochastic threats occur as part of a species' 
life cycle, including demographic and genetic stochasticity, which are more pronounced in small populations (Keller \& Waller, 2002).

Third, the results from our simulations are contingent upon numerous model assumptions, and it remains to be seen whether the same or similar effects also occur for species living in real landscapes. Recent advances in navigational technology will provide new and improved ways to accurately track animal movements and behaviour. For example, global positioning system (GPS) receivers have been incorporated into collars and placed on animals. Data collected from GPS units might be used to test how environmental heterogeneity affects spatial movement, space use, and distribution patterns of animals across landscapes.

Fourth, the specific conditions under which landscape heterogeneity effects are expected to be the most acute, are currently unclear, and therefore require a more comprehensive and realistic approach to measuring and representing heterogeneous landscapes. Future research could investigate larger spatial and temporal scales, for example through the use of welldesigned natural experiments. 


\section{Bibliography}

Andrén, H. (1994) Effects of habitat fragmentation on birds and mammals in landscapes with different proportions of suitable habitat : a review. Oikos, 71, 355-366.

Armsworth, P. (2009) Conditional dispersal, clines, and the evolution of dispersiveness. Theoretical Ecology, 2, 105-117.

Bakeman, R. (2005) Recommended effect size statistics for repeated measures designs. Behavior Research Methods and Instrumentation, 37, 379-384.

Bastille-Rousseau, G., Fortin, D., Dussault, C., Courtois, R. \& Ouellet, J.-P. (2011) Foraging strategies by omnivores: are black bears actively searching for ungulate neonates or are they simply opportunistic predators? Ecography, 34, 588-596.

Bates, D. \& Maechler, M. (2009) Ime4: Linear mixed-effects models using S4 classes. $R$ package version 0.999999-2. http://CRAN.Rproject.org/package $=$ lme4.

Benton, T.G., Vickery, J.A. \& Wilson, J.D. (2003) Farmland biodiversity: is habitat heterogeneity the key? Trends in Ecology \& Evolution, 18, 182188.

Berec, L. (2002) Techniques of spatially explicit individual-based models: construction, simulation, and mean-field analysis. Ecological Modelling, 150, 55-81.

Boeye, J., Kubisch, A. \& Bonte, D. (2014) Habitat structure mediates spatial segregation and therefore coexistence. Landscape Ecology, 29, 593604.

Boileau, F., Crête, M. \& Huot, J. (1994) Food habits of the black bear, Ursus americanus, and habitat use in Gaspésie Park, eastern Québec. Canadian Field-Naturalist, 108, 162-169.

Bolker, B.M. (2003) Combining endogenous and exogenous spatial variability in analytical population models. Theoretical Population Biology, 64, 255-270.

Bolnick, D.I., Svanbäck, R., Araújo, M.S. \& Persson, L. (2007) Comparative support for the niche variation hypothesis that more generalized populations also are more heterogeneous. Proceedings of the National Academy of Sciences, 104, 10075-10079.

Bowler, D.E. \& Benton, T.G. (2005) Causes and consequences of animal dispersal strategies: relating individual behaviour to spatial dynamics. Biological Reviews, 80, 205-225.

Box, G., Hunter, S. \& Hunter, W. (2005) Statistics for experimenters: design, innovation, and discovery. Wiley-Interscience.

Boyce, M.S. (2006) Scale for resource selection functions. Diversity and Distributions, 12, 269-276. 
Boyce, M.S., Mao, J.S., Merrill, E.H., Fortin, D., Turner, M.G., Fryxell, J. \& Turchin, P. (2003) Scale and heterogeneity in habitat selection by elk in Yellowstone National Park. Ecoscience, 10, 421-431.

Brachet, S., Olivieri, I., Godelle, B., Klein, E., Frascaria-Lacoste, N. \& Gouyon, P.-H. (1999) Dispersal and metapopulation viability in a heterogeneous landscape. Journal of Theoretical Biology, 198, 479495.

Bradshaw, G.A. \& Spies, T.A. (1992) Characterizing canopy gap structure in forests using wavelet analysis. Journal of Ecology, 80, 205-215.

Brodeur, V., Ouellet, J.-P., Courtois, R. \& Fortin, D. (2008) Habitat selection by black bears in an intensively logged boreal forest. Canadian Journal of Zoology, 86, 1307-1316.

Bruce, A. \& Gao, H.Y. (1996) Applied Wavelet Analysis with S-Plus. SpringerVerlag New York, Inc.

Burgess, M.D., Nicoll, M.A., Jones, C.G. \& Norris, K. (2011) Multiple environmental gradients affect spatial variation in the productivity of a tropical bird population. Journal of Animal Ecology, 80, 688-95.

Burnham, K.P. \& Anderson, D.R. (2002) Model selection and multimodel inference: a practical information-theoretic approach, $2^{\text {nd }}$ edn. Springer, New York.

Cardillo, M., Mace, G.M., Jones, K.E., Bielby, J., Bininda-Emonds, O.R.P., Sechrest, W., Orme, C.D.L. \& Purvis, A. (2005) Multiple causes of high extinction risk in large mammal species. Science, 309, 1239-1241.

Carl, G. \& Kühn, I. (2008) Analyzing spatial ecological data using linear regression and wavelet analysis. Stochastic Environmental Research and Risk Assessment 22, 315-324.

Cazelles, B., Chavez, M., Berteaux, D., Ménard, F., Vik, J., Jenouvrier, S. \& Stenseth, N. (2008) Wavelet analysis of ecological time series. Oecologia, 156, 287-304.

Chipperfield, J.D., Dytham, C. \& Hovestadt, T. (2011) An Updated Algorithm for the Generation of Neutral Landscapes by Spectral Synthesis. PLoS $O N E, 6$, e17040.

Clarke, R.T., Thomas, J.A., Elmes, G.W. \& Hochberg, M.E. (1997) The effects of spatial patterns in habitat quality on community dynamics within a site. Proceedings of the Royal Society of London B Biological Sciences, 264, 347-354.

Clavel, J., Julliard, R. \& Devictor, V. (2010) Worldwide decline of specialist species: toward a global functional homogenization? Frontiers in Ecology and the Environment, 9, 222-228.

Cohen, J. (1988) Statistical power analysis for the behavioral sciences, $2^{\text {nd }}$ edn. Lawrence Erlbaum Associates, Hillsdale, NJ.

Csillag, F. \& Kabos, S. (2002) Wavelets, boundaries, and the spatial analysis of landscape pattern. Ecoscience, 9, 177-190. 
Dal Forno, A. \& Merlone, U. (2004) Personnel turnover in organizations: an agent-based simulation model. Nonlinear Dynamics Psychol Life Sci, 8, 205-30.

Dale, M.R.T. \& Mah, M. (1998) The use of wavelets for spatial pattern analysis in ecology. Journal of Vegetation Science, 9, 805-814.

Daubechies, I. (1992) Ten lectures on wavelets. Society for industrial and applied mathematics, Philadelphia, Pennsylvania, USA.

Davies, K.F., Margules, C.R. \& Lawrence, K.F. (2000) Which traits of species predict population declines in experimental forest fragments? Ecology, 81, 1450-1461.

de Knegt, H.J., van Langevelde, F., Coughenour, M.B., Skidmore, A.K., de Boer, W.F., Heitkönig, I.M.A., Knox, N.M., Slotow, R., van der Waal, C. \& Prins, H.H.T. (2010) Spatial autocorrelation and the scaling of species-environment relationships. Ecology, 91, 2455-2465.

DeAngelis, D.L. \& Mooij, W.M. (2005) Individual-based modeling of ecological and evolutionary processes. Annual Review of Ecology, Evolution, and Systematics, 147-168.

Denoel, M. \& Lehmann, A. (2006) Multi-scale effect of landscape processes and habitat quality on newt abundance: Implications for conservation. Biological Conservation, 130, 495-504.

Devictor, V., Julliard, R. \& Jiguet, F. (2008) Distribution of specialist and generalist species along spatial gradients of habitat disturbance and fragmentation. Oikos, 0, 080211051304426-0.

Dhondt, A.A., Kempenaers, B. \& Adriaensen, F. (1992) Density-dependent clutch size caused by habitat heterogeneity. Journal of Animal Ecology, 61, 643-648.

Diffendorfer, J.E., Gaines, M.S. \& Holt, R.D. (1995) Habitat fragmentation and movements of 3 small mammals (Sigmodon, Microtus, and Peromyscus). Ecology, 76, 827-839.

Donner, D.M., Ribic, C.A. \& Probst, J.R. (2010) Patch dynamics and the timing of colonization-abandonment events by male Kirtland's Warblers in an early succession habitat. Biological Conservation Restoration and Sustainability, 143, 1159-1167.

Dormann, C.F., McPherson, J.M., Araújo, M.B., Bivand, R., Bolliger, J., Carl, G., Davies, R.G., Hirzel, A., Jetz, W. \& Kissling, W.D. (2007) Methods to account for spatial autocorrelation in the analysis of species distributional data: a review. Ecography, 30, 609Á628.

Dover, J. \& Settele, J. (2009) The influences of landscape structure on butterfly distribution and movement: a review. Journal of Insect Conservation, 13, 3-27.

Dussault, C., Courtois, R., Ouellet, J.-P. \& Huot, J. (2001) Influence of satellite geometry and differential correction on GPS location accuracy. Wildlife Society Bulletin, 29, 171-179. 
Dutilleul, P. \& Legendre, P. (1993) Spatial heterogeneity against heteroscedasticity: an ecological paradigm versus a statistical concept. Oikos, 66, 152-171.

Engen, S., Lande, R. \& Saether, B.E. (2002) The spatial scale of population fluctuations and quasi-extinction risk. Am Nat, 160, 439-51.

Ewers, R.M. \& Didham, R.K. (2006) Confounding factors in the detection of species responses to habitat fragmentation. Biological Reviews of the Cambridge Philosophical Society, 81, 117-42.

Fahrig, L. (1997) Relative effects of habitat loss and fragmentation on population extinction. Journal of Wildlife Management, 61, 603-610.

Fahrig, L. (1998) When does fragmentation of breeding habitat affect population survival? Ecological Modelling, 105, 273-292.

Fahrig, L. (2001) How much habitat is enough? Biological Conservation, 100 , 65-74.

Fahrig, L. (2002) Effect of habitat fragmentation on the extinction threshold: a synthesis. Ecological Applications, 12, 346-353.

Fahrig, L. (2003) Effects of habitat fragmentation on biodiversity. Annual review of ecology, evolution, and systematics, 487-515.

Fahrig, L. (2007) Non-optimal animal movement in human-altered landscapes. Functional Ecology, 21, 1003-1015.

Fahrig, L., Baudry, J., Brotons, L., Burel, F.G., Crist, T.O., Fuller, R.J., Sirami, C., Siriwardena, G.M. \& Martin, J.L. (2011) Functional landscape heterogeneity and animal biodiversity in agricultural landscapes. Ecology Letters, 14, 101-12.

Finlayson, G.R., Vieira, E.M., Priddel, D., Wheeler, R., Bentley, J. \& Dickman, C.R. (2008) Multi-scale patterns of habitat use by re-introduced mammals: a case study using medium-sized marsupials. Biological Conservation, 141, 320-331.

Fischer, J. \& Lindenmayer, D.B. (2007) Landscape modification and habitat fragmentation: a synthesis. Global Ecology and Biogeography, 16, 265-280.

Flather, C.H. \& Bevers, M. (2002) Patchy reaction-diffusion and population abundance: the relative importance of habitat amount and arrangement. The American Naturalist, 159, 40-56.

Fleishman, E., Ray, C., Sjögren-Gulve, P., Boggs, C.L. \& Murphy, D.D. (2002) Assessing the roles of patch quality, area, and isolation in predicting metapopulation dynamics. Conservation Biology, 16, 706-716.

Franken, R.J. \& Hik, D.S. (2004) Influence of habitat quality, patch size and connectivity on colonization and extinction dynamics of collared pikas ochotona collaris. Journal of Animal Ecology, 73, 889-896.

Franzén, M. \& Nilsson, S.G. (2010) Both population size and patch quality affect local extinctions and colonizations. Proceedings of the Royal Society B: Biological Sciences, 277, 79-85. 
Fraterrigo, J.M., Pearson, S.M. \& Turner, M.G. (2009) Joint effects of habitat configuration and temporal stochasticity on population dynamics. Landscape Ecology, 24, 863-877.

Fryxell, J.M., Wilmshurst, J.F., Sinclair, A.R.E., Haydon, D.T., Holt, R.D. \& Abrams, P.A. (2005) Landscape scale, heterogeneity, and the viability of Serengeti grazers. Ecology Letters, 8, 328-335.

Fujiwara, M. (2009) Environmental Stochasticity. In: Encyclopedia of Life Sciences. John Wiley \& Sons, Ltd, Chichester.

Getz, W.M. \& Saltz, D. (2008) A framework for generating and analyzing movement paths on ecological landscapes. Proceedings of the National Academy of Sciences, 105, 19066-19071.

Gilbert, B. \& Lechowicz, M.J. (2004) Neutrality, niches, and dispersal in a temperate forest understory. Proceedings of the National Academy of Sciences of the United States of America, 101, 7651-7656.

Gillies, C., Hebblewhite, M., Nielsen, S.E., Krawchuk, M., Aldridge, C., Frair, J., Stevens, C., Saher, D.J. \& Jerde, C. (2006) Application of random effects to the study of resource selection by animals. Journal of Animal Ecology, 75, 887-898.

González-Megías, A., Gómez, J.M. \& Sánchez-Piñero, F. (2005) Consequences of spatial autocorrelation for the analysis of metapopulation dynamics. Ecology, 86, 3264-3271.

Gonzalez, A. \& Holt, R.D. (2002) The inflationary effects of environmental fluctuations in source-sink systems. Proceedings of the National Academy of Sciences, 99, 14872-14877.

Grimm, V. \& Railsback, S.F. (2005) Individual-based Modeling and Ecology:(Princeton Series in Theoretical and Computational Biology).

Grimm, V., Berger, U., DeAngelis, D.L., Polhill, J.G., Giske, J. \& Railsback, S.F. (2010) The ODD protocol: A review and first update. Ecological Modelling, 221, 2760-2768.

Grimm, V., Berger, U., Bastiansen, F., Eliassen, S., Ginot, V., Giske, J., GossCustard, J., Grand, T., Heinz, S.K., Huse, G., Huth, A., Jepsen, J.U., Jørgensen, C., Mooij, W.M., Müller, B., Pe'er, G., Piou, C., Railsback, S.F., Robbins, A.M., Robbins, M.M., Rossmanith, E., Rüger, N., Strand, E., Souissi, S., Stillman, R.A., Vabø, R., Visser, U. \& DeAngelis, D.L. (2006a) A standard protocol for describing individual-based and agentbased models. Ecological Modelling, 198, 115-126.

Grimm, V., Berger, U., Bastiansen, F., Eliassen, S., Ginot, V., Giske, J., GossCustard, J., Grand, T., Heinz, S.K., Huse, G., Huth, A., Jepsen, J.U., Jorgensen, C., Mooij, W.M., Muller, B., Pe'er, G., Piou, C., Railsback, S.F., Robbins, A.M., Robbins, M.M., Rossmanith, E., Ruger, N., Strand, E., Souissi, S., Stillman, R.A., Vabo, R., Visser, U. \& DeAngelis, D.L. (2006b) A standard protocol for describing individual-based and agentbased models. Ecological Modelling, 198, 115-126. 
Guisan, A., Lehmann, A., Ferrier, S., Austin, M., Overton, J., Aspinall, R. \& Hastie, T. (2006) Making better biogeographical predictions of species' distributions. Journal of Applied Ecology, 43, 386 - 392.

Gurarie, E., Andrews, R.D. \& Laidre, K.L. (2009) A novel method for identifying behavioural changes in animal movement data. Ecology Letters, 12, 395-408.

Haefner, J.W., Poole, G.C., Dunn, P.V. \& Decker, R.T. (1991) Edge effects in computer models of spatial contagion. Ecological Modelling, 56, 221244.

Hanski, I. (1991) Single-species metapopulation dynamics: concepts, models and observations. Biological Journal of the Linnean Society, 42, 17-38.

Hanski, I. (1998) Metapopulation dynamics. Nature, 396, 41-49.

Hanski, I. (1999) Metapopulation ecology. Oxford University Press, New York.

Hanski, I. \& Gaggiotti, O. (2004) Ecology, genetics and evolution of metapopulations. Elsevier Academic Press, Amsterdam, The Netherlands.

Hanski, I., :.Pakkala, T., Kuussaari, M. \& Lei, G. (1995) Metapopulation persistence of an endangered butterfly in a fragmented landscape. Oikos 72, 21-28.

Harrison, S. \& Bruna, E. (1999) Habitat fragmentation and large-scale conservation: what do we know for sure? Ecography, 22, 225-232.

Hassell, M.P. (1975) Density Dependence in Single Species Populations. Journal of Animal Ecology, 44, 283-295.

Heaney, L.R. (2000) Dynamic disequilibrium: a long-term, largescale perspective on the equilibrium model of island biogeography. Global Ecology and Biogeography, 9, 59-74.

Heinz, S. \& Strand, E. (2006) Adaptive patch searching strategies in fragmented landscapes. Evolutionary Ecology, 20, 113-130.

Henle, K., Davies, K.F., Kleyer, M., Margules, C. \& Settele, J. (2004) Predictors of species sensitivity to fragmentation. Biodiversity and Conservation, 13, 207-251.

Hiebeler, D. (2004) Competition between near and far dispersers in spatially structured habitats. Theoretical Population Biology, 66, 205-218.

Horne, J.S., Garton, E.O., Krone, S.M. \& Lewis, J.S. (2007) Analyzing animal movements using Brownian bridges. Ecology, 88, 2354-63.

Humphries, N.E., Weimerskirch, H. \& Sims, D.W. (2013) A new approach for objective identification of turns and steps in organism movement data relevant to random walk modelling. Methods in Ecology and Evolution, 4, 930-938.

Itakura, J., Kurosaki, M., Itakura, Y., Maekawa, S., Asahina, Y., Izumi, N. \& Enomoto, N. (2010) Reproducibility and usability of chronic virus infection model using agent-based simulation; comparing with a mathematical model. Biosystems, 99, 70-8. 
Jacobson, B. \& Peres-Neto, P. (2010) Quantifying and disentangling dispersal in metacommunities: how close have we come? How far is there to go? Landscape Ecology, 25, 495-507.

Jager, H.I. (2001) Individual variation in life history characteristics can influence extinction risk. Ecological Modelling, 144, 61-76.

James, P.M., Sturtevant, B.R., Townsend, P., Wolter, P. \& Fortin, M.J. (2011) Two-dimensional wavelet analysis of spruce budworm host basal area in the Border Lakes landscape. Ecological Applications, 21, 2197-209.

Johst, K. \& Drechsler, M. (2003) Are spatially correlated or uncorrelated disturbance regimes better for the survival of species? Oikos, 103, 449-456.

Johst, K., Roland, B. \& Eber, S. (2002) Metapopulation persistence in dynamic landscapes: the role of dispersal distance. Oikos, 98, 263270.

Jonsen, I.D. \& Fahrig, L. (1997) Response of generalist and specialist insect herbivores to landscape spatial structure. Landscape Ecology, 12, 185197.

Jonsen, I.D., Flemming, J.M. \& Myers, R.A. (2005) Robust state-space modeling of animal movement data. Ecology, 86, 2874-2880.

Kadmon, R. \& Allouche, O. (2007) Integrating the effects of area, isolation, and habitat heterogeneity on species diversity: a unification of island biogeography and niche theory. The American Naturalist, 170, 443454.

Kawecki, T.J. (1995) Demography of source-sink populations and the evolution of ecological niches. Evolutionary Ecology, 9, 38-44.

Keitt, T.H. \& Urban, D.L. (2005) Scale-specific inference using wavelets. Ecology, 86, 2497-2504.

Keller, L.F. \& Waller, D.M. (2002) Inbreeding effects in wild populations. Trends in Ecology \& Evolution, 17, 230-241.

Kendall, B.E. \& Fox, G.A. (1998) Spatial structure, environmental heterogeneity, and population dynamics: analysis of the coupled logistic map. Theoretical Population Biology, 54, 11-37.

Keymer, J.E., Marquet, P.A., Velasco - Hernández, J.X. \& Simon, A.L. (2000) Extinction thresholds and metapopulation persistence in dynamic landscapes. The American Naturalist, 156, 478-494.

Kindvall, O. (1996) Habitat heterogeneity and survival in a bush cricket metapopulation. Ecology, 77, 207-214.

Klok, C. \& De Roos, A.M. (1998) Effects of habitat size and quality on equilibrium density and extinction time of Sorex araneus populations. Journal of Animal Ecology, 67, 195-209.

Kolasa, J. \& Rollo, C.D. (1991) Introduction: the heterogeneity of heterogeneity: a glossary. Ecological heterogeneity, pp. 1-23. Springer. 
Kolasa, J. \& Li, B.-L. (2003) Removing the confounding effect of habitat specialization reveals the stabilizing contribution of diversity to species variability. Proceedings of the Royal Society of London B Biological Sciences, 270, S198-S201.

Kranstauber, B. \& Smolla, M. (2013) move: Visualizing and analyzing animal track data. $R$ package version 1.1.392. http://CRAN.Rproject.org/package=move.

Kranstauber, B., Kays, R., LaPoint, S.D., Wikelski, M. \& Safi, K. (2012) A dynamic Brownian bridge movement model to estimate utilization distributions for heterogeneous animal movement. Journal of Animal Ecology, 81, 738-746.

Lawler, J.J. \& Edwards, T.C. (2006) A variance-decomposition approach to investigating multiscale habitat associations. Condor 108, 47-58.

Lawler, J.J., Aukema, J.E., Grant, J.B., Halpern, B.S., Kareiva, P., Nelson, C.R., Ohleth, K., Olden, J.D., Schlaepfer, M.A., Silliman, B.R. \& Zaradic, P. (2006) Conservation science: a 20-year report card. Frontiers in Ecology and the Environment, 4, 473-480.

Lawrence, M. (2011) ez: Easy analysis and visualization of factorial experiments. $R$ package version 3.0-0. http://CRAN.Rproject.org/package $=e z$.

Legendre, P. (1993) Spatial autocorrelation: trouble or new paradigm? Ecology, 74, 1659-1673.

Levin, S.A. (2009) The princeton guide to ecology. Princeton University Press, Princeton, NJ.

Lewis, J.S., Rachlow, J.L., Garton, E.O. \& Vierling, L.A. (2007) Effects of habitat on GPS collar performance: using data screening to reduce location error. Journal of Applied Ecology, 44, 663-671.

Lima, S.L. \& Zollner, P.A. (1996) Towards a behavioral ecology of ecological landscapes. Trends in Ecology \& Evolution, 11, 131-135.

Lindenmayer, D., Fischer, J. \& Cunningham, R. (2005) Native vegetation cover thresholds associated with species responses. Biological Conservation, 124, 311-316.

Lloyd, H.U.W. (2008) Influence of within-patch habitat quality on highAndean Polylepis bird abundance. Ibis, 150, 735-745.

Lou, Y. (2006) On the effects of migration and spatial heterogeneity on single and multiple species. Journal of Differential Equations, 223, 400-426.

Lovett, G.M., Jones, C.G., Turner, M.G. \& Weathers, K.C. (2005) Ecosystem function in heterogeneous landscapes. Ecosystem function in heterogeneous landscapes (ed. by G.M. Lovett, M.G. Turner, C.G. Jones and K.C. Weathers), pp. 1-4. Springer New York.

Manly, B.F.J., McDonald, L.L., Thomas, D.L., McDonald, T.L. \& Erickson, W.P. (2002) Resource selection by animals: statistical design and analysis for field studies, 2nd edn edn. Kluwer Academic, Dordrecht. 
Marzluff, J.M., Millspaugh, J.J. \& Handock, M.S. (2004) Relating resources to a probabilistic measure of space use: forest fragments and Stellar's jays. Ecology, 85, 1411-1427.

Matlack, G.R. \& Leu, N.A. (2007) Persistence of dispersal-limited species in structured dynamic landscapes. Ecosystems, 10, 1287-1298.

McGarigal, K. \& Marks, B.J. (1995) Fragstats: spatial pattern analysis program for quantifying landscape structure.U. S. Forest Service General Technical Report. In, Portland, OR, USA.

McKinney, M.L. (1997) Extinction vulnerability and selectivity: combining ecological and paleontological views. Annual Review of Ecology and Systematics, 28, 495-516.

Melbourne, B.A. \& Hastings, A. (2008) Extinction risk depends strongly on factors contributing to stochasticity. Nature, 454, 100-103.

Moilanen, A., Smith, A.T. \& Hanski, I. (1998) Long-term dynamics in a metapopulation of the American pika. The American Naturalist, 152, 530-542.

Morales, J.M., Haydon, D.T., Frair, J., Holsinger, K.E. \& Fryxell, J.M. (2004) Extracting more out of relocation data: building movement models as mixtures of random walks. Ecology, 85, 2436-2445.

Mortelliti, A., Amori, G. \& Boitani, L. (2010) The role of habitat quality in fragmented landscapes: a conceptual overview and prospectus for future research. Oecologia, 163, 535-547.

Muller, G., Grebaut, P. \& Gouteux, J.P. (2004) An agent-based model of sleeping sickness: simulation trials of a forest focus in southern Cameroon. C R Biol, 327, 1-11.

Murphy, D.D., Freas, K.E. \& Weiss, S.B. (1990) An environmentmetapopulation approach to population viability analysis for a threatened invertebrate. Conservation Biology, 4, 41-51.

Murwira, A. \& Skidmore, A.K. (2005) The response of elephants to the spatial heterogeneity of vegetation in a Southern African agricultural landscape. Landscape Ecology, 20, 217-234.

Nevoux, M., Gimenez, O., Arlt, D., Nicoll, M., Jones, C. \& Norris, K. (2010) Population regulation of territorial species: both site dependence and interference mechanisms matter. Proceedings of the Royal Society B: Biological Sciences,

Nieminen, M. (1996) Migration of moth species in a network of small islands. Oecologia, 108, 643-651.

Noon, B.R. \& Biles, C.M. (1990) Mathematical demography of spotted owls in the Pacific Northwest. Journal of Wildlife Management, 54, 18-27.

Olejnik, S. \& Algina, J. (2003) Generalized eta and omega squared statistics: measures of effect size for some common research designs. Psychological Methods, 8, 434-447. 
Oliver, T., Roy, D.B., Hill, J.K., Brereton, T. \& Thomas, C.D. (2010) Heterogeneous landscapes promote population stability. Ecology Letters, 13, 473-484.

Pandit, S.N., Kolasa, J. \& Cottenie, K. (2009) Contrasts between habitat generalists and specialists: an empirical extension to the basic metacommunity framework. Ecology, 90, 2253-2262.

Parrott, L. \& Kok, R. (2000) Incorporating complexity in ecosystem modelling. Complexity International, 7, Online.

Pearson, S.M. \& Fraterrigo, J.M. (2011) Habitat quality, niche breadth, temporal stochasticity, and the persistence of populations in heterogeneous landscapes. Sources, sinks and sustainability (ed. by J. Liu, V. Hull, A.T. Morzillo and J.A. Wiens). Cambridge University Press.

Pellet, J., Fleishman, E., Dobkin, D.S., Gander, A. \& Murphy, D.D. (2007) An empirical evaluation of the area and isolation paradigm of metapopulation dynamics. Biological Conservation, 136, 483-495.

Percival, D.B. \& Walden, A.T. (2000) Wavelet methods for time series analysis. Cambridge University Press, New York, USA.

Petchey, O.L. (2000) Environmental colour affects aspects of single-species population dynamics. Proceedings of the Royal Society of London. Series B: Biological Sciences, 267, 747-754.

Petchey, O.L., Gonzalez, A. \& Wilson, H.B. (1997) Effects on Population Persistence: The Interaction between Environmental Noise Colour, Intraspecific Competition and Space. Proceedings: Biological Sciences, 264, 1841-1847.

Pigliucci, M. (2001) Environmental heterogeneity: temporal and spatial. eLS. John Wiley \& Sons, Ltd.

Pike, N., Tully, T., Haccou, P. \& Ferriere, R. (2004) The effect of autocorrelation in environmental variability on the persistence of populations: an experimental test. Proceedings of the Royal Society of London. Series B: Biological Sciences, 271, 2143-2148.

Pinto, S.M. \& MacDougall, A.S. (2010) Dispersal limitation and environmental structure interact to restrict the occupation of optimal habitat. The American Naturalist, 175, 675-686.

Pittiglio, C., Skidmore, A.K., van Gils, H.A.M.J. \& Prins, H.H.T. (2013) Elephant response to spatial heterogeneity in a savanna landscape of northern Tanzania. Ecography, 36, 001-013.

Powell, R.A., Zimmerman, J.W. \& Seaman, J.W. (1997) Ecology and behaviour of North American black bears: home ranges, habitat and social organization. Chapman and Hall, New York.

Prugh, L.R., Hodges, K.E., Sinclair, A.R.E. \& Brashares, J.S. (2008) Effect of habitat area and isolation on fragmented animal populations. PNAS, 105, 20770-20775.

Pulliam, H.R. (1988) Sources, sinks, and population regulation. American Naturalist, 132, 652-661. 
Pulliam, H.R. \& Danielson, B.J. (1991) Sources, sinks, and habitat selection: a landscape perspective on population dynamics. American naturalist, S50-S66.

Pulliam, H.R., Dunning, J.B. \& Liu, J. (1992) Population dynamics in complex landscapes: a case study. Ecological Applications, 2, 165-177.

$\mathrm{R}$ Development Core Team (2011) R: A language and environment for statistical computing, version 2.13.0. $\mathrm{R}$ Foundation for Statistical Computing, Vienna, Austria.

Reding, D., Cushman, S., Gosselink, T. \& Clark, W. (2013) Linking movement behavior and fine-scale genetic structure to model landscape connectivity for bobcats (Lynx rufus). Landscape Ecology, 28, 471486.

Reed, D.H. (2004) Extinction risk in fragmented habitats. Animal Conservation, 7, 181-191.

Ricketts, T.H. (2001) The matrix matters: effective isolation in fragmented landscapes. The American Naturalist, 158, 87-99.

Ries, L., Fletcher Jr, R.J., Battin, J. \& Sisk, T.D. (2004) Ecological responses to habitat edges: mechanisms, models, and variability explained. Annual Review of Ecology, Evolution, and Systematics, 491-522.

Rodenhouse, N.L., Sherry, T.W. \& Holmes, R.T. (1997) Site-dependent regulation of population size: a new synthesis. Ecology, 78, 20252042.

Root, K.V. (1998) Evaluating the effects of habitat quality, connectivity, and catastrophes on a threatened species. Ecological Applications, 8, 854865.

Ruel, J.J. \& Ayres, M.P. (1999) Jensen's inequality predicts effects of environmental variation. Trends in Ecology \&amp; Evolution, 14, 361366.

Runge, Jonathan P., Runge, Michael C. \& Nichols, James D. (2006) The role of local populations within a landscape context: defining and classifying sources and sinks. The American Naturalist, 167, 925-938.

Samson, C. \& Huot, J. (1998) Movements of female black bears in relation to landscape vegetation in southern Quebec. Journal of Wildlife Management, 62, 718-727.

Saupe, D. (1988) Algorithms for random fractals. The Science of Fractal Images (ed. by H.O. Petigen and D. Saupe), pp. 71-113. Springer, New York.

Schick, R.S., Loarie, S.R., Colchero, F., Best, B.D., Boustany, A., Conde, D.A., Halpin, P.N., Joppa, L.N., McClellan, C.M. \& Clark, J.S. (2008) Understanding movement data and movement processes: current and emerging directions. Ecology Letters, 11, 1338-1350.

Schiegg, K. (2003) Environmental autocorrelation: curse or blessing? Trends in Ecology \& Evolution, 18, 212-214. 
Schooley, R.L. \& Branch, L.C. (2007) Spatial heterogeneity in habitat quality and cross-scale interactions in metapopulations. Ecosystems, 10, 846853.

Schooley, R.L. \& Branch, L.C. (2009) Enhancing the area-isolation paradigm: habitat heterogeneity and metapopulation dynamics of a rare wetland mammal. Ecological Applications, 19, 1708-1722.

Schreinemachers, P. \& Berger, T. (2011) An agent-based simulation model of human-environment interactions in agricultural systems. Environmental Modelling \& Software, 26, 845-859.

Shaver, G.R. (2005) Spatial heterogeneity: past, present, and future. Ecosystem Function in Heterogeneous Landscapes (ed. by G.M. Lovett, M.G. Turner, C.G. Jones and K.C. Weathers), pp. 443-449. Springer New York.

Silverman, B.W. (1986) Density estimation for statistics and data analysis. CRC press.

Skelsey, P. \& Garrett, K.A. (2013) Why dispersal should be maximized at intermediate scales of heterogeneity. Theoretical Ecology, 1-9.

Smith, J.N.M. \& Hellmann, J.J. (2002) Population persistence in fragmented landscapes. Trends in Ecology \& Evolution, 17, 397-399.

Snyder, R.E. \& Chesson, P. (2003) Local dispersal can facilitate coexistence in the presence of permanent spatial heterogeneity. Ecology Letters, 6, 301-309.

Sodhi, N.S., Brook, B.W. \& Bradshaw, C.A.J. (2009) Causes and consequences of species extinctions. Princeton Guide to Ecology (ed. by S.A. Levin), pp. 514-520. Princeton University Press, Princeton, NJ.

Stacey, P.B. \& Taper, M. (1992) Environmental variation and the persistence of small populations. Ecological Applications, 2, 18-29.

Stoddard, S.T. (2010) Continuous versus binary representations of landscape heterogeneity in spatially-explicit models of mobile populations. Ecological Modelling, 221, 2409-2414.

Stouffer, P.C. \& Bierregaard, R.O. (1995) Effects of forest fragmentation on understorey hummingbirds in Amazonian Brazil. Conservation Biology, 9, 1085-1094.

Sutcliffe, O.L., Thomas, C.D., Yates, T.J. \& Greatorex Davies, J.N. (1997) Correlated extinctions, colonizations and population fluctuations in a highly correlated ringlet butterfly metapopulation. Oecologia, 109, 235-241.

Talley, T.S. (2007) Which spatial heterogeneity framework? consequences for conclusions about patchy population distributions. Ecology, 88, 14761489.

Tang, W. \& Bennett, D.A. (2010) Agent-based modeling of animal movement: a review. Geography Compass, 4, 682-700. 
Ten Have, T.R., Kunselman, A.R. \& Tran, L. (1999) A comparison of mixed effects logistic regression models for binary response data with two nested levels of clustering. Statistics in Medicine, 18, 947-960.

Thomas, C.D. (2000) Dispersal and extinction in fragmented landscapes. Proceedings of the Royal Society B Biological Sciences, 267, 139-145.

Thomas, J.A., Bourn, N.A., Clarke, R.T., Stewart, K.E., Simcox, D.J., Pearman, G.S., Curtis, R. \& Goodger, B. (2001) The quality and isolation of habitat patches both determine where butterflies persist in fragmented landscapes. Proc. Biol. Sci., 268, 1791-1796.

Tienderen, P.H.V. (1997) Generalists, specialists, and the evolution of phenotypic plasticity in sympatric populations of distinct species. Evolution, 51, 1372-1380.

Torrence, C. \& Compo, G.P. (1998) A practical guide to wavelet analysis. Bulletin of the American Meteorological Society, 79, 61-78.

Turner, M.G. \& Chapin, F.S. (2005) Causes and consequences of spatial heterogeneity in ecosystem function. Ecosystem function in heterogeneous landscapes (ed. by G.M. Lovett, M.G. Turner, C.G. Jones and K.C. Weathers), pp. 9-30. Springer New York.

Turner, W.R. \& Tjørve, E. (2005) Scale-dependence in species-area relationships. Ecography, 28, 721-730.

Vasseur, C., Joannon, A., Aviron, S., Burel, F., Meynard, J.-M. \& Baudry, J. (2013) The cropping systems mosaic: How does the hidden heterogeneity of agricultural landscapes drive arthropod populations? Agriculture, Ecosystems \& Environment, 166, 3-14.

Villard, M.A., Trzcinski, M.K. \& Merriam, G. (1999) Fragmentation effects on forest birds: relative influence of woodland cover and configuration on landscape occupancy. Conservation biology, 13, 774-783.

Vögeli, M., Serrano, D., Pacios, F. \& Tella, J.L. (2010) The relative importance of patch habitat quality and landscape attributes on a declining steppebird metapopulation. Biological Conservation, 143, 1057-1067.

Walker, R.S., Novaro, A.J. \& Branch, L.C. (2003) Effects of patch attributes, barriers, and distance between patches on the distribution of a rockdwelling rodent (Lagidium viscacia). Landscape Ecology, 18, 185-192.

Welch, C.A., Keay, J., Kendall, C.K. \& Robbins, C.T. (1997) Constraints on frugivory by bears. Ecology, 78, 1105-1119.

Wheatley, M. \& Johnson, C. (2009) Factors limiting our understanding of ecological scale. Ecological Complexity, 6, 150-159.

Whitcher, B. (2012) waveslim: Basic wavelet routines for one-, two- and three-dimensional signal processing. $R$ package version 1.7.1. http://CRAN.R-project.org/package=waveslim.

Whitcher, B., Guttorp, P. \& Percival, D.B. (2000) Wavelet analysis of covariance with application to atmospheric time series. Journal of Geophysical Research: Atmospheres, 105, 14941-14962. 
White, J.W., Rassweiler, A., Samhouri, J.F., Stier, A.C. \& White, C. (2014) Ecologists should not use statistical significance tests to interpret simulation model results. Oikos, 123, 385-388.

White, T.H., Bowman, J., Jacobson, H.A., Leopold, B.D. \& Smith, W.P. (2001) Forest management and female black bear denning. Journal of Wildlife Management, 65, 34-40.

Wiegand, T., Revilla, E. \& Moloney, K.A. (2005) Effects of habitat loss and fragmentation on population dynamics. Conservation Biology, 19, 108121.

Wiegand, T., Moloney, K., Naves, J. \& Knauer, F. (1999) Finding the missing link between landscape structure and population dynamics: a spatially explicit perspective. The American naturalist, 154, 605-627.

Wiens, J.A. (1989) Spatial scaling in ecology. Functional Ecology, 3, 385-397.

Wiens, J.A., Stenseth, N.C., Vanhorne, B. \& Ims, R.A. (1993a) Ecological Mechanisms and Landscape Ecology. Oikos, 66, 369-380.

Wiens, J.A., Stenseth, N.C., Van Horne, B. \& Ims, R.A. (1993b) Ecological mechanisms and landscape ecology. Oikos, 66, 369-380.

Wilcox, C., Cairns, B.J. \& Possingham, H.P. (2006) The role of habitat disturbance and recovery in metapopulation persistence. Ecology, 87, 855-863.

Wilensky, U. (1999) NetLogo. http://ccl.northwestern.edu/netlogo. Center for Connected Learning and Computer-Based Modeling, Northwestern University.

Wimberly, M. (2006) Species dynamics in disturbed landscapes: when does a shifting habitat mosaic enhance connectivity? Landscape Ecology, 21, 35-46.

With, K.A. \& King, A.W. (1999) Extinction thresholds for species in fractal landscapes. Conservation Biology, 13, 314-326.

Worton, J.B. (1989) Kernel methods for estimating the utilization distribution in home range studies. Ecology, 70, 164-168.

$\mathrm{Wu}, \mathrm{J}$. (2009) Ecological dynamics in fragmented landscapes. Princeton guide to ecology (ed. by S.A. Levin), pp. 438-444. Princeton University Press, Princeton, NJ.

Wu, J., Jelinski, D.E., Luck, M. \& Tueller, P.T. (2000) Multiscale analysis of landscape heterogeneity: scale variance and pattern metrics. Geographical Information Science, 6, 6-19.

Wu, J.G. \& Hobbs, R. (2002) Key issues and research priorities in landscape ecology: An idiosyncratic synthesis. Landscape Ecology, 17, 355-365.

Wu, J.G. \& Hobbs, R.J. (2007) Key topics in landscape ecology. Cambridge University Press, Cambridge.

Ye, X., Skidmore, A.K. \& Wang, T. (2013a) Within-patch habitat quality determines the resilience of specialist species in fragmented landscapes. Landscape Ecology, 28, 135-147. 
Ye, X., Wang, T. \& Skidmore, A. (2013b) Spatial pattern of habitat quality modulates population persistence in fragmented landscapes. Ecological Research, 28, 949-958.

Zajac, T., Solarz, W. \& Bielański, W. (2008) Site-dependent population dynamics: the influence of spatial habitat heterogeneity on individual fitness in the sedge warbler Acrocephalus schoenobaenus. Journal of Avian Biology, 39, 206-214. 


\section{Summary}

Of the many factors that affect population dynamics and persistence, landscape heterogeneity is increasingly recognized as one important factor that is particularly relevant to biodiversity conservation and management. However, understanding interactions between mobile species and landscape heterogeneity remains an outstanding challenge in ecology. Studies considering the influence of landscape heterogeneity on population dynamics are less common, and detailed knowledge of the relationship between landscape heterogeneity and population dynamics in patchy habitats is very poor. This study tested how landscape heterogeneity affects population behaviour and persistence in patchy environments, including (1) relative influences of patch geometry and within-patch heterogeneity, (2) effects of spatial patterns of within-patch heterogeneity on patchy populations, (3) landscape spatial structure and species' life-history traits, and (4) differential roles of structural and functional landscape heterogeneity.

The results presented in this thesis demonstrate that spatial heterogeneity within habitat patches, together with patch area, controls population abundance of the habitat specialists, but had little influence on generalist species. Populations of specialised species become less variable in size, and experience lower probability of extinction in landscapes with positively autocorrelated within-patch habitat quality. Increasing scale of spatial autocorrelation in habitat quality would greatly increase the size and mean resource share of virtual populations, and low-tolerant species were appreciably greater in size than high-tolerant species. Species' movement capacity plays a critical role in shaping the increase in population size in response to increased spatial autocorrelation in habitat quality, where lowmobility species had a logarithmic-like increase in response to increased scale of spatial autocorrelation, contrasting with the exponential-like increase for high-mobility species. Such contrasting patterns of population increase in relation to movement capacity indicate that the effect of distance-based movement capacity may function in highly scale-dependent ways, and its relative strength is determined by the interaction of movement distance and the scale of spatial autocorrelation.

Structural and functional properties of landscape heterogeneity can have different consequences on animal species. As we shown in the thesis, functional landscape heterogeneity influenced space use of black bears at intermediate scales (i.e., $1 \sim 2 \mathrm{~km}$ ), whereas the impact of structural heterogeneity was most significant at the finest scale analysed (i.e., $200 \mathrm{~m}$ ). The scale-dependent responses of animals to different forms of landscape heterogeneity indicate that an explicit separation of the effects of different 
landscape heterogeneity across scales is critical to our understanding of the spatial nature of animal-environment relationships.

Summarizing, this study highlights the importance of the landscape heterogeneity in determining the dynamics and persistence of populations inhabiting patchy environments. Both the spatial pattern and extent of landscape heterogeneity can interact with fragmentation to greatly influence populations with different life-history traits. Ecologists need to be mindful of the simplifying assumptions of theories and resulting models and predictions in assessing the suitability of their application for specific species and landscapes. 


\section{Samenvatting}

Als het gaat om instandhouding en beheer van biodiversiteit en het management ervan, wordt heterogeniteit van het landschap als de belangrijkste factor gezien die de populatiedynamiek en persistentie beïnvloeden van dit beheer en management. Het begrijpen van de interacties tussen de mobiele soorten en de heterogeniteit van het landschap blijft echter nog steeds een behoorlijke uitdaging voor de ecology. Er zijn weinig studies bekend die de invloed van de heterogeniteit van het landschap met betrekking tot populatiedynamiek onderzoeken. Bovendien is er weinig gedetailleerde kennis op het gebied van de relatie tussen heterogeniteit van het landschap en populatiedynamiek bij gefragmenteerde landschappen. Dit onderzoek heeft getest hoe de heterogeniteit van het landschap het gedrag van de bevolking beïnvloed, alsmede de persistentie in gefragmenteerde landschappen. Hierbij is rekening gehouden met (1)de relatieve invloeden van geometrie van landschappen en de heterogeniteit binnen een stuk landschap, (2) de effecten van ruimtelijke patronen van de heterogeniteit binnen stukken landschap op de populatie, (3) de ruimtelijke structuur van het landschap en de levens-historie van de eigenschappen van bepaalde soorten en (4) de differentiële rollen van de heterogeniteit van de structuur en functionaliteit van een omgeving.

De resultaten die in dit onderzoek gepresenteerd worden laten zien dat de ruimtelijke heterogeniteit binnen het leefgebied in landschappen, tezamen met het landschaps gebied, de overvloed van bepaalde speciale soorten beïnvloeden, maar weinig invloed hebben op de algemene diersoorten. De hoeveelheid aan speciale soorten wordt minder veranderlijk en de kans op uitsterven wordt minder in landschappen met een positief auto-gecorreleerde kwaliteit. De schaalvergroting van ruimtelijke autocorrelatie qua kwaliteit van leefgebied zou de grootte en conditie van virtuele diersoorten vergroten. De grootte van de soorten die een lager tolerantie niveau hebben was hoger dan de soorten waarbij de tolerantie hoger was. De beweegruimte van de soorten speelt een kritieke rol bij het in model brengen van de toename in populatie. De minder actieve soorten vertoonden een meer logaritmische toename bij een toenemende schaal van ruimtelijke autocorrelatie. Daarentegen vertoonden de actieve (meer bewegende) soorten een exponentiële toename. Deze patronen in de toename van populatie met betrekking tot the beweegruimte laten zien dat het effect van beweegruimte in relatie tot afstand goed functioneert met betrekking tot de schaalvergroting en dat de sterkte hiervan relatief gezien wordt beïnvloed door de interactive van de afstanden die kunnen worden afgelegd en de schaal van ruimtelijke autocorrelatie. 
De structurele en functionele eigenschappen van het heterogene landschap kunnen verschillende consequenties hebben op diersoorten. Zoals het onderzoek laat zien, beïnvloedt de leefruimte in een heterogeen landschap de soort op een gemiddelde schaal (bijv: 1-2 km), terwijl de gevolgen bij een structurele heterogeniteit het meest belangrijk was op een gedetailleerde schaal (bijv: 200m). De schaal-afhankelijke reacties van dieren op de verschillende vormen van heterogene landschappen laat zien dat een expliciete scheiding tussen de effecten op verschillende schalen bij heterogene landschappen nodig is om de ruimtelijke aard tussen dieren en natuur, en de bijbehorende relaties hiertussen, beter te kunnen begrijpen.

Samenvattend benadrukt deze studie het belang van de heterogeniteit van het landschap in het bepalen van de dynamiek en het overleven van populaties die in een gefragmenteerd landschap leven. Zowel het ruimtelijke patroon en de omvang van de heterogeniteit van het landschap kan interageren op de fragmentatie en zo grote invloed hebben op de soorten met verschillende levenshistorie en eigenschappen. Ecologen moeten zich beter bewust zijn dat wanneer ze bepaalde veronderstelde theorieën proberen te vereenvoudigen, en op basis hiervan voorspellingen doen en modellen maken, deze niet de geschiktheid van de applicaties voor bepaalde soorten en landschappen in zullen kaart brengen, terwijl dit wel zou moeten. 


\section{Biography}

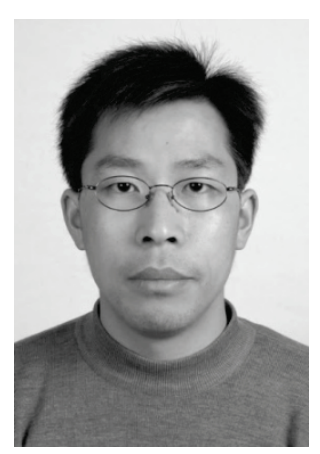

Ye, Xinping was born on 10th January 1976 in Jiangxi, China. In 1997 he obtained BSc degree in Forestry from Central South University of Forestry and Technology, China. In 2006, he received MSc degree in Zoology from Beijing Normal University, China. In the same year, he was awarded an EU scholarship for the joint MSc course on Geo-information Science and Earth Observation for Environmental Modelling and Management (GEM), and then in 2008 received MSc degree from International Institute for Geo-Information Science and Earth Observation (ITC), the Netherlands. In 2010, he received the EMECW grant from EU to pursue a PhD Degree at Faculty of Geo-Information Science and Earth Observation of the University of Twente.

He has joined Shaanxi Foping National Nature Reserve, China since 1997, and currently serves as senior forestry engineer for the reserve. His major research interests include spatial patterns in ecological data, landscape mapping, monitoring, and modelling, and biodiversity conservation. 


\section{Publications}

Ye, X., Wang, T., Skidmore, A., Fortin, D., Bastille-Rousseau, G., and Parrott, L. (2014) A wavelet-based approach to evaluate the roles of structural and functional landscape heterogeneity in animal space use at multiple scales. Ecography, 37:1-11.

Ye, X., Skidmore, A. and Wang, T. (2014) Joint effects of habitat heterogeneity and species' life-history traits on population dynamics in spatially structured landscapes. PLOS ONE, 9:e107742.

Ye, X., Skidmore, A. and Wang, T. (2013) Within-patch habitat quality determines the resilience of specialist species in fragmented landscapes. Landscape Ecology, 28:135-147.

Ye, X., Wang, T. and Skidmore, A. (2013) Spatial pattern of habitat quality modulates population persistence in fragmented landscapes. Ecological Research, 28:949-958.

Chang, J., Chen, D., Ye, X., Li, S., Liang, W., Zhang, Z. and Li, M. (2012) Coupling Genetic and Species Distribution Models to Examine the Response of the Hainan Partridge (Arborophila ardens) to Late Quaternary Climate. PLoS ONE, 7:e50286.

Wang, T., Ye, X., Skidmore, A.K. \& Toxopeus, A.G. (2010) Characterizing the spatial distribution of giant pandas (Ailuropoda melanoleuca) in fragmented forest landscapes. Journal of Biogeography, 37: 865-878.

Ye, X., Wang, T., Skidmore, A., \& Toxopeus, A.G. (2008) Characterizing distribution of giant pandas in China using MODIS data and landscape Metrics. In Proceedings of International Society for Photogrammetry and Remote Sensing, 38: 1039-1045.

Ye, X., Yong, Y., Yu, C. \& Zhang, Z. (2007) Den selection by the giant panda in Foping Nature Reserve, China. Journal of Natural History, 41: 2529-2536.

Chang, J., Ye, X., Chen, D., Li, S., Li, M. and Zhang, Z. In situ survival in response to climate change after the late Quaternary and its conservation implications: evidences from genetic data and species distribution models in the Reeves's Pheasant (Syrmaticus reevesii). Submitted to Global Change Biology. 


\section{ITC Dissertation List}

http://www.itc.nl/research/phd/phd_graduates.aspx 